\title{
DISCONTINUOUS GALERKIN APPROXIMATIONS OF THE STOKES AND NAVIER-STOKES EQUATIONS
}

\author{
KONSTANTINOS CHRYSAFINOS AND NOEL J. WALKINGTON
}

\begin{abstract}
Numerical schemes to compute approximate solutions of the evolutionary Stokes and Navier-Stokes equations are studied. The schemes are discontinuous in time and conforming in space and of arbitrarily high order. Fully-discrete error estimates are derived and dependence of the viscosity constant is carefully tracked. It is shown that the errors are bounded by projection errors of the exact solution which exhibit optimal rates when the solutions are smooth.
\end{abstract}

\section{INTRODUCTION}

We consider the evolutionary Navier-Stokes problem; that is, we seek a velocity field $\mathbf{u}$ and a pressure $p$ such that

$$
\left\{\begin{aligned}
\mathbf{u}_{t}-\nu \Delta \mathbf{u}+\mathbf{u} \cdot \nabla \mathbf{u}+\nabla p & =\mathbf{f} & & \text { in } \Omega \times(0, T], \\
\nabla \cdot \mathbf{u} & =0 & & \text { in } \Omega \times(0, T], \\
\mathbf{u}(0) & =\mathbf{u}_{0} & & \text { in } \Omega,
\end{aligned}\right.
$$

given initial data $\mathbf{u}_{0}$ and forcing term $\mathbf{f}$. Here, $\Omega$ denotes a bounded domain in $\mathbb{R}^{d}$ with $d=2,3$.

There is an abundant literature concerning analytical and computational aspects of the Stokes and Navier-Stokes equations. Various schemes based on finite elements have been proposed for approximating solutions of (1.1). However, when it comes to the analysis of higher order schemes under minimal regularity assumptions the literature is limited. In this paper we analyze a class of schemes which are conforming in space and use the discontinuous Galerkin time stepping scheme. The discontinuous Galerkin technique provides an appropriate setting to analyze adaptive refinement and coarsening strategies, and Lagrangian or moving mesh schemes, all of which give rise to different meshes at each time step. While this complicates the analysis, our results show that this does not cause any degradation in the rates of convergence.

Our estimates are expressed in terms of projections of the exact solution onto the discrete spaces which exhibit optimal rates of convergence when the standard parabolic regularity holds. Our main results are "almost symmetric" in the sense that error in the solution is essentially bounded by the projection errors in the same

Received by the editor May 16, 2009 and, in revised form, March 26, 2009.

2010 Mathematics Subject Classification. Primary 65M12, 65M60.

Key words and phrases. Navier Stokes, Discontinuous Time Stepping.

This work was supported in part by National Science Foundation Grant DMS-0811029. This work was also supported by the NSF through the Center for Nonlinear Analysis.

(C)2010 American Mathematical Society Reverts to public domain 28 years from publication 
norm. Estimates of this form show that the error will be as good as the approximation and regularity theory allows, and can be viewed as a parabolic version of the classical Cea's Lemma [10. Symmetric estimates have also been derived by Dupont, Liu and Liu, Dupont, Bank, Garcia and Santos 14, 27, to analyze moving mesh finite element methods, and by the authors in [7, 8, for a general class of implicit parabolic PDEs with time-dependent non-selfadjoint coefficients whose solutions may exhibit low regularity.

A central idea of our analysis is the development of a parabolic analog of the elliptic projection [33]. Stability of this projection does not require any relationship between the time and space partitions. The dependence of the constants appearing in the projection estimates upon the viscosity $\nu$ is carefully tracked.

1.1. Related results. Second-order semi-discrete (in space) finite element error estimates for the evolutionary Navier-Stokes equations were studied by Heywood and Rannacher in [18, and semi-discrete estimates of arbitrary order were developed by the same authors in 19 under various regularity assumptions on the solution $(\mathbf{u}, p)$. More recently, high order error estimates for the spacial discretization of the time-dependent Navier-Stokes equations where developed by Bause [4, while Hou 21] obtained symmetric error estimates of arbitrary order under minimal regularity assumptions for the evolutionary Stokes problem. High order fully-discrete schemes for smooth solutions were studied by Baker, Dougalis and Karakashian in [2], and Heywood and Rannacher [20] established second-order (in time) estimates using the Crank-Nickolson time stepping scheme under weaker regularity assumptions. For some classical results regarding the stability of various fully-discrete Galerkin schemes one may consult the book of Temam [31] or the papers by Pironeau [28] and Süli in [30] who analyzed characteristic Galerkin time stepping schemes. Finally, an analysis of an implicit Runge-Kutta nonconforming scheme was provided by Katsaounis in [24].

The discontinuous Galerkin method was first introduced to model and simulate neutron transport by Lasaint and Raviart in 25. Various aspects concerning applications of the DG scheme for hyperbolic problems were presented by Cockburn, Karnadiakis and Shu in [11] and by Johnson in 23] (see also the references within). Estimates for discontinuous solutions for the DG schemes for the transport equation were derived by Walkington in [36. The DG method for ordinary differential equations was first analyzed by Delfour, Hager and Trochu in [13] who showed that the DG scheme was superconvergent at the partition points (order $2 k+2$ for polynomials of degree $k$ ). Jamet [22] established rates of order $\mathcal{O}\left(\tau^{k}\right)$ for linear parabolic problems and subsequently Eriksson, Johnson and Thomée [15] established $\mathcal{O}\left(\tau^{2 k-1}\right)$ estimates at the partition points for smooth solutions. An excellent exposition of their results and, more generally, the DG method for parabolic equations, can be found in Thomée's book [33. Recently there has been a lot of work on the development and analysis of discontinuous Galerkin methods for elliptic problems. A comprehensive survey and comparison of this work was given by Arnold, Brezzi, Cockburn and Marini in [1] which contains many references related to this approach.

1.2. Outline. The standard abstract setting for the Navier-Stokes equations and the numerical schemes we consider, are introduced in the next section. One of the limitations of DG time stepping schemes is that the discrete energy estimate only 
bounds the solution at the partition points $\left\{t^{n}\right\}$. In order to bound the solution at times between these, fine properties of polynomials are needed. These are developed in Section 3 ,

To circumvent the technical difficulties associated with the discontinuities introduced at each time step we introduce a parabolic analog of the classical elliptic projection 33. This parabolic projection is developed in Section 4 and, like its elliptic counterpart, is the discrete solution of an associated linear (Stokes) problem. Error estimates for the Navier-Stokes equations are then developed in Section 5.

1.3. Notation. It will be assumed that the equations are to be solved in a bounded connected Lipschitz domain $\Omega \subset \mathbb{R}^{d}$ with $d=2$ or $d=3$. Vector-valued functions on $\Omega$ will be denoted with bold face, $\mathbf{u}, \mathbf{v}, \mathbf{w}$. Standard notation is used for Sobolev spaces of scalar and vector-valued functions, with norms denoted $\|\cdot\|_{H^{s}(\Omega)}$. We also denote solenoidal (divergence free) spaces by $\mathcal{V}(\Omega), V(\Omega)$ and $W(\Omega)$ where,

$$
\begin{gathered}
\mathcal{V}(\Omega)=\left\{u \in C_{0}^{\infty}(\Omega): \nabla \cdot \mathbf{u}=0\right\}, \\
V(\Omega)=\left\{u \in H_{0}^{1}(\Omega): \nabla \cdot \mathbf{u}=0\right\}, \\
W(\Omega)=\left\{u \in L^{2}(\Omega): \nabla \cdot \mathbf{u}=0 \text { and } \mathbf{u} \cdot \mathbf{n}=0\right\} .
\end{gathered}
$$

The spaces $V(\Omega)$ and $W(\Omega)$ are equipped with the $H^{1}(\Omega)$ and $L^{2}(\Omega)$ norms respectively. With $\Omega$ as above, $V(\Omega)$ and $W(\Omega)$ are closed subspaces of $H_{0}^{1}(\Omega)$ and $L^{2}(\Omega)$ respectively [17, and $C_{0}^{\infty}(\Omega) \cap V(\Omega)$ is dense in $V(\Omega)$ and $W(\Omega)$. We denote by $L_{0}^{2}(\Omega)$ the space

$$
L_{0}^{2}(\Omega)=\left\{p \in L^{2}(\Omega): \int_{\Omega} p=0\right\}
$$

equipped with the $\|\cdot\|_{L^{2}(\Omega)}$ norm. The dual spaces of $H_{0}^{1}(\Omega)$ and $V(\Omega)$ are denoted by $H^{-1}(\Omega)$ and $V(\Omega)^{*}$ respectively. The later space is defined by

$$
\|f\|_{V(\Omega)^{*}}=\sup _{v \in V(\Omega):\|v\|_{H^{1}(\Omega)} \leq 1}\langle f, v\rangle,
$$

where $\langle.,$.$\rangle denotes the duality pairing between H_{0}^{1}(\Omega)$ and $H^{-1}(\Omega)$.

For any Banach space $X$ let $L^{p}[0, T ; X], 1 \leq p<\infty$, and $L^{\infty}[0, T ; X]$ denote the spaces of $p$-integrable functions taking values in $X$ with norm

$$
\|f\|_{L^{p}[0, T ; X]}=\left(\int_{0}^{T}\|f(t)\|_{X}^{p}\right)^{1 / p}, \quad\|f\|_{L^{\infty}[0, T ; X]}=\operatorname{esssup}_{t \in[0, T]}\|f\|_{X}<\infty,
$$

and let $H^{1}[0, T ; X]$ denote the space of functions with square integrable derivatives with norm

$$
\|f\|_{H^{1}[0, T ; X]}=\left(\int_{0}^{T}\|f\|_{X}^{2} d t+\int_{0}^{T}\left\|f_{t}\right\|_{X}^{2} d t\right)^{1 / 2} .
$$

\section{WEAK STATEMENTS AND NUMERICAL SCHEME}

The numerical schemes will approximate the natural weak formulation of equation (1.1) which seeks

$$
(\mathbf{u}, p) \in L^{2}\left[0, T ; H^{1}(\Omega)\right] \cap H^{1}\left[0, T ; H^{-1}(\Omega)\right] \times L^{2}\left[0, T ; L_{0}^{2}(\Omega)\right]
$$

such that for a.e. $t \in(0, T]$,

$$
\left\{\begin{aligned}
\left\langle\mathbf{u}_{t}, \mathbf{v}\right\rangle+a(\mathbf{u}, \mathbf{v})+b(\mathbf{v}, p)+c(\mathbf{u}, \mathbf{u}, \mathbf{v}) & =\langle\mathbf{f}, \mathbf{v}\rangle & & \forall \mathbf{v} \in H_{0}^{1}(\Omega), \\
b(\mathbf{u}, q) & =0 & & \forall q \in L_{0}^{2}(\Omega), \\
(\mathbf{u}(0, x), \mathbf{v}) & =\left(\mathbf{u}_{0}, \mathbf{v}\right) & & \forall \mathbf{v} \in W(\Omega) .
\end{aligned}\right.
$$


The bilinear forms $a: H^{1}(\Omega) \times H^{1}(\Omega) \rightarrow \mathbb{R}$ and $b: H^{1}(\Omega) \times L^{2}(\Omega) \rightarrow \mathbb{R}$ are defined by

$$
a(\mathbf{u}, \mathbf{v})=\nu \int_{\Omega}(\nabla \mathbf{u}):(\nabla \mathbf{v}) \quad \text { and } \quad b(\mathbf{v}, p)=\int_{\Omega} q(\nabla \cdot \mathbf{v}),
$$

where $(\nabla \mathbf{u}):(\nabla \mathbf{v})=\sum_{i, j=1}^{d} u_{i, j} v_{i, j}$ and the index after the comma indicates the derivative with respect to $x_{j}$. The nonlinear term $c(., .,$.$) is trilinear on H^{1}(\Omega)^{d}$ and takes the form

$c(\mathbf{u}, \mathbf{v}, \mathbf{w})=(1 / 2)(\hat{c}(\mathbf{u}, \mathbf{v}, \mathbf{w})-\hat{c}(\mathbf{u}, \mathbf{w}, \mathbf{v}))$, where $\hat{c}(\mathbf{u}, \mathbf{v}, \mathbf{w})=\sum_{i, j=1}^{d} \int_{\Omega} \mathbf{u}_{j}\left(\frac{\partial \mathbf{v}_{i}}{\partial x_{j}}\right) \mathbf{w}_{i}$.

The bilinear form $a(.,$.$) is continuous and satisfies the classical coercivity condi-$ tion. In particular, there exist constants $C, c>0$, depending only on the domain $\Omega$ through the Poincaré inequality such that

$$
|a(\mathbf{u}, \mathbf{v})| \leq \nu C\|\mathbf{u}\|_{H^{1}(\Omega)}\|\mathbf{v}\|_{H^{1}(\Omega)} \quad \text { and } \quad a(\mathbf{u}, \mathbf{u}) \geq c \nu\|\mathbf{u}\|_{H^{1}(\Omega)}^{2} .
$$

The bilinear form $b(.,$.$) is also continuous and satisfies the classical inf-sup condition$ [5].

Lemma 2.1. There exist constants $C, c$ depending only on $\Omega$ such that the bilinear form $b(.,$.$) satisfies the following properties:$

(1) Continuity:

$$
b(\mathbf{v}, q) \leq C\|\mathbf{u}\|_{H^{1}(\Omega)}\|q\|_{L^{2}(\Omega)} .
$$

(2) Inf-sup condition:

$$
\inf _{q \in L_{0}^{2}(\Omega)} \sup _{\mathbf{v} \in H_{0}^{1}(\Omega)} \frac{b(\mathbf{v}, q)}{\|\mathbf{v}\|_{H_{0}^{1}(\Omega)}\|q\|_{L_{0}^{2}(\Omega)}} \geq c>0 .
$$

The trilinear forms satisfy the following continuity and skew symmetry properties 31 .

Lemma 2.2. The trilinear forms satisfy

$$
\begin{gathered}
c(\mathbf{u}, \mathbf{w}, \mathbf{v})=\hat{c}(\mathbf{u}, \mathbf{v}, \mathbf{w})=-\hat{c}(\mathbf{u}, \mathbf{w}, \mathbf{v}), \quad \mathbf{u} \in V(\Omega), \quad \mathbf{v}, \mathbf{w} \in H_{0}^{1}(\Omega), \\
c(\mathbf{u}, \mathbf{v}, \mathbf{w})=-c(\mathbf{u}, \mathbf{w}, \mathbf{v}), \quad \mathbf{u}, \mathbf{v}, \mathbf{w} \in H^{1}(\Omega),
\end{gathered}
$$

and the bounds

$$
\begin{aligned}
& |\hat{c}(\mathbf{u}, \mathbf{v}, \mathbf{w})| \leq\|\mathbf{u}\|_{L^{p}(\Omega)}\|\nabla \mathbf{v}\|_{L^{2}(\Omega)}\|\mathbf{w}\|_{L^{p^{\prime}}(\Omega)}, \quad 1 / p+1 / p^{\prime}=1 / 2 \\
& |c(\mathbf{u}, \mathbf{v}, \mathbf{w})| \leq C\|\mathbf{u}\|_{H^{1}(\Omega)}\|\mathbf{v}\|_{H^{1}(\Omega)}\|\mathbf{w}\|_{H^{1}(\Omega)}
\end{aligned}
$$

where $C>0$ depends upon the domain $\Omega$.

In two dimensions $(d=2)$

$$
\begin{aligned}
|c(\mathbf{u}, \mathbf{v}, \mathbf{w})| \leq & C\|\mathbf{u}\|_{L^{2}(\Omega)}^{1 / 2}\|\nabla \mathbf{u}\|_{L^{2}(\Omega)}^{1 / 2}\left(\|\mathbf{v}\|_{H^{1}(\Omega)}\|\mathbf{w}\|_{L^{2}(\Omega)}^{1 / 2}\|\nabla \mathbf{w}\|_{L^{2}(\Omega)}^{1 / 2}\right. \\
& \left.+\|\mathbf{w}\|_{H^{1}(\Omega)}\|\mathbf{v}\|_{L^{2}(\Omega)}^{1 / 2}\|\nabla \mathbf{v}\|_{L^{2}(\Omega)}^{1 / 2}\right)
\end{aligned}
$$

while in three dimensions $(d=3)$ [20],

$$
|c(\mathbf{u}, \mathbf{v}, \mathbf{w})| \leq C\|\mathbf{u}\|_{L^{2}(\Omega)}^{1 / 2}\|\mathbf{u}\|_{H^{1}(\Omega)}^{1 / 2}\|\mathbf{v}\|_{H^{1}(\Omega)}\|\mathbf{w}\|_{H^{1}(\Omega)} .
$$


2.1. Fully discrete formulation. To approximate the solution of (2.1) we introduce a partition $0=t^{0}<t^{1}<\cdots<t^{N}=T$ and for each interval $\left(t^{n-1}, t^{n}\right)$ discrete spaces $U_{h}^{n} \subset H_{0}^{1}(\Omega)$ for the velocity and $Q_{h}^{n} \subset L_{0}^{2}(\Omega)$ for the pressure. We let $\mathcal{P}_{k}\left[t^{n-1}, t^{n} ; U_{h}^{n}\right]$ and $\mathcal{P}_{k}\left[t^{n-1}, t^{n} ; Q_{h}^{n}\right]$ denote the set of polynomials of degree $k$ in time with values in $U_{h}^{n}$ and $Q_{h}^{n}$ respectively. On each partition $\left(t^{n-1}, t^{n}\right)$ the Discontinuous Galerkin method constructs an approximate velocity

$$
\mathbf{u}_{h} \in \mathcal{U}^{h} \equiv\left\{\mathbf{u}_{h} \in L^{2}\left[0, T ; H_{0}^{1}(\Omega)\right]:\left.\mathbf{u}_{h}\right|_{\left(t^{n-1}, t^{n}\right]} \in \mathcal{P}_{k}\left[t^{n-1}, t^{n} ; U_{h}^{n}\right]\right\}
$$

and pressure

$$
p_{h} \in \mathcal{P}^{h} \equiv\left\{p_{h} \in L^{2}\left[0, T ; L_{0}^{2}(\Omega)\right]:\left.p_{h}\right|_{\left(t^{n-1}, t^{n}\right]} \in \mathcal{P}_{k}\left[t^{n-1}, t^{n} ; Q_{h}^{n}\right]\right\}
$$

satisfying

$$
\left\{\begin{array}{c}
\left(\mathbf{u}_{-}^{n}, \mathbf{v}_{-}^{n}\right)+\int_{t^{n-1}}^{t^{n}}\left(-\left\langle\mathbf{u}, \mathbf{v}_{h t}\right\rangle+a\left(\mathbf{u}_{h}, \mathbf{v}_{h}\right)+c\left(\mathbf{u}_{h}, \mathbf{u}_{h}, \mathbf{v}_{h}\right)+b\left(\mathbf{v}_{h}, p_{h}\right)\right) \\
=\left(\mathbf{u}_{-}^{n-1}, \mathbf{v}_{+}^{n-1}\right)+\int_{t^{n-1}}^{t^{n}}\left\langle\mathbf{f}, \mathbf{v}_{h}\right\rangle \quad \forall \mathbf{v}_{h} \in \mathcal{P}_{k}\left[t^{n-1}, t^{n} ; U_{h}^{n}\right] \\
\int_{t^{n-1}}^{t^{n}} b\left(\mathbf{u}_{h}, q_{h}\right)=0 \quad \forall q_{h} \in \mathcal{P}_{k}\left[t^{n-1}, t^{n} ; Q_{h}^{n}\right] \\
\mathbf{u}_{h}(0)=\mathbf{u}_{-}^{0}
\end{array}\right.
$$

Here we write $\mathbf{u}_{ \pm}^{n}=\mathbf{u}_{h}\left(t_{ \pm}^{n}\right)$ and for the traces from above and below respectively. We assume that $U_{h}^{n}$ and $Q_{h}^{n}$ satisfy the usual approximation properties and inf-sup condition [10, 17.

Assumption 1. Let $\left\{\mathcal{T}_{h}^{n} \mid 0 \leq n \leq N_{h}, h>0\right\}$ be a regular family of triangulations of $H_{0}^{1}(\Omega)$ and let $U_{h}^{n} \subset H_{0}^{1}(\Omega)$ and $Q_{h}^{n} \subset L_{0}^{2}(\Omega)$ be finite element subspaces constructed over $\mathcal{T}_{h}^{n}$ using piecewise polynomials satisfying the following approximation properties.

There exists an integer $\ell \geq 1$ and constants $C, c>0$ independent of $h, \mathbf{u}$, and $p$ such that:

(1) If $\mathbf{u} \in H^{l+1}(\Omega) \cap H_{0}^{1}(\Omega)$, then

$$
\inf _{\mathbf{u}_{h} \in U_{h}^{n}}\left\|\mathbf{u}-\mathbf{u}_{h}\right\|_{H^{s}(\Omega)} \leq C h^{l+1-s}\|\mathbf{u}\|_{H^{l+1}(\Omega)}, \quad 0 \leq l \leq \ell, \quad s=-1,0,1 .
$$

(2) If $p \in H^{l}(\Omega) \cap L_{0}^{2}(\Omega)$, then

$$
\inf _{q_{h} \in Q_{h}^{n}}\left\|p-q_{h}\right\|_{L^{2}(\Omega)} \leq C h^{l}\|p\|_{H^{l}(\Omega)}, \quad 0 \leq l \leq \ell .
$$

(3) The subspaces $U_{h}^{n}, Q_{h}^{n}$ satisfy the classical inf-sup condition (2.5),

$$
\inf _{q_{h} \in Q_{h}^{n} \operatorname{vup}_{h} \in U_{h}^{n}} \frac{b\left(\mathbf{v}_{h}, q_{h}\right)}{\left\|\mathbf{v}_{h}\right\|_{H^{1}(\Omega)}\left\|q_{h}\right\|_{L^{2}(\Omega)}} \geq c .
$$

(4) If the triangulations are quasi-uniform, then there exists $C_{i n v}>0$ independent of $h$ such that

$$
\left\|\mathbf{u}_{h}\right\|_{H^{1}(\Omega)} \leq\left(C_{\text {inv }} / h\right)\left\|\mathbf{u}_{h}\right\|_{L^{2}(\Omega)}, \quad \forall \mathbf{u}_{h} \in U_{h}^{n} .
$$

We next define the discrete divergence free subspace of $\mathcal{P}_{k}\left[t^{n-1}, t^{n} ; U_{h}^{n}\right]$ to be

$$
\mathcal{Z}_{h}^{n} \equiv\left\{\mathbf{u}_{h} \in \mathcal{P}_{k}\left[t^{n-1}, t^{n} ; U_{h}^{n}\right] \mid \int_{t^{n-1}}^{t^{n}} b\left(\mathbf{u}_{h}, q_{h}\right)=0 \quad \forall q_{h} \in \mathcal{P}_{k}\left[t^{n-1}, t^{n} ; Q_{h}^{n}\right]\right\}
$$


The following lemma characterizes the spaces $\mathcal{Z}_{h}^{n}$ and illustrates why it is natural to choose the time variation (i.e. degree $k$ ) for the approximate pressure and velocity to be the same.

Lemma 2.3. Let $Z_{h}^{n}=\left\{\mathbf{u}_{h} \in U_{h}^{n}: b\left(\mathbf{u}_{h}, q_{h}\right)=0, \forall q_{h} \in Q_{h}^{n}\right\}$, then $\mathcal{Z}_{h}^{n}=$ $\mathcal{P}_{k}\left[t^{n-1}, t^{n} ; Z_{h}^{n}\right]$.

Proof. It is clear that $\mathcal{P}_{k}\left[t^{n-1}, t^{n} ; Z_{h}^{n}\right] \subset \mathcal{Z}_{h}^{n}$. It suffices to show the reverse inclusion. Let $\left\{\eta_{i}\right\}_{i=0}^{k} \subset \mathcal{P}_{k}\left[t^{n-1}, t^{n}\right]$ be an orthonormal basis with the $L^{2}\left[t^{n-1}, t^{n}\right]$ inner product. Then write $\mathbf{u}_{h} \in \mathcal{P}_{k}\left[t^{n-1}, t^{n} ; U_{h}^{n}\right]$ and $q_{h} \in \mathcal{P}_{k}\left[t^{n-1}, t^{n} ; Q_{h}^{n}\right]$ as

$$
\mathbf{u}_{h}=\sum_{i=0}^{k} \eta_{i} \mathbf{u}_{i} \quad \text { and } \quad q_{h}=\sum_{j=0}^{k} \eta_{j} q_{j}
$$

Since $b(.,):. U_{h}^{n} \times Q_{h}^{n} \rightarrow \mathbb{R}$ is bilinear (and independent of time),

$$
\int_{t^{n-1}}^{t^{n}} b\left(\mathbf{u}_{h}, q_{h}\right)=\sum_{i, j=0}^{k} \int_{t^{n-1}}^{t^{n}} \eta_{i} \eta_{j} b\left(\mathbf{u}_{i}, q_{j}\right)=\sum_{i=0}^{k} b\left(\mathbf{u}_{i}, q_{i}\right) .
$$

If $\mathbf{u}_{h} \in \mathcal{Z}_{h}^{n}$, the left-hand expression vanishes for all $\left\{q_{j}\right\}_{j=0}^{k} \subset Q_{h}^{n}$. Therefore, $\mathbf{u}_{i} \in Z_{h}^{n}$ for each $0 \leq i \leq k$, and since $Z_{h}^{n}$ is a linear space it follows that $\mathbf{u}_{h} \in$ $\mathcal{P}_{k}\left[t^{n-1}, t^{n} ; Z_{h}^{n}\right]$.

This lemma allows us to write

$$
\begin{aligned}
\mathcal{Z}^{h} & \equiv\left\{\mathbf{u}_{h} \in \mathcal{U}^{h} \mid \int_{0}^{T} b\left(\mathbf{u}_{h}, q_{h}\right)=0, \forall q_{h} \in \mathcal{P}^{h}\right\} \\
& =\left\{\mathbf{u}_{h} \in \mathcal{U}^{h}\left|\mathbf{u}_{h}\right|_{\left(t^{n-1}, t^{n}\right]} \in \mathcal{Z}_{h}^{n}\right\} \\
& =\left\{\mathbf{u}_{h} \in \mathcal{U}^{h}\left|\mathbf{u}_{h}\right|_{\left(t^{n-1}, t^{n}\right]} \in \mathcal{P}_{k}\left[t^{n-1}, t^{n} ; Z_{h}^{n}\right]\right\}
\end{aligned}
$$

\section{Discrete Characteristic FunCtions And Interpolants}

In this section we present some elementary properties of polynomials required to establish stability and error estimates for the DG approximations of the Stokes and Navier-Stokes equations.

3.1. Discrete characteristic functions. Estimates for evolution equations are frequently obtained by multiplying the equation by $\mathbf{u}$ and integrating to an arbitrary time $t \in[0, T]$. This amounts to selecting the test function to be $\chi_{[0, t)} \mathbf{u}$, and this choice is not available in the discrete context unless the terminal time is one of the partition points. In the past this problem has been circumvented by deriving bounds for certain temporal derivatives of the solution, so that the solution between the partition points was effectively controlled by values at these points [33. This approach is problematic for the (Navier-) Stokes equations since the time derivative of the pressure is usually not available, and it is difficult to get separate estimates for $\mathbf{u}_{t}$ and $\nabla p$ given a bound on $\mathbf{u}_{t}-\nabla p$ in $H^{-1}(\Omega)$.

To estimate the solution at times $t \in\left[t^{n-1}, t^{n}\right)$ we first recall the discrete characteristic functions as introduced in [7, Section 2.3], and then extend the construction in [7, 8] to accommodate (discretely) divergence free subspaces. The discrete characteristic functions on each interval are invariant under translation so it is convenient to work on the interval $[0, \tau)$ with $\tau=t^{n}-t^{n-1}$. We begin by considering 
polynomials $r \in \mathcal{P}_{k}[0, \tau]$. A discrete approximation of $\chi_{[0, t)} r$ is the polynomial $\tilde{r} \in\left\{\tilde{r} \in \mathcal{P}_{k}[0, \tau] \mid \tilde{r}(0)=r(0)\right\}$ satisfying

$$
\int_{0}^{\tau} \tilde{r} q=\int_{0}^{t} r q \quad \forall q \in \mathcal{P}_{k-1}[0, \tau] .
$$

The above construction is motivated by the fact that we may put $q=r^{\prime}$ to obtain $\int_{0}^{\tau} \tilde{r} r^{\prime}=\int_{0}^{t} r r^{\prime}=(1 / 2)\left(r^{2}(t)-r^{2}(0)\right)$.

We next extend this elementary construction to approximate functions of the form $\chi_{[0, t)} \mathbf{v}$ for $\mathbf{v} \in \mathcal{P}_{k}[0, \tau ; V]$ where $V$ is any semi-inner product space. If $\mathbf{v} \in$ $\mathcal{P}_{k}[0, \tau ; V]$, we can write $\mathbf{v}=\sum_{i=0}^{k} r_{i}(t) \mathbf{v}_{i}$ where $\left\{r_{i}\right\} \subset \mathcal{P}_{k}[0, \tau]$ and $\left\{\mathbf{v}_{i}\right\} \subset V$. If we define $\tilde{\mathbf{v}}=\sum_{i=0}^{k} \tilde{r}_{i}(t) \mathbf{v}_{i}$, it is clear that $\tilde{\mathbf{v}} \in \mathcal{P}_{k}[0, \tau ; V]$ satisfies

$$
\tilde{\mathbf{v}}(0)=\mathbf{v}(0) \text { and } \int_{0}^{\tau}(\tilde{\mathbf{v}}, \mathbf{w})_{V}=\int_{0}^{t}(\mathbf{v}, \mathbf{w})_{V} \quad \forall \mathbf{w} \in \mathcal{P}_{k-1}[0, \tau ; V] .
$$

The following elementary lemma from [7, Lemma 2.7] shows that the mapping $\mathbf{v} \mapsto \tilde{\mathbf{v}}$ is continuous on $\mathcal{P}_{k}[0, \tau, V]$.

Lemma 3.1. Let $V$ be a semi-inner product space, then the mapping

$$
\mathbf{v}=\sum_{i=0}^{k} r_{i}(t) \mathbf{v}_{i} \mapsto \tilde{\mathbf{v}}=\sum_{i=0}^{k} \tilde{r}_{i}(t) \mathbf{v}_{i}
$$

on $\mathcal{P}_{k}[0, \tau ; V]$ is continuous in $\|\cdot\|_{L^{2}[0, \tau ; V]}$. In particular, there exists $C_{k}>0$ depending only upon $k$ such that

$$
\|\tilde{\mathbf{v}}\|_{L^{2}[0, \tau ; V]} \leq C_{k}\|\mathbf{v}\|_{L^{2}[0, \tau ; V]} \text { and }\left\|\tilde{\mathbf{v}}-\chi_{[0, t)} \mathbf{v}\right\|_{L^{2}[0, \tau ; V]} \leq C_{k}\|\mathbf{v}\|_{L^{2}[0, \tau ; V]} .
$$

Notice that the above construction is purely algebraic in the sense that equation (3.1) holds for any choice of inner product on $V$. The following lemma gives an explicit formula, $\tilde{\mathbf{v}}(s)=\phi(s) \mathbf{z}$, when $\mathbf{v}(s)=\mathbf{z}$ is constant.

Lemma 3.2. Fix $t \in[0, \tau]$ and let $p \in \mathcal{P}_{k}[0, \tau]$ be characterized by

$$
p(0)=1, \quad \int_{0}^{\tau} p q=\int_{0}^{t} q, \quad q \in \mathcal{P}_{k-1}[0, \tau] .
$$

Then

$$
p(s)=1+(s / \tau) \sum_{i=0}^{k-1} c_{i} \hat{p}_{i}(s / \tau), \quad c_{i}=\int_{t / \tau}^{1} \hat{p}_{i}(\eta) d \eta,
$$

where $\left\{\hat{p}_{i}\right\}_{i=0}^{k-1}$ is an orthonormal basis of $\mathcal{P}_{k-1}[0,1]$ in the (weighted) space $L_{w}^{2}[0,1]$ having inner product

$$
(\hat{p}, \hat{q})=\int_{0}^{1} \eta \hat{p}(\eta) \hat{q}(\eta) d \eta
$$

In particular,

$$
\|p\|_{L^{\infty}[0, \tau]} \leq 1+(1-t / \tau) \sum_{i=0}^{k-1}\left\|\hat{p}_{i}\right\|_{L^{\infty}[0,1]}^{2} \leq C_{k},
$$

with $C_{k}$ independent of $t \in[0, \tau]$. 
3.2. An exponential interpolant. Bounds for DG approximations of the Stokes equations in $L^{\infty}\left[0, T ; H^{1}(\Omega)\right]$ will be obtained using the polynomial interpolant of $e^{-\lambda\left(t-t^{n-1}\right)} \mathbf{u}_{h}$ introduced in this section.

Definition 3.3. Let $V$ be a linear space, and $\lambda>0$ be given. If $\mathbf{v}=\sum_{i=0}^{k} r_{i}(t) \mathbf{v}_{i} \in$ $\mathcal{P}_{k}\left[t^{n-1}, t^{n} ; V\right]$, where $r_{i} \in \mathcal{P}_{k}\left[t^{n-1}, t^{n}\right], \mathbf{v}_{i} \in V$, we define the exponential interpolant of $\mathbf{v}$ by

$$
\overline{\mathbf{v}}=\sum_{i=0}^{k} \bar{r}_{i}(t) \mathbf{v}_{i}
$$

where $\bar{r}_{i} \in \mathcal{P}_{k}\left[t^{n-1}, t^{n}\right]$ is the approximation of $r_{i}(t) e^{-\lambda\left(t-t^{n-1}\right)}$ satisfying $\bar{r}_{i}\left(t^{n-1}\right)=$ $r_{i}\left(t^{n-1}\right)$ and

$$
\int_{t^{n-1}}^{t^{n}} \bar{r}_{i}(t) q(t) d t=\int_{t^{n-1}}^{t^{n}} r_{i}(t) q(t) e^{-\lambda\left(t-t^{n-1}\right)} d t, \quad q \in \mathcal{P}_{k-1}\left[t^{n-1}, t^{n}\right] .
$$

Note that the map $\mathbf{v} \rightarrow \overline{\mathbf{v}}$ does not define a projection, since $\overline{\overline{\mathbf{v}}} \neq \overline{\mathbf{v}}$, so we will refer to it as an exponential interpolant. Unlike the polynomial approximations of $\chi_{\left[t^{n-1}, t\right)} \mathbf{u}_{h}$ introduced above, the difference between the interpolant $\overline{\mathbf{v}}$ and $\mathbf{v}$ remains small.

Lemma 3.4. Let $V$ and $Q$ be linear spaces and $\mathbf{v} \mapsto \overline{\mathbf{v}}$ the map constructed in Definition 3.3 with parameter $\lambda>0$. If $L(.,):. V \times Q \rightarrow \mathbb{R}$ is a bilinear mapping and $\mathbf{v} \in \mathcal{P}_{k}\left[t^{n-1}, t^{n} ; V\right]$, then

$$
\int_{t^{n-1}}^{t^{n}} L(\overline{\mathbf{v}}(t), q(t)) d t=\int_{t^{n-1}}^{t^{n}} L(\mathbf{v}(t), q(t)) e^{-\lambda\left(t-t^{n-1}\right)} d t, \quad q \in \mathcal{P}_{k-1}\left[t^{n-1}, t^{n} ; Q\right] .
$$

If $(.,)_{V}$ is a (semi-)inner product on $V$, then there exists a constant $C_{k}>0$ independent of $\lambda \geq 0$ such that

$$
\begin{aligned}
\|\mathbf{v}-\overline{\mathbf{v}}\|_{L^{2}\left[t^{n-1}, t^{n} ; V\right]} & \leq C_{k}\left(1-e^{-\lambda\left(t^{n}-t^{n-1}\right)}\right)\|\mathbf{v}\|_{L^{2}\left[t^{n-1}, t^{n} ; V\right]} \\
& \leq C_{k} \lambda\left(t^{n}-t^{n-1}\right)\|\mathbf{v}\|_{L^{2}\left[t^{n-1}, t^{n} ; V\right]}
\end{aligned}
$$

in particular,

$$
\|\overline{\mathbf{v}}\|_{L^{2}\left[t^{n-1}, t^{n} ; V\right]} \leq\left(1+C_{k}\right)\|\mathbf{v}\|_{L^{2}\left[t^{n-1}, t^{n} ; V\right]} .
$$

Proof (Sketch). The proof follows along the lines of [7, Lemma 2.3,2.4]. The key step is to prove that

$$
\int_{t^{n-1}}^{t^{n}}\left(r_{i}-\bar{r}_{i}\right)^{2} \leq C_{k}\left(1-e^{-\lambda\left(t^{n}-t^{n-1}\right)}\right) \int_{t^{n-1}}^{t^{n}} r_{i}^{2}
$$

For that purpose, note that since $r_{i}\left(t^{n-1}\right)=\bar{r}_{i}\left(t^{n-1}\right)$ we may write $r_{i}-\bar{r}_{i}=$ $\left(t-t^{n-1}\right) p_{i}$, for some $p_{i} \in \mathcal{P}_{k-1}\left[t^{n-1}, t^{n}\right]$. Using the definition of the interpolant we derive

$$
\int_{t^{n-1}}^{t^{n}}\left(t-t^{n-1}\right) p_{i} q=\int_{t^{n-1}}^{t^{n}}\left(1-e^{-\lambda\left(t-t^{n-1}\right)}\right) r_{i} q \quad \forall q \in \mathcal{P}_{k-1}\left[t^{n-1}, t^{n}\right] .
$$

Selecting $q=p_{i}$ and using the equivalence of norms on the $\mathcal{P}_{k}\left[t^{n-1}, t^{n}\right]$ shows

$$
\|p\|_{L^{2}\left[t^{n-1}, t^{n}\right]} \leq C(k)\left(1-e^{-\lambda\left(t^{n}-t^{n-1}\right)}\right)\left\|r_{i}\right\|_{L^{2}\left[t^{n-1}, t^{n}\right]} .
$$

The remaining calculations are elementary; see [7, Lemma 2.4]. 
A classical scaling argument and the finite dimensionality of $\mathcal{P}_{k}\left[t^{n-1}, t^{n}\right]$ implies the following lemma.

Lemma 3.5. Let $V$ be a linear space and (.,. $)_{V}$ a (semi-)inner product on $V$ and let $w(t) \geq 0$ be a nonzero element of $L^{1}\left[t^{n-1}, t^{n}\right]$ and set $\tau=t^{n}-t^{n-1}$. Then, there exists a constant $C_{k}>0$ depending only on $k$ and $w$ such that

$$
\|\mathbf{v}\|_{L^{p}\left[t^{n-1}, t^{n} ; V\right]} \leq \tau^{1 / p-1 / 2}\left(C_{k} \int_{t^{n-1}}^{t^{n}} w\left(\left(t-t^{n-1}\right) / \tau\right)\|\mathbf{v}(t)\|_{V}^{2} d t\right)^{1 / 2}
$$

for all $\mathbf{v} \in \mathcal{P}_{k}\left[t^{n-1}, t^{n} ; V\right]$ and $1 \leq p \leq \infty$. In particular, if $(1 / p)+\left(1 / p^{\prime}\right)=1$,

$$
\|\mathbf{v}\|_{L^{p}\left[t^{n-1}, t^{n} ; V\right]}\|\mathbf{v}\|_{L^{p^{\prime}\left[t^{n-1}, t^{n} ; V\right]}} \leq C_{k} \int_{t^{n-1}}^{t^{n}} w\left(\left(t-t^{n-1}\right) / \tau\right)\|\mathbf{v}(t)\|_{V}^{2} d t .
$$

This inverse estimate can be used to bound $\mathbf{v}-\overline{\mathbf{v}}$ in $L^{p}\left[t^{n-1}, t^{n} ; V\right]$ for any $p \geq 1$.

Lemma 3.6. Let $V$ be a linear space and $(., .)_{V}$ a semi-inner product on $V$ and let $\mathbf{v} \mapsto \overline{\mathbf{v}}$ denote the exponential interpolant on $\mathcal{P}_{k}\left[t^{n-1}, t^{n} ; V\right]$ constructed in Definition 3.3. Set $\tau=t^{n}-t^{n-1}$, then there exists a constant $C_{k}>0$ depending only on $k$ such that

$$
\|\mathbf{v}-\overline{\mathbf{v}}\|_{L^{p}\left[t^{n-1}, t^{n} ; V\right]} \leq C_{k}\left(1-e^{\lambda \tau}\right)\|\mathbf{v}\|_{L^{p}\left[t^{n-1}, t^{n} ; V\right]} \leq C_{k} \lambda \tau\|\mathbf{v}\|_{L^{p}\left[t^{n-1}, t^{n} ; V\right]},
$$

for all $\mathbf{v} \in \mathcal{P}_{k}\left[t^{n-1}, t^{n} ; V\right]$ and $1 \leq p \leq \infty$. In particular,

$$
\|\overline{\mathbf{v}}\|_{L^{p}\left[t^{n-1}, t^{n} ; V\right]} \leq\left(1+C_{k}\right)\|\mathbf{v}\|_{L^{p}\left[t^{n-1}, t^{n} ; V\right]} .
$$

Proof. Using Lemma 3.5 with $w(t) \equiv 1$ and Lemma 3.4 we obtain,

$$
\begin{aligned}
\|\mathbf{v}-\overline{\mathbf{v}}\|_{L^{p}\left[t^{n-1}, t^{n} ; V\right]} & \leq \tau^{1 / p-1 / 2} C_{k}\|\mathbf{v}-\overline{\mathbf{v}}\|_{L^{2}\left[t^{n-1}, t^{n} ; V\right]} \\
& \leq \tau^{1 / p-1 / 2} C_{k}\left(1-e^{\lambda \tau}\right)\|\mathbf{v}\|_{L^{2}\left[t^{n-1}, t^{n} ; V\right]}
\end{aligned}
$$

If $1 / p+1 / p^{\prime}=1$, Hölder's inequality and another application of Lemma 3.5 shows

$$
\begin{aligned}
\|\mathbf{v}\|_{L^{2}\left[t^{n-1}, t^{n} ; V\right]}^{2} & \leq\|\mathbf{v}\|_{L^{p}\left[t^{n-1}, t^{n} ; V\right]}\|\mathbf{v}\|_{L^{p^{\prime}\left[t^{n-1}, t^{n} ; V\right]}} \\
& \leq\|\mathbf{v}\|_{L^{p}\left[t^{n-1}, t^{n} ; V\right]} C_{k} \tau^{1 / p^{\prime}-1 / 2}\|\mathbf{v}\|_{L^{2}\left[t^{n-1}, t^{n} ; V\right]}
\end{aligned}
$$

So

$$
\|\mathbf{v}\|_{L^{2}\left[t^{n-1}, t^{n} ; V\right]} \leq C_{k} \tau^{1 / p^{\prime}-1 / 2}\|\mathbf{v}\|_{L^{p}\left[t^{n-1}, t^{n} ; V\right]}=C_{k} \tau^{1 / 2-1 / p}\|\mathbf{v}\|_{L^{p}\left[t^{n-1}, t^{n} ; V\right]}
$$

and the lemma follows.

\section{DG SCHEME FOR AN AUXILIARY PDE}

In this section we introduce a "parabolic" projection $\left(\mathbb{P}_{h}\right.$ below) that will be used to construct test functions for the DG scheme used to approximate the NavierStokes equations. It is designed to circumvent the difficulties associated with the use of different spaces for each time step and the jump terms that appear in the DG scheme. We consider DG approximations for the evolutionary Stokes equations; that is,

$$
(\mathbf{u}, r) \in L^{2}\left[0, T ; H_{0}^{1}(\Omega)\right] \cap H^{1}\left[0, T ; H^{-1}(\Omega)\right] \times L^{2}\left[0, T ; L_{0}^{2}(\Omega)\right]
$$

and

$$
\left\{\begin{aligned}
\left\langle\mathbf{u}_{t}, \mathbf{v}\right\rangle+a(\mathbf{u}, \mathbf{v})+b(\mathbf{v}, r) & =\langle\mathbf{f}, \mathbf{v}\rangle & & \forall \mathbf{v} \in H_{0}^{1}(\Omega), \\
b(\mathbf{u}, q) & =0 & & \forall q \in L_{0}^{2}(\Omega), \\
(\mathbf{u}(0), \mathbf{w}) & =\left(\mathbf{u}_{0}, \mathbf{w}\right) & & \forall \mathbf{w} \in W(\Omega) .
\end{aligned}\right.
$$


The numerical schemes are based upon the weak formulation: $\mathbf{u} \in L^{2}\left[0, T ; H_{0}^{1}(\Omega)\right] \cap$ $H^{1}\left[0, T ; H^{-1}(\Omega)\right] \hookrightarrow C\left[0, T ; L^{2}(\Omega)\right], r \in L^{2}\left[0, T ; L_{0}^{2}(\Omega)\right]$ and

$$
\left\{\begin{array}{c}
(\mathbf{u}(T), \mathbf{v}(T))+\int_{0}^{T}-\left\langle\mathbf{u}, \mathbf{v}_{t}\right\rangle+a(\mathbf{u}, \mathbf{v})+b(\mathbf{v}, r) \\
=(\mathbf{u}(0), \mathbf{v}(0))+\int_{0}^{T}\langle\mathbf{f}, \mathbf{v}\rangle \quad \forall \mathbf{v} \in L^{2}\left[0, T ; H_{0}^{1}(\Omega)\right] \\
\cap H^{1}\left[0, T ; H^{-1}(\Omega)\right] \\
\int_{0}^{T} b(\mathbf{u}, q)=0 \quad \forall q \in L^{2}\left[0, T ; L_{0}^{2}(\Omega)\right]
\end{array}\right.
$$

Given a partition $0=t^{0}<t^{1}<\cdots<t^{N}=T$ of $[0, T]$ and a collection $\left\{U_{h}^{n}\right\}_{n=0}^{N}$, $\left\{Q_{h}^{n}\right\}_{n=0}^{N}$ of subspaces of $H_{0}^{1}(\Omega)$ and $L_{0}^{2}(\Omega)$, respectively, the DG method constructs an approximate solution $u_{h} \in \mathcal{U}^{h}$, and a pressure $p_{h} \in \mathcal{P}^{h}$ satisfying

$$
\left\{\begin{array}{c}
\left(\mathbf{u}_{-}^{n}, \mathbf{v}_{-}^{n}\right)+\int_{t^{n-1}}^{t^{n}}\left(-\left\langle\mathbf{u}_{h}, \mathbf{v}_{h t}\right\rangle+a\left(\mathbf{u}_{h}, \mathbf{v}_{h}\right)+b\left(\mathbf{v}_{h}, r_{h}\right)\right) \\
-\left(\mathbf{u}_{-}^{n-1}, \mathbf{v}_{+}^{n-1}\right)=\int_{t^{n-1}}^{t^{n}}\left\langle\mathbf{f}, \mathbf{v}_{h}\right\rangle \quad \forall \mathbf{v}_{h} \in \mathcal{P}_{k}\left[t^{n-1}, t^{n} ; U_{h}^{n}\right] \\
\int_{t^{n-1}}^{t^{n}} b\left(\mathbf{u}_{h}, q_{h}\right)=0 \quad \forall q_{h} \in \mathcal{P}_{k}\left[t^{n-1}, t^{n} ; Q_{h}^{n}\right] .
\end{array}\right.
$$

4.1. Projections. Projections onto the discretely divergence subspaces play an important role in the analysis of numerical schemes for both the Stokes and NavierStokes equations. Here we define our "parabolic" projection, $\mathbb{P}_{h}$, and compare it with the classical Stokes projection $\Pi_{h}$.

Definition 4.1. $\quad$ (1) $P_{n}: L^{2}(\Omega) \rightarrow Z_{h}^{n}$ denotes the projection onto the discretely divergence free subspace $Z_{h}^{n}=\left\{\mathbf{u}_{h} \in U_{h}^{n} \mid b\left(\mathbf{u}_{h}, q_{h}\right)=0, q_{h} \in Q_{h}^{n}\right\}$; that is,

$$
P_{n} \mathbf{w} \in Z_{h}^{n}, \quad\left(P_{n} \mathbf{w}, \mathbf{v}_{h}\right)=\left(\mathbf{w}, \mathbf{v}_{h}\right) \quad \forall \mathbf{v}_{h} \in Z_{h}^{n} .
$$

(2) The Stokes projection $\Pi_{n}: H_{0}^{1}(\Omega) \rightarrow U_{h}^{n}$ is the first component of the pair $\left(\mathbf{u}_{h}, r_{h}\right) \in U_{h}^{n} \times Q_{h}^{n}$ satisfying

$$
a\left(\mathbf{u}_{h}, \mathbf{v}_{h}\right)+b\left(\mathbf{v}_{h}, r_{h}\right)=a\left(\mathbf{u}, \mathbf{v}_{h}\right), \quad b\left(\mathbf{u}_{h}, q_{h}\right)=0,
$$

for all $\left(\mathbf{v}_{h}, q_{h}\right) \in U_{h}^{n} \times Q_{h}^{n}$.

Formally, $\Pi_{n} \mathbf{u}$ is the Galerkin approximation of the stationary Stokes problem with the right-hand side $\mathbf{f}=-\nu \Delta \mathbf{u}$. The parabolic version introduced next is the DG approximation of the evolutionary Stokes' problem with the right-hand side $\mathbf{f}=\mathbf{u}_{t}-\nu \Delta \mathbf{u}$.

Definition 4.2. (1) The projection $\mathbb{P}_{n}^{l o c}: C\left[t^{n-1}, t^{n} ; L^{2}(\Omega)\right] \rightarrow \mathcal{P}_{k}\left[t^{n-1}, t^{n} ; Z_{h}^{n}\right]$ satisfies $\left(\mathbb{P}_{n}^{l o c} \mathbf{u}\right)^{n}=P_{n} \mathbf{u}\left(t^{n}\right)$, and

$$
\int_{t^{n-1}}^{t^{n}}\left(\mathbf{u}-\mathbb{P}_{n}^{l o c} \mathbf{u}, \mathbf{v}_{h}\right)=0, \quad \forall \mathbf{v}_{h} \in \mathcal{P}_{k-1}\left[t^{n-1}, t^{n} ; Z_{h}^{n}\right]
$$

Here we have used the convention $\left(\mathbb{P}_{n}^{\text {loc }} \mathbf{u}\right)^{n} \equiv\left(\mathbb{P}_{n}^{\text {loc }} \mathbf{u}\right)\left(t^{n}\right)$.

(2) The projection $\mathbb{P}_{h}^{l o c}: C\left[0, T ; L^{2}(\Omega)\right] \rightarrow \mathcal{Z}^{h}$ satisfies

$$
\mathbb{P}_{h}^{l o c} \mathbf{u} \in \mathcal{Z}^{h} \quad \text { and }\left.\quad\left(\mathbb{P}_{h}^{l o c} \mathbf{u}\right)\right|_{\left(t^{n-1}, t^{n}\right]}=\mathbb{P}_{n}^{l o c}\left(\left.\mathbf{u}\right|_{\left[t^{n-1}, t^{n}\right]}\right)
$$


(3) $\mathbb{P}_{h}: H^{1}\left[0, T ; H^{-1}(\Omega)\right] \cap L^{2}\left[0, T ; H_{0}^{1}(\Omega)\right] \rightarrow \mathcal{Z}^{h}$ is the velocity component of the discontinuous Galerkin solution $\left(\mathbf{u}_{h}, r_{h}\right)$ of (4.3) with $\mathbf{f}=\mathbf{u}_{t}-\nu \Delta \mathbf{u}$, and initial data $\mathbf{u}_{-}^{0}=P_{0}(\mathbf{u}(0))$.

The projections $\mathbb{P}_{n}^{l o c}$ are local in time, and the approximation error of the DG scheme for the Navier-Stokes equations will ultimately be bounded by projection errors of the form $\mathbf{u}-\mathbb{P}_{n}^{l o c} \mathbf{u}$. The following theorem, adapted from [9, bounds this error.

Theorem 4.3. Let $Z_{h}^{n} \subset H_{0}^{1}(\Omega)$ be a finite dimensional subspace and $P_{n}: L^{2}(\Omega) \rightarrow$ $Z_{h}^{n}$ be the orthogonal projection. Let $(., .)_{V}$ be a (semi-)inner product on $H_{0}^{1}(\Omega)$, then there exists a constant $C_{k}$, depending only on $k \geq 1$, such that

$$
\begin{aligned}
\left\|\mathbf{u}-\mathbb{P}_{n}^{l o c} \mathbf{u}\right\|_{L^{p}\left[t^{n-1}, t^{n} ; V\right]} \leq & \left\|\mathbf{u}-P_{n} \mathbf{u}\right\|_{L^{p}\left[t^{n-1}, t^{n} ; V\right]} \\
& +C_{k}\left(t^{n}-t^{n-1}\right)^{k+1}\left\|P_{n} \mathbf{u}^{(k+1)}\right\|_{L^{p}\left[t^{n-1}, t^{n} ; V\right]},
\end{aligned}
$$

for all $1 \leq p \leq \infty$. In particular, if the restriction of $P_{n}$ to $\left(V,\|\cdot\|_{V}\right)$ is stable, in the sense that $\left\|P_{n} \mathbf{u}\right\|_{V} \leq C\|\mathbf{u}\|_{V}$, then

$$
\begin{aligned}
\left\|\mathbf{u}-\mathbb{P}_{n}^{l o c} \mathbf{u}\right\|_{L^{p}\left[t^{n-1}, t^{n} ; V\right]} \leq & C\left\|\mathbf{u}-\mathbf{w}_{h}\right\|_{L^{p}\left[t^{n-1}, t^{n} ; V\right]} \\
& +C\left(t^{n}-t^{n-1}\right)^{k+1}\left\|\mathbf{u}^{(k+1)}\right\|_{L^{p}\left[t^{n-1}, t^{n} ; V\right]},
\end{aligned}
$$

for any $\mathbf{w}_{h} \in L^{p}\left[t^{n-1}, t^{n} ; Z_{h}^{n}\right]$. Here $\mathbf{u}^{(k+1)}$ denotes the $(k+1)$ th time derivative of u.

Approximation properties of $P_{n}$ depend crucially on the inf-sup condition for the pair $\left(U_{h}^{n}, Q_{h}^{n}\right)$. We briefly recall the development of the projection estimates for the Stokes projection since similar arguments will be developed in the parabolic setting.

Lemma 4.4. Let $a: H_{0}^{1}(\Omega) \times H_{0}^{1}(\Omega) \rightarrow \mathbb{R}$ and let $b: H_{0}^{1}(\Omega) \times L^{2}(\Omega) / \mathbb{R} \rightarrow \mathbb{R}$ be the bilinear forms introduced in Section 2, and let the subspaces $U_{h}^{n} \subset H_{0}^{1}(\Omega)$ and $Q_{h}^{n} \subset L^{2}(\Omega) / \mathbb{R}$ satisfy the inf-sup condition. Define the Stokes projection $\Pi_{n}: H_{0}^{1}(\Omega) \rightarrow U_{h}^{n}$ to be the first component of the pair $\left(\mathbf{u}_{h}, r_{h}\right) \in U_{h}^{n} \times Q_{h}^{n}$ satisfying

$$
a\left(\mathbf{u}_{h}, \mathbf{v}_{h}\right)+b\left(\mathbf{v}_{h}, r_{h}\right)=a\left(\mathbf{u}, \mathbf{v}_{h}\right), \quad b\left(\mathbf{u}_{h}, q_{h}\right)=0,
$$

for all $\left(\mathbf{v}_{h}, q_{h}\right) \in U_{h}^{n} \times Q_{h}^{n}$. If $\mathbf{u} \in V(\Omega)$ (that is $\operatorname{div}(\mathbf{u})=0$ ), then there is a constant $C>0$ independent of $\nu$ such that

$$
\left\|\mathbf{u}-\Pi_{n} \mathbf{u}\right\|_{H^{1}(\Omega)} \leq C \inf _{\mathbf{v}_{h} \in U_{h}^{n}}\left\|\mathbf{u}-\mathbf{v}_{h}\right\|_{H^{1}(\Omega)} .
$$

If, additionally, $\Omega$ is sufficiently regular to admit $H^{2}(\Omega) \times H^{1}(\Omega)$ regularity of the Stokes operator and $\left(U_{h}^{n}, Q_{h}^{n}\right)$ contain the piecewise linear functions over a regular mesh, then

$$
\left\|\mathbf{u}-\Pi_{n} \mathbf{u}\right\|_{L^{2}(\Omega)} \leq C h \inf _{\mathbf{v}_{h} \in U_{h}^{n}}\left\|\mathbf{u}-\mathbf{v}_{h}\right\|_{H^{1}(\Omega)},
$$

where $h$ is the diameter of the largest element of the mesh.

Since $\left\|\mathbf{u}-P_{n} \mathbf{u}\right\|_{L^{2}(\Omega)} \leq\left\|\mathbf{u}-\Pi_{n} \mathbf{u}\right\|_{L^{2}(\Omega)}$ it follows that the projection error $\mathbf{u}-P_{n} \mathbf{u}$ will exhibit optimal rates of convergence in $L^{2}(\Omega)$. We summarize this and other properties needed for the analysis below in the following corollary.

Corollary 4.5. Under the hypotheses of the lemma (in particular, $\mathbf{u} \in V(\Omega)$ ),

(1) $\left\|\mathbf{u}-P_{n} \mathbf{u}\right\|_{L^{2}(\Omega)} \leq C h \inf _{\mathbf{v}_{h} \in U_{h}^{n}}\left\|\mathbf{u}-\mathbf{v}_{h}\right\|_{H^{1}(\Omega)}$. 
(2) If the mesh is quasi-uniform so that the inverse estimate $\left\|\mathbf{u}_{h}\right\|_{H^{1}(\Omega)} \leq$ $(C / h)\left\|\mathbf{u}_{h}\right\|_{L^{2}(\Omega)}$ holds for $\mathbf{u}_{h} \in U_{h}^{n}$, then

$$
\left\|\mathbf{u}-P_{n} \mathbf{u}\right\|_{H^{1}(\Omega)} \leq C \inf _{\mathbf{v}_{h} \in U_{h}^{n}}\left\|\mathbf{u}-\mathbf{v}_{h}\right\|_{H^{1}(\Omega)} .
$$

(3) If $\left(U_{h}^{m}, Q_{h}^{m}\right)$ and $\left(U_{h}^{n}, Q_{h}^{n}\right)$ are two spaces satisfying the inf-sup condition over two meshes, then

$$
\left\|P_{n}\left(I-P_{m}\right) \mathbf{u}\right\|_{H^{-1}(\Omega)} \leq C h\left\|\mathbf{u}-P_{m} \mathbf{u}\right\|_{L^{2}(\Omega)} \leq C h^{2} \inf _{\mathbf{v}_{h} \in U_{h}^{m}}\left\|\mathbf{u}-\mathbf{v}_{h}\right\|_{H^{1}(\Omega)},
$$

where $h$ is the maximal diameter of an element in the two meshes.

Proofs of Lemma 4.4 and Corollary 4.5 are sketched in Appendix B

4.2. Projection error in $L^{\infty}\left[0, T ; L^{2}(\Omega)\right] \cap L^{2}\left[0, T ; H^{1}(\Omega)\right]$. The following theorem estimates the error at the partition points and is the analog of [7, Theorem $2.2]$.

Theorem 4.6. Let $\mathbf{u} \in H^{1}\left[0, T ; H^{-1}(\Omega)\right] \cap L^{2}\left[0, T ; H_{0}^{1}(\Omega)\right]$ and $\mathbf{u}_{h}=\mathbb{P}_{h} \mathbf{u}$. Then the error $\mathbf{e}_{h}=\mathbb{P}_{h}^{\text {loc }} \mathbf{u}-\mathbf{u}_{h}$ satisfies

$$
\begin{aligned}
\left\|\mathbf{e}_{h-}^{n}\right\|_{L^{2}(\Omega)}^{2}+\nu \int_{0}^{t^{n}}\left\|\mathbf{e}_{h}\right\|_{H^{1}(\Omega)}^{2}+1 / 2 \sum_{i=0}^{n-1}\left|\left[\mathbf{e}_{h}^{i}\right]\right|_{L^{2}(\Omega)}^{2} \leq C\left(\int_{0}^{t^{n}} \nu\left\|\left(I-\mathbb{P}_{h}^{l o c}\right) \mathbf{u}\right\|_{H^{1}(\Omega)}^{2}\right. \\
\left.+\sum_{i=0}^{n-1} \min \left(C_{k} /\left(\Delta t^{i+1} \nu\right)\left\|P_{i+1}\left(I-P_{i}\right) \mathbf{u}\left(t^{i}\right)\right\|_{H^{-1}(\Omega)}^{2},\left\|\left(I-P_{i}\right) \mathbf{u}\left(t^{i}\right)\right\|_{L^{2}(\Omega)}^{2}\right)\right)
\end{aligned}
$$

where $C$ depends only on $\Omega$ and $C_{k}$ depends only on $k$. Here $\left[\mathbf{e}_{h}^{i}\right]=\mathbf{e}_{h+}^{i}-\mathbf{e}_{h-}^{i}$ is the jump in $\mathbf{e}_{h}$ at $t^{i}, \Delta t^{i} \equiv t^{i}-t^{i-1}$, and $P_{i}: L^{2}(\Omega) \rightarrow Z_{h}^{i}$ is the orthogonal projection onto the discretely divergence free space.

Proof. Substituting $\mathbf{f}(\mathbf{v})=\left\langle\mathbf{u}_{t}, \mathbf{v}\right\rangle+a(\mathbf{u}, \mathbf{v})$ into equation (4.3) gives the orthogonality relation for the total error $\mathbf{e}=\mathbf{u}-\mathbf{u}_{h}$,

$$
\left\{\begin{array}{l}
\left(\mathbf{e}_{-}^{n}, \mathbf{v}_{-}^{n}\right)+\int_{t^{n-1}}^{t^{n}}\left(-\left\langle\mathbf{e}, \mathbf{v}_{h t}\right\rangle+a\left(\mathbf{e}, \mathbf{v}_{h}\right)-b\left(\mathbf{v}_{h}, r_{h}\right)\right)-\left(\mathbf{e}_{-}^{n-1}, \mathbf{v}_{+}^{n-1}\right)=0 \\
\int_{t^{n-1}}^{t^{n}} b\left(\mathbf{u}_{h}, q_{h}\right)=0
\end{array}\right.
$$

for all $\left(\mathbf{v}_{h}, q_{h}\right) \in \mathcal{P}_{k}\left[t^{n-1}, t^{n} ; U_{h}^{n}\right] \times \mathcal{P}_{k}\left[t^{n-1}, t^{n} ; Q_{h}^{n}\right]$.

Write $\mathbf{e}=\left(\mathbf{u}-\mathbb{P}_{n}^{l o c} \mathbf{u}\right)+\left(\mathbb{P}_{n}^{l o c} \mathbf{u}-\mathbf{u}_{h}\right) \equiv \mathbf{e}_{p}+\mathbf{e}_{h}$ and note that $\mathbf{e}_{h} \in \mathcal{P}_{k}\left[t^{n-1}, t^{n} ; Z_{h}^{n}\right]$. Rearranging the orthogonality relation (4.4), and using properties of $\mathbb{P}_{n}^{l o c}$, shows

$$
\begin{aligned}
\left(\mathbf{e}_{h-}^{n}, \mathbf{v}_{-}^{n}\right)-\int_{t^{n-1}}^{t^{n}}\left(\left\langle\mathbf{e}_{h}, \mathbf{v}_{h t}\right\rangle+\right. & \left.a\left(\mathbf{e}_{h}, \mathbf{v}_{h}\right)\right)-\left(\mathbf{e}_{h-}^{n-1}, \mathbf{v}_{+}^{n-1}\right) \\
=\left(\left(I-P_{n-1}\right) \mathbf{u}\left(t^{n-1}\right), \mathbf{v}_{+}^{n-1}\right) & \\
& \quad \int_{t^{n-1}}^{t^{n}}\left(\left(\mathbf{e}_{p}, \mathbf{v}_{h t}\right)-a\left(\mathbf{e}_{p}, \mathbf{v}_{h}\right)+b\left(\mathbf{v}_{h}, r_{h}\right)\right),
\end{aligned}
$$


provided $\mathbf{v}_{-}^{n} \in Z_{h}^{n}$. Setting $\mathbf{v}_{h}=\mathbf{e}_{h}$ then shows

$$
\begin{aligned}
(1 / 2)\left\|\mathbf{e}_{h-}^{n}\right\|^{2} & +\nu C \int_{t^{n-1}}^{t^{n}}\left\|\mathbf{e}_{h}\right\|_{H^{1}(\Omega)}^{2}+(1 / 2)\left\|\left[\mathbf{e}_{h}^{n-1}\right]\right\|_{L^{2}(\Omega)}^{2} \leq(1 / 2)\left\|\mathbf{e}_{h-}^{n-1}\right\|_{L^{2}(\Omega)}^{2} \\
& +\left(\left(I-P_{n-1}\right) \mathbf{u}\left(t^{n-1}\right), \mathbf{e}_{h+}^{n-1}\right)-\int_{t^{n-1}}^{t^{n}} a\left(\mathbf{e}_{p}, \mathbf{e}_{h}\right)
\end{aligned}
$$

Here we used the fact that $\mathbf{e}_{h}(t) \in Z_{h}^{n}$ so that $b\left(\mathbf{e}_{h}, r_{h}\right)=0$, and $\mathbf{e}_{h t} \in \mathcal{P}_{k-1}\left[t^{n-1}\right.$, $\left.t^{n}, Z_{h}^{n}\right]$, so $\int_{t^{n-1}}^{t^{n}}\left(\mathbf{e}_{p}, \mathbf{e}_{h t}\right)=\int_{t^{n-1}}^{t^{n}}\left(\left(I-\mathbb{P}_{n}^{l o c}\right) \mathbf{u}, \mathbf{e}_{h t}\right)=0$. The last term on the second line is bounded using the Cauchy-Schwarz inequality, and the first term is bounded in two different ways. Since $\mathbf{e}_{h-}^{n-1} \in U_{h}^{n-1}$ an estimate independent of $\nu$ is computed as

$$
\begin{aligned}
\left(\left(I-P_{n-1}\right) \mathbf{u}\left(t^{n-1}\right), \mathbf{e}_{h+}^{n-1}\right) & =\left(\left(I-P_{n-1}\right) \mathbf{u}\left(t^{n-1}\right), \mathbf{e}_{h+}^{n-1}-\mathbf{e}_{h-}^{n-1}\right) \\
& \leq\left\|\left(I-P_{n-1}\right) \mathbf{u}\left(t^{n-1}\right)\right\|_{L^{2}(\Omega)}^{2}+(1 / 4)\left\|\left[\mathbf{e}_{h}^{n-1}\right]\right\|_{L^{2}(\Omega)}^{2} .
\end{aligned}
$$

Since $\mathbf{e}_{h+}^{n-1} \in U_{h}^{n}$, an alternative estimate is obtained by writing

$$
\begin{aligned}
\left(\left(I-P_{n-1}\right) \mathbf{u}\left(t^{n-1}\right), \mathbf{e}_{h+}^{n-1}\right) & =\left(P_{n}\left(I-P_{n-1}\right) \mathbf{u}\left(t^{n-1}\right), \mathbf{e}_{h+}^{n-1}\right) \\
& \leq\left\|P_{n}\left(I-P_{n-1}\right) \mathbf{u}\left(t^{n-1}\right)\right\|_{H^{-1}(\Omega)}\left\|\mathbf{e}_{h+}^{n-1}\right\|_{H^{1}(\Omega)} .
\end{aligned}
$$

To estimate $\left\|\mathbf{e}_{h+}^{n-1}\right\|_{H^{1}(\Omega)}$ we use the following trace estimate for functions in $\mathcal{P}_{k}\left[t^{n-1}, t^{n}, U_{h}^{n}\right]$

$$
\left\|\mathbf{e}_{h+}^{n-1}\right\|_{H^{1}(\Omega)}^{2} \leq\left(C_{k} / \Delta t^{n}\right) \int_{t^{n-1}}^{t^{n}}\left\|\mathbf{e}_{h}\right\|_{H^{1}(\Omega)}^{2} .
$$

The finite dimensionality of $\mathcal{P}_{k}\left(t^{n-1}, t^{n}\right)$, and a scaling argument, shows that the constant $C_{k}$ depends only on $k$. Therefore,

$$
\begin{aligned}
& \left(\left(I-P_{n-1}\right) \mathbf{u}\left(t^{n-1}\right), \mathbf{e}_{h+}^{n-1}\right) \\
& \quad \leq\left(C_{k} / \Delta t^{n} \nu\right)\left\|P_{n}\left(I-P_{n-1}\right) \mathbf{u}\left(t^{n-1}\right)\right\|_{H^{-1}(\Omega)}^{2}+(\nu / 4) \int_{t^{n-1}}^{t^{n}}\left\|\mathbf{e}_{h}\right\|_{H^{1}(\Omega)}^{2} .
\end{aligned}
$$

Substituting these estimates into equation (4.6) and summing completes the proof.

The previous theorem bounds the $L^{2}(\Omega)$ norm of the error at the partition points. A bootstrap argument is used next to obtain bounds at arbitrary times $t \in(0, T)$.

Theorem 4.7. Let $\mathbf{u} \in H^{1}\left[0, T ; H^{-1}(\Omega)\right] \cap L^{2}\left[0, T ; H_{0}^{1}(\Omega)\right]$ and $\mathbf{u}_{h}=\mathbb{P}_{h} \mathbf{u}$. Then the error $\mathbf{e}_{h}=\mathbb{P}_{h}^{\text {loc }} \mathbf{u}-\mathbf{u}_{h}$ satisfies

$$
\begin{gathered}
\left\|\mathbf{e}_{h}(t)\right\|_{L^{2}(\Omega)}^{2}+\nu \int_{0}^{t^{n}}\left\|\mathbf{e}_{h}\right\|_{H^{1}(\Omega)}^{2}+\sum_{i=0}^{n-1}\left\|\left[\mathbf{e}_{h}^{i}\right]\right\|_{L^{2}(\Omega)} \leq C\left(\int_{0}^{t^{n}} \nu\left\|\left(I-\mathbb{P}_{h}^{l o c}\right) \mathbf{u}\right\|_{H^{1}(\Omega)}^{2}\right. \\
\left.+\sum_{i=0}^{n-1} \min \left(1 /\left(\Delta t^{i+1} \nu\right)\left\|P_{i+1}\left(I-P_{i}\right) \mathbf{u}\left(t^{i}\right)\right\|_{H^{-1}(\Omega)}^{2},\left\|\left(I-P_{i}\right) \mathbf{u}\left(t^{i}\right)\right\|_{L^{2}(\Omega)}^{2}\right)\right),
\end{gathered}
$$

where $C$ is a constant depending only on $k$ and the domain $\Omega$. Here $\left[\mathbf{e}_{h}^{i}\right]=\mathbf{e}_{h+}^{i}-\mathbf{e}_{h-}^{i}$ is the jump in $\mathbf{e}_{h}$ at $t^{i}, \Delta t^{i} \equiv t^{i}-t^{i-1}$, and $P_{i}: L^{2}(\Omega) \rightarrow Z_{h}^{i}$ is the orthogonal projection onto the discretely divergence free space. 
Proof. Given Theorem 4.6 it suffices to bound $\left\|\mathbf{e}_{h}(t)\right\|_{L^{2}(\Omega)}$ for $t \in\left(t^{n-1}, t^{n}\right]$. Adopting the notation in the previous proof, we begin with equality (4.5)

$$
\begin{aligned}
\left(\mathbf{e}_{h-}^{n}, \mathbf{v}_{-}^{n}\right)-\int_{t^{n-1}}^{t^{n}}\left(\left\langle\mathbf{e}_{h}, \mathbf{v}_{h t}\right\rangle+a\left(\mathbf{e}_{h}, \mathbf{v}_{h}\right)\right)-\left(\mathbf{e}_{h-}^{n-1}, \mathbf{v}_{+}^{n-1}\right) \\
=\left(\left(I-P_{n-1}\right) \mathbf{u}\left(t^{n-1}\right), \mathbf{v}_{+}^{n-1}\right) \\
\quad+\int_{t^{n-1}}^{t^{n}}\left(\left(\mathbf{e}_{p}, \mathbf{v}_{h t}\right)-a\left(\mathbf{e}_{p}, \mathbf{v}_{h}\right)+b\left(\mathbf{v}_{h}, r_{h}\right)\right)
\end{aligned}
$$

and integrate parts in time to get

$$
\begin{aligned}
\int_{t^{n-1}}^{t^{n}} & \left(\left\langle\mathbf{e}_{h t}, \mathbf{v}_{h}\right\rangle+a\left(\mathbf{e}_{h}, \mathbf{v}_{h}\right)\right)+\left(\mathbf{e}_{h+}^{n-1}-\mathbf{e}_{h-}^{n-1}, \mathbf{v}_{+}^{n-1}\right) \\
= & \left(\left(I-P_{n-1}\right) \mathbf{u}\left(t^{n-1}\right), \mathbf{v}_{+}^{n-1}\right) \\
& +\int_{t^{n-1}}^{t^{n}}\left(\left(\mathbf{e}_{p}, \mathbf{v}_{h t}\right)-a\left(\mathbf{e}_{p}, \mathbf{v}_{h}\right)+b\left(\mathbf{v}_{h}, r_{h}\right)\right) .
\end{aligned}
$$

Letting $\mathbf{v}_{h}=\tilde{\mathbf{e}} \in \mathcal{P}_{k}\left[t^{n-1}, t^{n} ; Z_{h}^{n}\right]$ be the discrete approximation of $\chi_{\left[t^{n-1}, t\right)} \mathbf{e}_{h}$ characterized by equation (3.1) gives

$$
\begin{aligned}
\int_{t^{n-1}}^{t}\left(\mathbf{e}_{h t}, \tilde{\mathbf{e}}\right)+\left(\mathbf{e}_{h+}^{n-1}-\mathbf{e}_{h-}^{n-1}, \mathbf{e}_{h+}^{n-1}\right)=\left(\left(I-P_{n-1}\right) \mathbf{u}\left(t^{n-1}\right), \mathbf{e}_{h+}^{n-1}\right) \\
-\int_{t^{n-1}}^{t^{n}}\left(a\left(\mathbf{e}_{p}, \tilde{\mathbf{e}}\right)+a\left(\mathbf{e}_{h}, \tilde{\mathbf{e}}\right)\right) .
\end{aligned}
$$

As in the previous proof, $\tilde{\mathbf{e}}(t) \in Z_{h}^{n}$ so that $b\left(\tilde{\mathbf{e}}, r_{h}\right)=0$, and $\tilde{\mathbf{e}}_{t} \in \mathcal{P}_{k-1}\left[t^{n-1}, t^{n}, Z_{h}^{n}\right]$, so $\int_{t^{n-1}}^{t^{n}}\left(\mathbf{e}_{p}, \tilde{\mathbf{e}}_{t}\right)=\int_{t^{n-1}}^{t^{n}}\left(\left(I-\mathbb{P}_{n}^{l o c}\right) \mathbf{u}, \tilde{\mathbf{e}}_{t}\right)=0$. Since $\mathbf{e}_{h t} \in \mathcal{P}_{k-1}\left[t^{n-1}, t^{n}, Z_{h}^{n}\right]$ the defining property of $\tilde{\mathbf{e}}$ allows the first term to be integrated exactly:

$$
\begin{aligned}
(1 / 2) & \left\|\mathbf{e}_{h}(t)\right\|_{L^{2}(\Omega)}^{2}+(1 / 2)\left\|\left[\mathbf{e}_{h}^{n-1}\right]\right\|_{L^{2}(\Omega)}^{2}-(1 / 2)\left\|\mathbf{e}_{h-}^{n-1}\right\|_{L^{2}(\Omega)} \\
& =\left(\left(I-P_{n-1}\right) \mathbf{u}\left(t^{n-1}\right), \mathbf{e}_{h+}^{n-1}\right)-\int_{t^{n-1}}^{t^{n}}\left(a\left(\mathbf{e}_{p}, \tilde{\mathbf{e}}\right)+a\left(\mathbf{e}_{h}, \tilde{\mathbf{e}}\right)\right) .
\end{aligned}
$$

Using Lemma 3.1 to bound $\tilde{\mathbf{e}}$ in terms of $\mathbf{e}_{h}$ we obtain

$$
\begin{aligned}
& (1 / 2)\left\|\mathbf{e}_{h}(t)\right\|_{L^{2}(\Omega)}^{2}+(1 / 2)\left\|\left[\mathbf{e}_{h}^{n-1}\right]\right\|_{L^{2}(\Omega)}-(1 / 2)\left\|\mathbf{e}_{h-}^{n-1}\right\|_{L^{2}(\Omega)}^{2} \\
& \leq\left(\left(I-P_{n-1}\right) \mathbf{u}\left(t^{n-1}\right), \mathbf{e}_{h+}^{n-1}\right)+\nu C_{k} \int_{t^{n-1}}^{t^{n}}\left(\left\|\mathbf{e}_{p}\right\|_{H^{1}(\Omega)}^{2}+\left\|\mathbf{e}_{h}\right\|_{H^{1}(\Omega)}^{2}\right) .
\end{aligned}
$$

As in the proof of Theorem 4.6 the first term on the right can be bounded by

$$
\left\|\left(I-P_{n-1}\right) \mathbf{u}\left(t^{n-1}\right)\right\|_{L^{2}(\Omega)}^{2}+(1 / 4)\left\|\left[\mathbf{e}_{h}^{n-1}\right]\right\|_{L^{2}(\Omega)}^{2}
$$

or

$$
C_{k} /\left(\Delta t^{n} \nu\right)\left\|P_{n}\left(I-P_{n-1}\right) \mathbf{u}\left(t^{n-1}\right)\right\|_{H^{-1}(\Omega)}^{2}+(\nu / 4) \int_{t^{n-1}}^{t^{n}}\left\|\mathbf{e}_{h}\right\|_{H^{1}(\Omega)}^{2},
$$

with $C_{k}$ depending only on $k$. The theorem then follows by using Theorem 4.6 to bound $\left\|\mathbf{e}_{h-}^{n-1}\right\|_{L^{2}(\Omega)}$ and $\left\|\mathbf{e}_{h}\right\|_{L^{2}\left[0, T ; H^{1}(\Omega)\right]}^{2}$.

Combining the above with the triangle inequality provides various estimates for the projection error $\mathbf{u}-\mathbb{P}_{h} \mathbf{u}$ in terms of the local projection errors $\mathbf{u}-\mathbb{P}_{h}^{l o c} \mathbf{u}$. The following corollary bounds the energy and $L^{2}(\Omega)$ norms which appear in the estimates for the Navier-Stokes equation below. 
Corollary 4.8. Under the hypotheses of the theorem there exists a constant $C=$ $C(k, \Omega)$ such that

$$
\begin{aligned}
\| \mathbf{u}- & \mathbb{P}_{h} \mathbf{u}\left\|_{L^{2}\left[0, T ; H^{1}(\Omega)\right]}^{2} \leq C\right\| \mathbf{u}-\mathbb{P}_{h}^{l o c} \mathbf{u} \|_{L^{2}\left[0, T ; H^{1}(\Omega)\right]}^{2} \\
& +(C / \nu) \sum_{i=0}^{n-1} \min \left(1 /\left(\Delta t^{i+1} \nu\right)\left\|P_{i+1}\left(I-P_{i}\right) \mathbf{u}\left(t^{i}\right)\right\|_{H^{-1}(\Omega)}^{2},\left\|\left(I-P_{i}\right) \mathbf{u}\left(t^{i}\right)\right\|_{L^{2}(\Omega)}^{2}\right)
\end{aligned}
$$

and

$$
\begin{aligned}
& \left\|\mathbf{u}-\mathbb{P}_{h} \mathbf{u}\right\|_{L^{\infty}\left[0, T ; L^{2}(\Omega)\right]} \leq\left\|\mathbf{u}-\mathbb{P}_{h}^{l o c} \mathbf{u}\right\|_{L^{\infty}\left[0, T ; L^{2}(\Omega)\right]}+C \sqrt{\nu}\left\|\mathbf{u}-\mathbb{P}_{h}^{l o c} \mathbf{u}\right\|_{L^{2}\left[0, T ; H^{1}(\Omega)\right]} \\
& +C\left(\sum_{i=0}^{n-1} \min \left(1 /\left(\Delta t^{i+1} \nu\right)\left\|P_{i+1}\left(I-P_{i}\right) \mathbf{u}\left(t^{i}\right)\right\|_{H^{-1}(\Omega)}^{2},\left\|\left(I-P_{i}\right) \mathbf{u}\left(t^{i}\right)\right\|_{L^{2}(\Omega)}^{2}\right)\right)^{1 / 2} .
\end{aligned}
$$

4.3. Rates of convergence. Corollary 4.8 shows that the projection error $(I-$ $\left.\mathbb{P}_{h}\right) \mathbf{u}$ can be bounded by local projection errors of the form $\left(I-\mathbb{P}_{h}^{l o c}\right) \mathbf{u}$ and jumps in the projections at the discrete times $\left\{t^{n}\right\}$. In this section we verify that the projections $\mathbb{P}_{h} \mathbf{u}$ onto classical finite element subspaces will exhibit optimal rates of convergence when the solution $\mathbf{u}$ is sufficiently smooth.

Theorem 4.9. Assume the subspaces $\left(U_{h}^{n}, Q_{h}^{n}\right)$ satisfy the inf-sup condition and approximation and inverse properties in Assumption 1 with constants independent of $n$ and $h$, and assume that the time steps are quasi-uniform in the sense that there exists $0<\theta \leq 1$ such that $\theta \max _{1 \leq n \leq N} \Delta t^{n} \leq \min _{1 \leq n \leq N} \Delta t^{n}$. If $\mathbf{u} \in C\left[0, T ; H^{\ell+1}(\Omega) \cap V(\Omega)\right] \cap H^{k+1}\left[0, T ; H_{0}^{1}(\Omega)\right]$, then there exists a constant $C>0$ independent of $h, \tau$, and $\nu$ such that

$$
\begin{aligned}
\left\|\mathbf{u}-\mathbb{P}_{h} \mathbf{u}\right\|_{L^{2}\left[0, T ; H^{1}(\Omega)\right]} \leq & C\left(\|\mathbf{u}\|_{L^{2}\left[0, T ; H^{\ell+1}(\Omega)\right]} h^{\ell}+\left\|\mathbf{u}^{(k+1)}\right\|_{L^{2}\left[0, T ; H^{1}(\Omega)\right]} \tau^{k+1}\right. \\
& \left.+\|\mathbf{u}\|_{C\left[0, T ; H^{\ell+1}(\Omega)\right]} \min \left(h^{2} /(\tau \nu), h / \sqrt{\tau \nu}\right) h^{\ell}\right)
\end{aligned}
$$

and

$$
\begin{aligned}
\| \mathbf{u}- & \mathbb{P}_{h} \mathbf{u} \|_{L^{\infty}\left[0, T ; L^{2}(\Omega)\right]} \leq C\left(\left(\sqrt{\nu}\|\mathbf{u}\|_{L^{2}\left[0, T ; H^{\ell+1}(\Omega)\right]}+h\|\mathbf{u}\|_{L^{\infty}\left[0, T ; H^{\ell+1}(\Omega)\right]}\right) h^{\ell}\right. \\
& +\left(\sqrt{\nu}\left\|\mathbf{u}^{(k+1)}\right\|_{L^{2}\left[0, T ; H^{1}(\Omega)\right]}+\left\|\mathbf{u}^{(k+1)}\right\|_{L^{2}\left[0, T ; L^{2}(\Omega)\right]}\right) \tau^{k+1} \\
& \left.+\|\mathbf{u}\|_{C\left[0, T ; H^{\ell+1}(\Omega)\right]} \min \left(h^{3 / 2} /(\tau \sqrt{\nu}), \sqrt{h / \tau}\right) h^{\ell+1 / 2}\right) .
\end{aligned}
$$

where $\tau=\max _{1 \leq n \leq N} \Delta t^{n}$, and $\mathbf{u}^{(k+1)}$ denotes the $(k+1)$ st time derivative of $\mathbf{u}$.

Proof. Theorem 4.3 and the approximation properties of the projections $P_{n}$ given in Corollary 4.5 show

$$
\begin{aligned}
\| \mathbf{u}- & \mathbb{P}_{n}^{l o c} \mathbf{u}\left\|_{L^{2}\left[t^{n-1}, t^{n} ; H^{1}(\Omega)\right]} \leq C\right\| \mathbf{u}-P_{n} \mathbf{u} \|_{L^{2}\left[t^{n-1}, t^{n} ; H^{1}(\Omega)\right]} \\
& +C\left(\Delta t^{n}\right)^{k+1}\left\|\mathbf{u}^{(k+1)}\right\|_{L^{2}\left[t^{n-1}, t^{n} ; H^{1}(\Omega)\right]} \\
\leq & C\|\mathbf{u}\|_{L^{2}\left[t^{n-1}, t^{n} ; H^{\ell+1}(\Omega]\right.} h^{\ell}+C \tau^{k+1}\left\|\mathbf{u}^{(k+1)}\right\|_{L^{2}\left[t^{n-1}, t^{n} ; H^{1}(\Omega)\right]},
\end{aligned}
$$

which shows

$$
\left\|\mathbf{u}-\mathbb{P}_{h}^{l o c} \mathbf{u}\right\|_{L^{2}\left[0, T ; H^{1}(\Omega)\right]} \leq C\left(\|\mathbf{u}\|_{L^{2}\left[0, T ; H^{\ell+1}(\Omega)\right]} h^{\ell}+\left\|\mathbf{u}^{(k+1)}\right\|_{L^{2}\left[0, T ; H^{1}(\Omega)\right]} \tau^{k+1}\right) .
$$


The estimates in Corollary 4.5 are used to estimate the jump terms.

$$
\begin{gathered}
\sum_{i=0}^{n-1} \min \left(1 /\left(\Delta t^{i+1} \nu\right)\left\|P_{i+1}\left(I-P_{i}\right) \mathbf{u}\left(t^{i}\right)\right\|_{H^{-1}(\Omega)}^{2},\left\|\left(I-P_{i}\right) \mathbf{u}\left(t^{i}\right)\right\|_{L^{2}(\Omega)}^{2}\right) \\
\leq C \sum_{i=0}^{n-1} \min \left(\left(1 / \Delta t^{i+1} \nu\right) h^{2(\ell+2)}, h^{2(\ell+1)}\right)\left\|\mathbf{u}\left(t^{i}\right)\right\|_{H^{\ell+1}(\Omega)}^{2} \\
\leq C(T, \theta)\|\mathbf{u}\|_{C\left[0, T ; H^{\ell+1}(\Omega)\right]}^{2} \min \left(h^{4} /\left(\tau^{2} \nu\right), h^{2} / \tau\right) h^{2 \ell}
\end{gathered}
$$

Combining these two estimates with the bound in Corollary 4.8 establishes the bound in $L^{2}\left[0, T ; H^{1}(\Omega)\right]$.

The first estimate of Theorem 4.3 shows

$$
\begin{aligned}
\| \mathbf{u}- & \mathbb{P}_{n}^{l o c} \mathbf{u}\left\|_{L^{\infty}\left[t^{n-1}, t^{n} ; L^{2}(\Omega)\right]} \leq\right\| \mathbf{u}-P_{n} \mathbf{u} \|_{L^{\infty}\left[t^{n-1}, t^{n} ; L^{2}(\Omega)\right]} \\
& +C_{k}\left(\Delta t^{i}\right)^{k+1}\left\|\mathbf{u}^{(k+1)}\right\|_{L^{\infty}\left[t^{n-1}, t^{n} ; L^{2}(\Omega)\right]} \\
\leq & C\|\mathbf{u}\|_{L^{\infty}\left[t^{n-1}, t^{n} ; H^{\ell+1}(\Omega]\right.} h^{\ell+1}+C \tau^{k+1}\left\|\mathbf{u}^{(k+1)}\right\|_{L^{\infty}\left[t^{n-1}, t^{n} ; L^{2}(\Omega)\right]} .
\end{aligned}
$$

Combining this with the above establishes the second statement of the theorem.

4.4. Stability in $L^{\infty}\left[0, T ; H^{1}(\Omega)\right]$. For the nonlinear problem, we will need a bound on $\left\|\mathbb{P}_{h} \mathbf{u}\right\|_{L^{\infty}\left[0, T ; H^{1}(\Omega)\right]}$ when $\mathbf{u}_{t}-\nu \Delta \mathbf{u} \in L^{2}\left[0, T ; L^{2}(\Omega)\right]$. That is, discontinuous Galerkin approximations of Stokes problem are bounded in $L^{\infty}\left[0, T ; H^{1}(\Omega)\right]$ when $\mathbf{f} \in L^{2}[0, T ; W(\Omega)]$ and $\mathbf{u}_{0} \in V(\Omega)$. The following proof uses the exponential interpolant of Definition 3.3 .

Theorem 4.10. Let $\mathbf{u}_{h}$ be a solution of the discontinuous Galerkin scheme (4.3) with data $\mathbf{f} \equiv \mathbf{u}_{t}-\nu \Delta \mathbf{u} \in L^{2}[0, T ; W(\Omega)]$ and $\mathbf{u}_{0} \in V(\Omega)$. Then,

$$
\left\|\mathbf{u}_{h}\right\|_{L^{\infty}\left[0, T ; H^{1}(\Omega)\right]} \leq C\left(\left\|\mathbf{u}_{0}\right\|_{H^{1}(\Omega)}+(1 / \sqrt{\nu})\|\mathbf{f}\|_{L^{2}\left[0, T ; L^{2}(\Omega)\right]}\right)
$$

where $C$ depends only on $k$ and the domain $\Omega$.

Proof. We construct a discrete approximation of the $\Delta \mathbf{u}_{h}$. At each time $t \in$ $\left[t^{n-1}, t^{n}\right)$ let $\mathbf{a}_{p} \in Z_{h}^{n}$ be the discrete approximation of $\Delta \mathbf{u}_{h}$ satisfying

$$
\left(\mathbf{a}_{p}, \mathbf{w}_{h}\right)=(1 / \nu) a\left(\mathbf{u}_{h}, \mathbf{w}_{h}\right), \quad \forall \mathbf{w}_{h} \in Z_{h}^{n} .
$$

Then $\mathbf{a}_{p} \in \mathcal{P}_{k}\left[t^{n-1}, t^{n} ; Z_{h}^{n}\right]$, and setting $\mathbf{w}_{h}()=.\mathbf{u}_{h t}(.) \in Z_{h}^{n}$ and $\mathbf{w}_{h}()=.\mathbf{a}_{p}(.) \in$ $Z_{h}^{n}$ respectively, we obtain

$$
\begin{aligned}
(1 / 2) \frac{d}{d t}\left\|\nabla \mathbf{u}_{h}\right\|_{L^{2}(\Omega)}^{2} & =(1 / \nu) a\left(\mathbf{u}_{h}, \mathbf{u}_{h t}\right)=\left(\mathbf{a}_{p}, \mathbf{u}_{h t}\right) \text { and } \\
a\left(\mathbf{u}_{h}, \mathbf{a}_{p}\right) & =\nu\left\|\mathbf{a}_{p}\right\|_{L^{2}(\Omega)}^{2} .
\end{aligned}
$$

Setting $\mathbf{v}_{h}=\mathbf{a}_{p}$ into equation (4.3), and using the last two equalities, gives

$$
\begin{aligned}
& (1 / 2)\left\|\nabla \mathbf{u}_{h-}^{n}\right\|_{L^{2}(\Omega)}^{2}+(1 / 2)\left\|\left[\nabla \mathbf{u}_{h}^{n-1}\right]\right\|_{H^{1}(\Omega)}^{2}+\nu \int_{t^{n-1}}^{t^{n}}\left\|\mathbf{a}_{p}\right\|_{L^{2}(\Omega)}^{2} \\
& =(1 / 2)\left\|\nabla \mathbf{u}_{h-}^{n-1}\right\|_{L^{2}(\Omega)}^{2}+\int_{t^{n-1}}^{t^{n}}\left(\mathbf{f}, \mathbf{a}_{p}\right) .
\end{aligned}
$$


Using Cauchy-Schwarz and Young inequalities for the last term we obtain the bound

$$
\begin{aligned}
\left\|\nabla \mathbf{u}_{h-}^{n}\right\|_{L^{2}(\Omega)}^{2}+ & \left\|\left[\nabla \mathbf{u}_{h}^{n-1}\right]\right\|_{L^{2}(\Omega)}^{2}+(\nu / 2) \int_{t^{n-1}}^{t^{n}}\left\|\mathbf{a}_{p}\right\|_{L^{2}(\Omega)}^{2} \\
& \leq\left\|\nabla \mathbf{u}_{h-}^{n-1}\right\|_{L^{2}(\Omega)}^{2}+(1 / \nu) \int_{t^{n-1}}^{t^{n}}\|\mathbf{f}\|_{L^{2}(\Omega)}^{2} .
\end{aligned}
$$

This inequality implies the desired bound at partition points, and for the low order schemes, $k=0$ and $k=1$, also bounds $\left\|\mathbf{u}_{h}\right\|_{L^{\infty}\left[0, T ; H^{1}(\Omega)\right]}$. For higher order schemes, we obtain an additional estimate using the discrete approximation of $\mathbf{a}_{p} e^{-\lambda\left(t-t^{n-1}\right)}$. Let $\overline{\mathbf{a}}_{p} \in \mathcal{P}_{k}\left[t^{n-1}, t^{n} ; Z_{h}^{n}\right]$ be the discrete approximation of $\mathbf{a}_{p} e^{-\lambda\left(t-t^{n-1}\right)}$ constructed in Definition 3.3 . Then

$\int_{t^{n-1}}^{t^{n}}\left(\mathbf{u}_{h t}, \overline{\mathbf{a}}_{p}\right)=\int_{t^{n-1}}^{t^{n}}\left(\mathbf{u}_{h t}, \mathbf{a}_{p}\right) e^{-\lambda\left(t-t^{n-1}\right)}=(1 / 2) \int_{t^{n-1}}^{t^{n}} e^{-\lambda\left(t-t^{n-1}\right)} \frac{d}{d t}\left\|\nabla \mathbf{u}_{h}\right\|_{L^{2}(\Omega)}^{2}$,

where at the last equality used (4.7). Integrating equation (4.3) by parts, setting $\mathbf{v}_{h}=\overline{\mathbf{a}}_{p}$, using the definition of $\mathbf{a}_{p}$, and Lemma 3.6, gives

$$
\begin{aligned}
(1 / 2) \| & \nabla \mathbf{u}_{h-}^{n}\left\|_{L^{2}(\Omega)}^{2} e^{-\lambda\left(t^{n}-t^{n-1}\right)}+(1 / 2)\right\|\left[\nabla \mathbf{u}_{h}^{n-1}\right] \|_{L^{2}(\Omega)}^{2} \\
& +\int_{t^{n-1}}^{t^{n}}(\lambda / 2) e^{-\lambda\left(t-t^{n-1}\right)}\left\|\nabla \mathbf{u}_{h}\right\|_{L^{2}(\Omega)}^{2} \\
\leq & (1 / 2)\left\|\nabla \mathbf{u}_{h-}^{n-1}\right\|_{L^{2}(\Omega)}^{2}+\int_{t^{n-1}}^{t^{n}}\left|\left(\mathbf{f}, \overline{\mathbf{a}}_{p}\right)\right|+\left|a\left(\mathbf{u}_{h}, \overline{\mathbf{a}}_{p}\right)\right| \\
\leq & (1 / 2)\left\|\nabla \mathbf{u}_{h-}^{n-1}\right\|_{L^{2}(\Omega)}^{2}+\int_{t^{n-1}}^{t^{n}}(1 / 4 \nu)\|\mathbf{f}\|_{L^{2}(\Omega)}^{2}+\nu\left\|\mathbf{a}_{p}\right\|_{L^{2}(\Omega)}^{2}+\nu\left(\mathbf{a}_{p}, \overline{\mathbf{a}}_{p}\right) \\
\leq & (1 / 2)\left\|\nabla \mathbf{u}_{h-}^{n-1}\right\|_{L^{2}(\Omega)}^{2}+\int_{t^{n-1}}^{t^{n}}(1 / 4 \nu)\|\mathbf{f}\|_{L^{2}(\Omega)}^{2}+\left(1+C_{k}\right) \nu\left\|\mathbf{a}_{p}\right\|_{L^{2}(\Omega)}^{2} .
\end{aligned}
$$

Using equation (4.8) to bound the terms on the right, it follows that

$$
\int_{t^{n-1}}^{t^{n}} \lambda e^{-\lambda\left(t-t^{n-1}\right)}\left\|\nabla \mathbf{u}_{h}\right\|_{L^{2}(\Omega)}^{2} \leq C\left(\left\|\mathbf{u}_{h}^{0}\right\|_{H^{1}(\Omega)}^{2}+(1 / \nu) \int_{0}^{t^{n}}\|\mathbf{f}\|_{L^{2}(\Omega)}^{2}\right)
$$

where $C$ depends on domain and $k$ (but not on $\lambda$ ). Selecting $\lambda=1 /\left(t^{n}-t^{n-1}\right.$ ) shows

$$
\int_{t^{n-1}}^{t^{n}} e^{-1} /\left(t^{n}-t^{n-1}\right)\left\|\nabla \mathbf{u}_{h}\right\|_{L^{2}(\Omega)}^{2} \leq C\left(\left\|\mathbf{u}_{h}^{0}\right\|_{H^{1}(\Omega)}^{2}+(1 / \nu) \int_{0}^{t^{n}}\|f\|_{L^{2}(\Omega)}^{2}\right) .
$$

Since $\nabla \mathbf{u}_{h} \in \mathcal{P}_{k}\left[t^{n-1}, t^{n} ; L^{2}(\Omega)\right]$, Lemma 3.5 (with weight $w(t) \equiv 1$ and $p=\infty$ ) states

$$
\left\|\nabla \mathbf{u}_{h}\right\|_{L^{\infty}\left[t^{n-1}, t^{n} ; L^{2}(\Omega)\right]}^{2} \leq\left(C_{k} /\left(t^{n}-t^{n-1}\right)\right)\left\|\nabla \mathbf{u}_{h}\right\|_{L^{2}\left[t^{n-1}, t^{n} ; L^{2}(\Omega)\right]}^{2},
$$

and the theorem follows.

4.5. Estimates for the Stokes problem. In this section we sketch how the arguments used in this section also provide error estimates for DG approximations of the Stokes problem. These results are of independent interest and are not used below. 
The orthogonality condition for (4.3) takes the form

$$
\left\{\begin{array}{l}
\left(\mathbf{e}_{-}^{n}, \mathbf{v}_{-}^{n}\right)+\int_{t^{n-1}}^{t^{n}}\left(-\left\langle\mathbf{e}, \mathbf{v}_{h t}\right\rangle+a\left(\mathbf{e}, \mathbf{v}_{h}\right)+b\left(\mathbf{v}_{h}, p-p_{h}\right)\right)-\left(\mathbf{e}_{-}^{n-1}, \mathbf{v}_{+}^{n-1}\right)=0, \\
\int_{t^{n-1}}^{t^{n}} b\left(\mathbf{e}, q_{h}\right)=0
\end{array}\right.
$$

for all $\left(\mathbf{v}_{h}, q_{h}\right) \in \mathcal{P}_{k}\left[t^{n-1}, t^{n} ; U_{h}^{n}\right] \times \mathcal{P}_{k}\left[t^{n-1}, t^{n} ; Q_{h}^{n}\right]$. Here, $\mathbf{e}=\mathbf{u}-\mathbf{u}_{h},\left(\mathbf{u}_{h}, p_{h}\right)$ is the solution of (4.3), and $(\mathbf{u}, p) \in L^{2}[0, T ; V(\Omega)] \cap H^{1}\left[0, T ; H^{-1}(\Omega)\right] \times L^{2}\left[0, T ; L_{0}^{2}(\Omega)\right]$ is the solution of the parabolic Stokes problem.

Estimates for $\mathbf{e}$ were obtained by selecting $\mathbf{v}_{h} \in \mathcal{P}_{k}\left[t^{n-1}, t^{n} ; Z_{h}^{n}\right]$ which eliminates the discrete pressure; $\int_{t^{n-1}}^{t^{n}} b\left(\mathbf{v}_{h}, p-p_{h}\right)=\int_{t^{n-1}}^{t^{n}} b\left(\mathbf{v}_{h}, p-q_{h}\right)$, for all $q_{h} \in$ $L^{2}\left[t^{n-1}, t^{n} ; Q_{h}^{n}\right]$. In this situation the proof of Theorem 4.7 shows that the error $\mathbf{e}_{h}=\mathbb{P}_{h}^{l o c} \mathbf{u}-\mathbf{u}_{h}$ satisfies

$$
\begin{aligned}
&\left\|\mathbf{e}_{h}(t)\right\|_{L^{2}(\Omega)}^{2}+\nu \int_{0}^{t^{n}}\left\|\mathbf{e}_{h}\right\|_{H^{1}(\Omega)}^{2}+\sum_{i=0}^{n-1}\left\|\left[\mathbf{e}_{h}^{i}\right]\right\|_{L^{2}(\Omega)} \\
& \leq C\left(\left\|\mathbf{e}_{h}^{0}\right\|_{L^{2}(\Omega)}^{2}+\int_{0}^{t^{n}} \nu\left\|\left(I-\mathbb{P}_{h}^{l o c}\right) \mathbf{u}\right\|_{H^{1}(\Omega)}^{2}+(1 / \nu) \int_{0}^{t^{n}}\left\|p-q_{h}\right\|_{L^{2}(\Omega)}^{2}\right. \\
&\left.+\sum_{i=0}^{n-1} \min \left(1 /\left(\Delta t^{i+1} \nu\right)\left\|P_{i+1}\left(I-P_{i}\right) \mathbf{u}\left(t^{i}\right)\right\|_{H^{-1}(\Omega)}^{2},\left\|\left(I-P_{i}\right) \mathbf{u}\left(t^{i}\right)\right\|_{L^{2}(\Omega)}^{2}\right)\right),
\end{aligned}
$$

where $C$ is a constant depending only on $k$ and the domain $\Omega$, and $q_{h}$ is arbitrary.

Rates of convergence then follow as in Theorem 4.9

$$
\begin{aligned}
\| \mathbf{u}- & \mathbb{P}_{h} \mathbf{u} \|_{L^{2}\left[0, T ; H^{1}(\Omega)\right]} \leq C\left(\|\mathbf{u}\|_{L^{2}\left[0, T ; H^{\ell+1}(\Omega)\right]} h^{\ell}+\left\|\mathbf{u}^{(k+1)}\right\|_{L^{2}\left[0, T ; H^{1}(\Omega)\right]} \tau^{k+1}\right. \\
& +(1 / \sqrt{\nu})\|\mathbf{u}(0)\|_{H^{\ell}(\Omega)} h^{\ell} \\
& \left.+(1 / \nu)\|p\|_{L^{2}\left[0, T ; H^{\ell}(\Omega)\right]} h^{\ell}+\|\mathbf{u}\|_{C\left[0, T ; H^{\ell+1}(\Omega)\right]} \min \left(h^{2} /(\tau \nu), h / \sqrt{\tau \nu}\right) h^{\ell}\right)
\end{aligned}
$$

and

$$
\begin{aligned}
\| \mathbf{u} & -\mathbb{P}_{h} \mathbf{u} \|_{L^{\infty}\left[0, T ; L^{2}(\Omega)\right]} \leq C\left(\left(\sqrt{\nu}\|\mathbf{u}\|_{L^{2}\left[0, T ; H^{\ell+1}(\Omega)\right]}+h\|\mathbf{u}\|_{L^{\infty}\left[0, T ; H^{\ell+1}(\Omega)\right]}\right) h^{\ell}\right. \\
& +\left(\sqrt{\nu}\left\|\mathbf{u}^{(k+1)}\right\|_{L^{2}\left[0, T ; H^{1}(\Omega)\right]}+\left\|\mathbf{u}^{(k+1)}\right\|_{L^{\infty}\left[0, T ; L^{2}(\Omega)\right]}\right) \tau^{k+1}+\|\mathbf{u}(0)\|_{H^{\ell}(\Omega)} h^{\ell} \\
& \left.+\|\mathbf{u}\|_{C\left[0, T ; H^{\ell+1}(\Omega)\right]} \min \left(h^{3 / 2} /(\tau \sqrt{\nu}), \sqrt{h / \tau}\right) h^{\ell+1 / 2}+(1 / \sqrt{\nu})\|p\|_{L^{2}\left[0, T ; H^{\ell}(\Omega)\right]} h^{\ell}\right) .
\end{aligned}
$$

where $\tau=\max _{1 \leq n \leq N} \Delta t^{n}$.

\section{Analysis of the DG SCheme for the NavieR-Stokes Equations}

5.1. Stability. The natural energy estimate for the discontinuous Galerkin scheme (2.4) is obtained by setting $\mathbf{v}_{h}=\mathbf{u}_{h}$ and using the skew symmetry of the trilinear form, $c\left(\mathbf{u}_{h}, \mathbf{u}_{h}, \mathbf{u}_{h}\right)=0$, to get

$$
\begin{array}{r}
(1 / 2)\left\|\mathbf{u}_{-}^{n}\right\|_{L^{2}(\Omega)}^{2}+(1 / 2)\left\|\left[\mathbf{u}^{n-1}\right]\right\|_{L^{2}(\Omega)}^{2}+\nu \int_{t^{n-1}}^{t^{n}}\left\|\mathbf{u}_{h}\right\|_{H^{1}(\Omega)}^{2} \\
\leq(1 / 2)\left\|\mathbf{u}_{-}^{n-1}\right\|_{L^{2}(\Omega)}^{2}+\int_{t^{n-1}}^{t^{n}}\left\langle\mathbf{f}, \mathbf{u}_{h}\right\rangle .
\end{array}
$$


In general it is necessary to bound the last term on the right by

$$
\left\langle\mathbf{f}, \mathbf{u}_{h}\right\rangle \leq\|\mathbf{f}\|_{H^{-1}(\Omega)}\left\|\mathbf{u}_{h}\right\|_{H^{1}(\Omega)} \leq(1 / 2 \nu)\|\mathbf{f}\|_{H^{-1}(\Omega)}^{2}+(\nu / 2)\left\|\mathbf{u}_{h}\right\|_{H^{1}(\Omega)}^{2},
$$

which gives rise to a factor of $1 / \nu$ on the right-hand side.

The implicit Euler scheme $(k=0)$ has $\mathbf{u}_{h}$ is piecewise constant in time, so $\mathbf{u}_{h}=\mathbf{u}_{-}^{n}$ on $\left(t^{n-1}, t^{n}\right)$ and the Cauchy-Schwarz inequality can be used to bound the right-hand side of equation (5.1). In this situation

$$
\begin{aligned}
& \left(1-\Delta t^{n}\right)\left\|\mathbf{u}_{-}^{n}\right\|_{L^{2}(\Omega)}^{2}+\left\|\left[\mathbf{u}^{n-1}\right]\right\|_{L^{2}(\Omega)}^{2}+2 \nu \int_{t^{n-1}}^{t^{n}}\left\|\mathbf{u}_{h}\right\|_{H^{1}(\Omega)}^{2} \\
& \leq\left\|\mathbf{u}_{-}^{n-1}\right\|_{L^{2}(\Omega)}^{2}+\int_{t^{n-1}}^{t^{n}}\|\mathbf{f}\|_{L^{2}(\Omega)}^{2},
\end{aligned}
$$

which gives bounds independent of $\nu$. Similarly, if $\mathbf{u}_{h}$ is piecewise linear, an explicit computation shows

$$
\int_{t^{n-1}}^{t^{n}}\left\|\mathbf{u}_{h}\right\|_{L^{2}(\Omega)}^{2} \leq \hat{C} \Delta t^{n}\left(\left\|\mathbf{u}_{-}^{n}\right\|_{L^{2}(\Omega)}^{2}+\left\|\mathbf{u}_{-}^{n-1}\right\|_{L^{2}(\Omega)}^{2}+\left\|\left[\mathbf{u}^{n-1}\right]\right\|_{L^{2}(\Omega)}^{2}\right),
$$

where $\hat{C}=(6+\sqrt{6}) / 4$. It follows that

$$
\begin{gathered}
\left(1-\hat{C} \Delta t^{n}\right)\left\|\mathbf{u}_{-}^{n}\right\|_{L^{2}(\Omega)}^{2}+c\left\|\left[\mathbf{u}^{n-1}\right]\right\|_{L^{2}(\Omega)}^{2}+2 \nu \int_{t^{n-1}}^{t^{n}}\left\|\mathbf{u}_{h}\right\|_{H^{1}(\Omega)}^{2} \\
\leq\left(1+\hat{C} \Delta t^{n}\right)\left\|\mathbf{u}_{-}^{n-1}\right\|_{L^{2}(\Omega)}^{2}+\int_{t^{n-1}}^{t^{n}}\|\mathbf{f}\|_{L^{2}(\Omega)}^{2},
\end{gathered}
$$

and if $\hat{C} \Delta t^{n}<1$, the discrete Gronwall inequality is applicable. These observations are summarized in the following lemma.

Lemma 5.1. Let $\left(\mathbf{u}_{h}, p_{h}\right)$ be an approximate solution of the Navier-Stokes equations computed using the discontinuous Galerkin scheme (2.4) with $\mathbf{f} \in L^{2}[0, T$; $\left.H^{-1}(\Omega)\right]$ and $\mathbf{u}_{0} \in L^{2}(\Omega)$. Then

$$
\left\|\mathbf{u}_{-}^{n}\right\|_{L^{2}(\Omega)}^{2}+\nu \int_{0}^{t^{n}}\left\|\mathbf{u}_{h}\right\|_{H^{1}(\Omega)}^{2} \leq\left\|\mathbf{u}_{-}^{0}\right\|_{L^{2}(\Omega)}^{2}+(C / \nu) \int_{0}^{t^{n}}\|\mathbf{f}\|_{H^{-1}(\Omega)}^{2}
$$

where $C$ depends only on the domain $\Omega$. Moreover, when $\mathbf{f} \in L^{2}\left[0, T ; L^{2}(\Omega)\right]$, the low order $D G$ schemes, $k=0$ and $k=1$ also satisfy

$$
\left\|\mathbf{u}_{-}^{n}\right\|_{L^{2}(\Omega)}^{2}+\nu \int_{0}^{t^{n}}\left\|\mathbf{u}_{h}\right\|_{H^{1}(\Omega)}^{2} \leq C e^{\hat{C} t^{n}}\left(\left\|\mathbf{u}_{-}^{0}\right\|_{L^{2}(\Omega)}^{2}+\int_{0}^{t^{n}}\|\mathbf{f}\|_{L^{2}(\Omega)}^{2}\right),
$$

provided the maximal time step $\tau=\max _{1 \leq n \leq N} \Delta t^{n}$ is smaller than some absolute constant $\hat{C}$.

5.2. Error estimates for the Navier-Stokes Equations. Since solutions $(\mathbf{u}, p)$ of the Navier-Stokes equations satisfy the Discontinuous Galerkin formulation (2.4) the error $\mathbf{e}=\mathbf{u}-\mathbf{u}_{h}$ satisfies the orthogonality relation,

$$
\left\{\begin{array}{c}
\left(\mathbf{e}_{-}^{n}, \mathbf{v}_{-}^{n}\right)+\int_{t^{n-1}}^{t^{n}}\left(-\left(\mathbf{e}, \mathbf{v}_{h t}\right)+a\left(\mathbf{e}, \mathbf{v}_{h}\right)+b\left(\mathbf{v}_{h}, p-p_{h}\right)\right)-\left(\mathbf{e}_{-}^{n-1}, \mathbf{v}_{+}^{n-1}\right) \\
\quad=-\int_{t^{n-1}}^{t^{n}}\left(c\left(\mathbf{u}, \mathbf{u}, \mathbf{v}_{h}\right)-c\left(\mathbf{u}_{h}, \mathbf{u}_{h}, \mathbf{v}_{h}\right)\right) \quad \forall \mathbf{v}_{h} \in \mathcal{P}_{k}\left[t^{n-1}, t^{n} ; U_{h}^{n}\right] \\
\int_{t^{n-1}}^{t^{n}} b\left(\mathbf{e}, q_{h}\right)=0, \quad \forall q_{h} \in \mathcal{P}_{k}\left[t^{n-1}, t^{n} ; Q_{h}^{n}\right]
\end{array}\right.
$$


Our main theorem, stated next, is valid for both two- and three-dimensional domains and schemes of arbitrary order in space and time.

Theorem 5.2. Let $d=2$ or 3 and let $(\mathbf{u}, p)$ be a solution of the Navier-Stokes equations with $\mathbf{u} \in L^{\infty}\left[0, T ; H^{1}(\Omega)\right]$. Let $\left(\mathbf{u}_{h}, p_{h}\right)$ be an approximate solution of $(\mathbf{u}, p)$ computed using the discontinuous Galerkin scheme (2.4), and write the error in the velocity as $\mathbf{e}=\mathbf{e}_{p}+\mathbf{e}_{h}$, with $\mathbf{e}_{p}=\mathbf{u}-\mathbb{P}_{h} \mathbf{u}$ and $\mathbf{e}_{h}=\mathbb{P}_{h} \mathbf{u}-\mathbf{u}_{h}$, where $\mathbb{P}_{h} \mathbf{u}$ is the projection defined in Definition 4.2 ,

Then there exists a constant $C=C(k, T, \Omega)$ such that

$$
\begin{aligned}
& \left\|\mathbf{e}_{h-}^{n}\right\|_{L^{2}(\Omega)}^{2}+\sum_{i=0}^{n-1}\left\|\left[\mathbf{e}_{h}^{i}\right]\right\|_{L^{2}(\Omega)}^{2}+\nu \int_{0}^{t^{n}}\left\|\mathbf{e}_{h}\right\|_{H^{1}(\Omega)}^{2} \leq(C / \nu) \exp (C T)\left\{\left\|\mathbf{e}_{h-}^{0}\right\|^{2}\right. \\
& \left.\quad+\int_{0}^{t^{n}}\left(\left(\|\mathbf{u}\|_{L^{\infty}\left[0, T ; H^{1}(\Omega)\right]}^{2}+\left\|\mathbb{P}_{h} \mathbf{u}\right\|_{L^{\infty}\left[0, T ; H^{1}(\Omega)\right]}^{2}\right)\left\|\mathbf{e}_{p}\right\|_{H^{1}(\Omega)}^{2}+\left\|p-q_{h}\right\|_{L^{2}(\Omega)}^{2}\right)\right\},
\end{aligned}
$$

for any $q_{h} \in\left\{L^{2}\left[0, T ; L^{2}(\Omega)\right]\left|q_{h}\right|_{\left(t^{n-1}, t^{n}\right)} \in L^{2}\left[t^{n-1}, t^{n} ; Q_{h}^{n}\right]\right\}$ provided the maximum time step size $\tau=\max _{1 \leq n \leq N}\left(t^{n}-t^{n-1}\right)$ is sufficiently small. In particular, for the low order schemes, $k=0$ and $k=1$, we require $C\left\|\mathbb{P}_{h} \mathbf{u}\right\|_{L^{\infty}\left[0, T ; H^{1}(\Omega)\right]}^{4} \tau / \nu^{3}<1$, and, additionally,

$$
C\left(1 / \nu+\left\|\mathbb{P}_{h} \mathbf{u}\right\|_{L^{\infty}\left[0, T ; H^{1}(\Omega)\right]} \sqrt{\tau}\right)\left\|\mathbb{P}_{h} \mathbf{u}\right\|_{L^{\infty}\left[0, T ; H^{1}(\Omega)\right]}^{4} \sqrt{\tau} / \nu^{4}<1
$$

for the higher order schemes, $k \geq 2$.

Recall that, if $\mathbf{u}_{t}-\Delta \mathbf{u} \in L^{2}\left[0, T ; L^{2}(\Omega)\right]$, then $\mathbf{u} \in L^{\infty}\left[0, T ; H^{1}(\Omega)\right]$ and Theorem 4.10 shows that this is sufficient to guarantee that $\mathbb{P}_{h} \mathbf{u} \in L^{\infty}\left[0, T ; H^{1}(\Omega)\right]$ too. Also, note that the DG approximation scheme is stable for any time step size $\tau>0$, and that the restriction on $\tau$ and $L^{\infty}\left[0, T ; H^{1}(\Omega)\right]$ bounds assumed in the theorem are only necessary in order to quantify the error.

Proof. 1) Orthogonality: Introducing the splitting $\mathbf{e}=\mathbf{e}_{p}+\mathbf{e}_{h}$ into the orthogonality relation (5.2) gives

$$
\begin{aligned}
\int_{t^{n-1}}^{t^{n}} & \left(\left(\mathbf{e}_{h t}, \mathbf{v}_{h}\right)+a\left(\mathbf{e}_{h}, \mathbf{v}_{h}\right)\right)+\left(\mathbf{e}_{h+}^{n-1}-\mathbf{e}_{h-}^{n-1}, \mathbf{v}_{h+}^{n-1}\right) \\
= & -\int_{t^{n-1}}^{t^{n}}\left(c\left(\mathbf{u}, \mathbf{u}, \mathbf{v}_{h}\right)-c\left(\mathbf{u}_{h}, \mathbf{u}_{h}, \mathbf{v}_{h}\right)-b\left(\mathbf{v}_{h}, p-p_{h}\right)\right) \\
& -\int_{t^{n-1}}^{t^{n}}\left(\left(\mathbf{e}_{p t}, \mathbf{v}_{h}\right)+a\left(\mathbf{e}_{p}, \mathbf{v}_{h}\right)\right)-\left(\mathbf{e}_{p+}^{n-1}-\mathbf{e}_{p-}^{n-1}, \mathbf{v}_{h+}^{n-1}\right)
\end{aligned}
$$

for all $\mathbf{v}_{h} \in \mathcal{P}_{k}\left[t^{n-1}, t^{n} ; Q_{h}^{n}\right]$. By construction, $\mathbf{e}_{p}$ satisfies the orthogonality condition (4.4), which, after integration by parts in time, states

$$
\int_{t^{n-1}}^{t^{n}}\left(\left(\mathbf{e}_{p t}, \mathbf{v}_{h}\right)+a\left(\mathbf{e}_{p}, \mathbf{v}_{h}\right)+b\left(\mathbf{v}_{h}, r_{h}\right)\right)+\left(\mathbf{e}_{p+}^{n-1}-\mathbf{e}_{p-}^{n-1}, \mathbf{v}_{h+}^{n-1}\right)=0 .
$$

Combining the last two equations, we obtain

$$
\begin{aligned}
\int_{t^{n-1}}^{t^{n}} & \left(\left(\mathbf{e}_{h t}, \mathbf{v}_{h}\right)+a\left(\mathbf{e}_{h}, \mathbf{v}_{h}\right)\right)+\left(\mathbf{e}_{h+}^{n-1}-\mathbf{e}_{h-}^{n-1}, \mathbf{v}_{h+}^{n-1}\right) \\
= & \int_{t^{n-1}}^{t^{n}}\left(c\left(\mathbf{u}, \mathbf{u}, \mathbf{v}_{h}\right)-c\left(\mathbf{u}_{h}, \mathbf{u}_{h}, \mathbf{v}_{h}\right)-b\left(\mathbf{v}_{h}, p-p_{h}-r_{h}\right)\right) .
\end{aligned}
$$


We will always select $\mathbf{v}_{h} \in \mathcal{P}_{k}\left[t^{n-1}, t^{n} ; Z_{h}^{n}\right]$ so that the pressure term becomes

$$
\int_{t^{n-1}}^{t^{n}} b\left(\mathbf{v}_{h}, p-p_{h}-r_{h}\right)=\int_{t^{n-1}}^{t^{n}} b\left(\mathbf{v}_{h}, p\right)=\int_{t^{n-1}}^{t^{n}} b\left(\mathbf{v}_{h}, p-q_{h}\right) .
$$

In this equality we may choose $q_{h} \in L^{2}\left[t^{n-1}, t^{n} ; Q_{h}^{n}\right]$, i.e., $q_{h}$ does not need to have polynomial time dependence. Letting $\mathbf{u}_{p}=\mathbb{P}_{h} \mathbf{u}$, algebraic manipulation using trilinearity of $c(., .,$.$) shows$

$$
\begin{aligned}
& c\left(\mathbf{u}, \mathbf{u}, \mathbf{v}_{h}\right)-c\left(\mathbf{u}_{h}, \mathbf{u}_{h}, \mathbf{v}_{h}\right) \\
& \quad=c\left(\mathbf{e}_{p}, \mathbf{u}, \mathbf{v}_{h}\right)+c\left(\mathbf{u}_{p}, \mathbf{e}_{p}, \mathbf{v}_{h}\right)+c\left(\mathbf{e}_{h}, \mathbf{u}_{p}, \mathbf{v}_{h}\right)+c\left(\mathbf{u}_{h}, \mathbf{e}_{h}, \mathbf{v}_{h}\right) .
\end{aligned}
$$

It remains to bound each term on the right when $\mathbf{v}_{h}=\mathbf{e}_{h}$.

2) Bound the Nonlinear Terms: The skew symmetry properties and bounds of Lemma 2.2 show that $c\left(\mathbf{u}_{h}, \mathbf{e}_{h}, \mathbf{e}_{h}\right)=0$ and

$$
\begin{aligned}
& \int_{t^{n-1}}^{t^{n}}\left|c\left(\mathbf{e}_{p}, \mathbf{u}, \mathbf{e}_{h}\right)\right| \leq C \int_{t^{n-1}}^{t^{n}}\left\|\mathbf{e}_{p}\right\|_{H^{1}(\Omega)}\|\mathbf{u}\|_{H^{1}(\Omega)}\left\|\mathbf{e}_{h}\right\|_{H^{1}(\Omega)} \\
& \leq(C / \epsilon)\|\mathbf{u}\|_{L^{\infty}\left[0, T ; H^{1}(\Omega)\right]}^{2} \int_{t^{n-1}}^{t^{n}}\left\|\mathbf{e}_{p}\right\|_{H^{1}(\Omega)}^{2}+\epsilon \int_{t^{n-1}}^{t^{n}}\left\|\mathbf{e}_{h}\right\|_{H^{1}(\Omega)}^{2} \\
& \int_{t^{n-1}}^{t^{n}}\left|c\left(\mathbf{u}_{p}, \mathbf{e}_{p}, \mathbf{e}_{h}\right)\right| \leq C \int_{t^{n-1}}^{t^{n}}\left\|\mathbf{u}_{p}\right\|_{H^{1}(\Omega)}\left\|\mathbf{e}_{p}\right\|_{H^{1}(\Omega)}\left\|\mathbf{e}_{h}\right\|_{H^{1}(\Omega)} \\
& \leq(C / \epsilon)\left\|\mathbf{u}_{p}\right\|_{L^{\infty}\left[0, T ; H^{1}(\Omega)\right]}^{2} \int_{t^{n-1}}^{t^{n}}\left\|\mathbf{e}_{p}\right\|_{H^{1}(\Omega)}^{2}+\epsilon \int_{t^{n-1}}^{t^{n}}\left\|\mathbf{e}_{h}\right\|_{H^{1}(\Omega)}^{2} \\
& \int_{t^{n-1}}^{t^{n}}\left|c\left(\mathbf{e}_{h}, \mathbf{u}_{p}, \mathbf{e}_{h}\right)\right| \leq C \int_{t^{n-1}}^{t^{n}}\left\|\mathbf{e}_{h}\right\|_{L^{2}(\Omega)}^{1 / 2}\left\|\mathbf{e}_{h}\right\|_{H^{1}(\Omega)}^{1 / 2}\left\|\mathbf{u}_{p}\right\|_{H^{1}(\Omega)}\left\|\mathbf{e}_{h}\right\|_{H^{1}(\Omega)} \\
& \leq\left(C / \epsilon^{3}\right)\left\|\mathbf{u}_{p}\right\|_{L^{\infty}\left[0, T ; H^{1}(\Omega)\right]}^{4} \int_{t^{n-1}}^{t^{n}}\left\|\mathbf{e}_{h}\right\|_{L^{2}(\Omega)}^{2}+\epsilon \int_{t^{n-1}}^{t^{n}}\left\|\mathbf{e}_{h}\right\|_{H^{1}(\Omega)}^{2}
\end{aligned}
$$

where $C$ denotes a constant that depends only on $\Omega$. Setting $\mathbf{v}_{h}=\mathbf{e}_{h}$ in equation (5.3) and collecting the above bounds with $\epsilon=O(\nu)$ we obtain

$$
\begin{aligned}
& \left\|\mathbf{e}_{h-}^{n}\right\|_{L^{2}(\Omega)}^{2}+\left\|\left[\mathbf{e}_{h}^{n-1}\right]\right\|_{L^{2}(\Omega)}^{2}+\int_{t^{n-1}}^{t^{n}}(\nu / 2)\left\|\mathbf{e}_{h}\right\|_{H^{1}(\Omega)}^{2} \leq\left\|\mathbf{e}_{h-}^{n-1}\right\|_{L^{2}(\Omega)}^{2} \\
& \quad+\left(C_{1}^{4} / \nu^{3}\right) \int_{t^{n-1}}^{t^{n}}\left\|\mathbf{e}_{h}\right\|_{L^{2}(\Omega)}^{2}+(C / \nu) \int_{t^{n-1}}^{t^{n}}\left(C_{2}^{2}\left\|\mathbf{e}_{p}\right\|_{H^{1}(\Omega)}^{2}+\left\|p-q_{h}\right\|_{L^{2}(\Omega)}^{2}\right),
\end{aligned}
$$

where $C=C(\Omega)$ is independent of $\nu$ and $C_{1}$ and $C_{2}$ take the form

$$
\begin{aligned}
& C_{1}=C\left\|\mathbf{u}_{p}\right\|_{L^{\infty}\left[0, T ; H^{1}(\Omega)\right]}, \\
& C_{2}=C\left(\|\mathbf{u}\|_{L^{\infty}\left[0, T ; H^{1}(\Omega)\right]}+\left\|\mathbf{u}_{p}\right\|_{L^{\infty}\left[0, T ; H^{1}(\Omega)\right]}\right) .
\end{aligned}
$$

For the low order schemes, $k=0$ and $k=1,\left\|\mathbf{e}_{h}\right\|_{L^{2}(\Omega)}$ is bounded by $\mathbf{e}_{-}^{n}, \mathbf{e}_{-}^{n-1}$ and $\left[\mathbf{e}^{n-1}\right]$ as in the discussion preceding Lemma 5.1. An application of Gronwall's inequality then completes the proof. For the higher order schemes it is necessary to bound $\int_{t^{n-1}}^{t^{n}}\left\|\mathbf{e}_{h}\right\|_{L^{2}(\Omega)}^{2}$. 
3) Bounding $\left\|\mathbf{e}_{h}\right\|_{L^{2}(\Omega)}$ : Fix $\mathbf{z}_{h} \in Z_{h}^{n}$ and $t \in\left(t^{n-1}, t^{n}\right)$ and select $\mathbf{v}_{h}(s)=$ $\phi(s) \mathbf{z}_{h}$ where $\mathbf{v}_{h} \in \mathcal{Z}_{h}^{n}$ and $\phi \in \mathcal{P}_{k}\left(t^{n-1}, t^{n}\right)$ satisfies

$$
\phi\left(t^{n-1}\right)=1, \quad \int_{t^{n-1}}^{t^{n}} \phi \psi=\int_{t^{n-1}}^{t} \psi, \quad \psi \in \mathcal{P}_{k-1}\left(t^{n-1}, t^{n}\right) .
$$

(That is, $\mathbf{v}_{h}$ is the discrete approximation of $\chi_{\left[t^{n-1}, t\right)} \mathbf{z}_{h}$ constructed in Section 3.1). Recall that Lemma 3.2 shows that $\|\phi\|_{L^{\infty}\left(t^{n-1}, t^{n}\right)} \leq C_{k}$ where $C_{k}$ is a constant independent of $t$. Since $\mathbf{e}_{h t} \in \mathcal{P}_{k-1}\left[t^{n-1}, t^{n} ; Z_{h}^{n}\right]$ it follows that

$$
\int_{t^{n-1}}^{t^{n}}\left(\mathbf{e}_{h t}, \mathbf{v}_{h}\right)=\int_{t^{n-1}}^{t}\left(\mathbf{e}_{h t}, \mathbf{z}_{h}\right)=\left(\mathbf{e}_{h}(t)-\mathbf{e}_{h+}^{n-1}, \mathbf{z}_{h}\right) .
$$

With this choice of $\mathbf{v}_{h}$ equation (5.3) becomes

$$
\left(\mathbf{e}_{h}(t)-\mathbf{e}_{h-}^{n-1}, \mathbf{z}_{h}\right)=\int_{t^{n-1}}^{t^{n}}\left(-a\left(\mathbf{e}_{h}, \mathbf{z}_{h}\right)+c\left(\mathbf{u}_{h}, \mathbf{u}_{h}, \mathbf{z}_{h}\right)-c\left(\mathbf{u}, \mathbf{u}, \mathbf{z}_{h}\right)-b\left(\mathbf{z}_{h}, p-q_{h}\right)\right) \phi .
$$

Splitting the trilinear terms as in equation (5.4) we obtain

$$
\begin{aligned}
\left(\mathbf{e}_{h}(t)-\right. & \left.\mathbf{e}_{h-}^{n-1}, \mathbf{z}_{h}\right) \leq C \int_{t^{n-1}}^{t^{n}}\left(\nu\left\|\mathbf{e}_{h}\right\|_{H^{1}(\Omega)}+\left\|\mathbf{e}_{p}\right\|_{H^{1}(\Omega)}\|\mathbf{u}\|_{H^{1}(\Omega)}\right. \\
& +\left\|\mathbf{u}_{p}\right\|_{H^{1}(\Omega)}\left\|\mathbf{e}_{p}\right\|_{H^{1}(\Omega)}+\left\|\mathbf{e}_{h}\right\|_{H^{1}(\Omega)}\left\|\mathbf{u}_{p}\right\|_{H^{1}(\Omega)} \\
& \left.\quad+\left\|\mathbf{u}_{h}\right\|_{H^{1}(\Omega)}\left\|\mathbf{e}_{h}\right\|_{H^{1}(\Omega)}+\left\|p-q_{h}\right\|_{L^{2}(\Omega)}\right)\left\|\mathbf{z}_{h}\right\|_{H^{1}(\Omega)} \\
\leq & C\left(\left(\nu+\left\|\mathbf{u}_{p}\right\|_{L^{\infty}\left[t^{n-1}, t^{n} ; H^{1}(\Omega)\right]}\right) \tau^{1 / 2}\left\|\mathbf{e}_{h}\right\|_{L^{2}\left[t^{n-1}, t^{n} ; H^{1}(\Omega)\right]}\right. \\
& +\left(\|\mathbf{u}\|_{L^{\infty}\left[t^{n-1}, t^{n} ; H^{1}(\Omega)\right]}+\left\|\mathbf{u}_{p}\right\|_{L^{\infty}\left[t^{n-1}, t^{n} ; H^{1}(\Omega)\right]}\right) \tau^{1 / 2}\left\|\mathbf{e}_{p}\right\|_{L^{2}\left[t^{n-1}, t^{n} ; H^{1}(\Omega)\right]} \\
& +\left\|\mathbf{u}_{h}\right\|_{L^{2}\left[0, T ; H^{1}(\Omega)\right]}\left\|\mathbf{e}_{h}\right\|_{L^{2}\left[t^{n-1}, t^{n} ; H^{1}(\Omega)\right]} \\
& \left.+\tau^{1 / 2}\left\|p-q_{h}\right\|_{L^{2}\left[t^{n-1}, t^{n} ; L^{2}(\Omega)\right]}\right)\left\|\mathbf{z}_{h}\right\|_{H^{1}(\Omega)} \\
\leq & C\left(\left(\nu+C_{1}\right) \sqrt{\tau}\left\|\mathbf{e}_{h}\right\|_{L^{2}\left[t^{n-1}, t^{n} ; H^{1}(\Omega)\right]}+C_{2} \sqrt{\tau}\left\|\mathbf{e}_{p}\right\|_{L^{2}\left[t^{n-1}, t^{n} ; H^{1}(\Omega)\right]}\right. \\
& \left.+(1 / \nu)\left\|\mathbf{e}_{h}\right\|_{L^{2}\left[t^{n-1}, t^{n} ; H^{1}(\Omega)\right]}+\tau^{1 / 2}\left\|p-q_{h}\right\|_{L^{2}\left[t^{n-1}, t^{n} ; L^{2}(\Omega)\right]}\right)\left\|\mathbf{z}_{h}\right\|_{H^{1}(\Omega)}
\end{aligned}
$$

where the constant $C=(\Omega)$ is independent of $\nu$ and $C_{1}, C_{2}$ depend on $\mathbf{u}$ as in equation (5.6). Now note that $\nu+C_{1} \sim C_{1}$, select $\mathbf{z}_{h}=\mathbf{e}_{h}(t)$ and integrate with respect to $t$ to get,

$$
\begin{aligned}
& \int_{t^{n-1}}^{t^{n}}\left(\mathbf{e}_{h}(t)-\mathbf{e}_{h-}^{n-1}, \mathbf{e}_{h}(t)\right) \leq C\left(C_{1} \sqrt{\tau}\left\|\mathbf{e}_{h}\right\|_{L^{2}\left[t^{n-1}, t^{n} ; H^{1}(\Omega)\right]}+C_{2} \sqrt{\tau}\left\|\mathbf{e}_{p}\right\|_{L^{2}\left[t^{n-1}, t^{n} ; H^{1}(\Omega)\right]}\right. \\
& \left.\quad+(1 / \nu)\left\|\mathbf{e}_{h}\right\|_{L^{2}\left[t^{n-1}, t^{n} ; H^{1}(\Omega)\right]}+\tau^{1 / 2}\left\|p-q_{h}\right\|_{L^{2}\left[t^{n-1}, t^{n} ; L^{2}(\Omega)\right]}\right) \int_{t^{n-1}}^{t^{n}}\left\|\mathbf{e}_{h}\right\|_{H^{1}(\Omega)} .
\end{aligned}
$$

Therefore, with $\Delta t^{n}=t^{n}-t^{n-1}$ we have

$$
\begin{aligned}
& \int_{t^{n-1}}^{t^{n}}\left\|\mathbf{e}_{h}\right\|_{L^{2}(\Omega)}^{2} \leq \Delta t^{n}\left\|\mathbf{e}_{h}^{n-1}\right\|_{L^{2}(\Omega)}^{2} \\
& \quad+C \sqrt{\tau} \int_{t^{n-1}}^{t^{n}}\left(\left(1 / \nu+C_{1} \sqrt{\tau}\right)\left\|\mathbf{e}_{h}\right\|_{H^{1}(\Omega)}^{2}+\left(C_{2} \sqrt{\tau}\right)\left\|\mathbf{e}_{p}\right\|_{H^{1}(\Omega)}^{2}\right) \\
& \quad+\sqrt{\tau}\left\|p-q_{h}\right\|_{L^{2}(\Omega)}^{2} .
\end{aligned}
$$


4) Combining the Estimates: Using inequality (5.7) to bound the integral of $\left\|\mathbf{e}_{h}\right\|_{L^{2}(\Omega)}^{2}$ on the right-hand side of equation (5.5) we obtain

$$
\begin{aligned}
\left\|\mathbf{e}_{h-}^{n}\right\|_{L^{2}(\Omega)}^{2}+\|\left[\mathbf{e}_{h}^{n-1}\left\|_{L^{2}(\Omega)}^{2}+\int_{t^{n-1}}^{t^{n}}\left(\nu / 2-\left(1 / \nu+C_{1} \sqrt{\tau}\right) C_{1}^{4} \sqrt{\tau} / \nu^{3}\right)\right\| \mathbf{e}_{h} \|_{H^{1}(\Omega)}^{2}\right. \\
\leq\left(1+C_{1}^{4} \Delta t^{n} / \nu^{3}\right)\left\|\mathbf{e}_{h-}^{n-1}\right\|_{L^{2}(\Omega)}^{2}+C \int_{t^{n-1}}^{t^{n}}\left(C_{2} / \nu+C_{1}^{4} \tau / \nu^{3}\right) C_{2}\left\|\mathbf{e}_{p}\right\|_{H^{1}(\Omega)}^{2} \\
\quad+\left(1 / \nu+C_{1}^{4} \tau / \nu^{3}\right)\left\|p-q_{h}\right\|_{L^{2}(\Omega)}^{2} .
\end{aligned}
$$

Under the assumptions on the maximal time step $\tau$, application of the discrete Gronwall Lemma completes the proof.

The technical development in Step 3 of our proof was necessary since the discrete energy estimate does not naturally bound the $L^{2}(\Omega)$ norm of the solution at times between the partition points. In Appendix $\mathrm{A}$ it is shown that in two dimensions it is possible to bound the discrete solution $\mathbf{u}_{h}$ in $L^{\infty}\left[0, T ; L^{2}(\Omega)\right]$.

Theorem 4.9 can now be used to establish rates of convergence for smooth solutions.

Theorem 5.3. Let $\Omega \subset \mathbb{R}^{d}$ with $d=2$ or 3 and for $h>0$ let $\left\{t_{h}^{n}\right\}_{n=0}^{N_{h}}$ be a partition of $[0, T]$ and let $\left\{\left(U_{h}^{n}, Q_{h}^{n}\right)\right\}_{n=0}^{N_{h}}$ be finite element subspaces of $H_{0}^{1}(\Omega)^{d} \times L^{2}(\Omega) / \mathbb{R}$. Assume that the time partition is quasi-uniform in the sense that there exists $0<$ $\theta \leq 1$ such that $\theta \max _{1 \leq n \leq N} \Delta t_{h}^{n} \leq \theta \min _{1 \leq n \leq N} \Delta t_{h}^{n}$, and that the finite element subspaces satisfy the approximation, inf-sup, and inverse hypotheses in Assumption 1.

Let $\left\{\left(\mathbf{u}_{h}, p_{h}\right)\right\}_{h>0}$ be approximate solutions of the Navier-Stokes equations computed using the discontinuous Galerkin scheme (2.4) and let the solution (u,p) of the Navier-Stokes equations satisfy

$$
\mathbf{u} \in C\left[0, T ; H^{\ell+1}(\Omega)\right] \cap H^{k+1}\left[0, T ; L^{2}(\Omega)\right] \quad \text { and } \quad p \in L^{2}\left[0, T ; H^{\ell}(\Omega)\right],
$$

where $\ell \geq 1$ and $k \geq 0$ are the polynomial degrees of the space and time dependence of $\left(\mathbf{u}_{h}, p_{h}\right)$.

If the initial data $\mathbf{u}_{-}^{0}$ satisfies $\left\|\mathbf{u}_{-}^{0}-P_{0} \mathbf{u}(0)\right\|_{L^{2}(\Omega)} \leq C h^{\ell}\|\mathbf{u}(0)\|_{H^{\ell}(\Omega)}$, there exists constant $C=C(k, T, \Omega)>0$ independent of $0<\nu \leq 1$ such that the error $\mathbf{e}=\mathbf{u}-\mathbf{u}_{h}$ satisfies

$$
\begin{aligned}
\left\|\mathbf{e}_{-}^{n}\right\|_{L^{2}(\Omega)} & +\sqrt{\nu}\|\mathbf{e}\|_{L^{2}\left[0, T ; H^{1}(\Omega)\right]} \leq C\left(\exp \left(C T / \nu^{3}\right)\right)^{1 / 2}\left\{1 / \sqrt{\nu}\left\|\mathbf{u}_{-}^{0}\right\|_{H^{\ell}(\Omega)} h^{\ell}\right. \\
& +\left(C_{2} / \sqrt{\nu}\right)\left(\|\mathbf{u}\|_{L^{2}\left[0, T ; H^{\ell+1}(\Omega)\right]} h^{\ell}+\left\|\mathbf{u}^{(k+1)}\right\|_{L^{2}\left[0, T ; H^{1}(\Omega)\right]} \tau^{k+1}\right. \\
& \left.\left.+\|\mathbf{u}\|_{C\left[0, T ; H^{\ell+1}(\Omega)\right]} \min \left\{\frac{h^{2}}{\tau \nu}, \frac{h}{\sqrt{\tau \nu}}\right\} h^{\ell}\right)+(1 / \sqrt{\nu})\|p\|_{L^{2}\left[0, T ; H^{\ell}(\Omega)\right]} h^{\ell}\right\},
\end{aligned}
$$

provided the maximum time step size $\tau=\max _{1 \leq n \leq N_{h}}\left(t_{h}^{n}-t_{h}^{n-1}\right)$ is sufficiently small. In particular, for the low order schemes, $k=0$ and $k=1$, we require $C\left\|\mathbf{u}_{p}\right\|_{L^{\infty}\left[0, T ; H^{1}(\Omega)\right]}^{4} \tau / \nu^{3}<1$, and additionally,

$$
C\left(1 / \nu+\left\|\mathbf{u}_{p}\right\|_{L^{\infty}\left[0, T ; H^{1}(\Omega)\right]} \sqrt{\tau}\right)\left\|\mathbf{u}_{p}\right\|_{L^{\infty}\left[0, T ; H^{1}(\Omega)\right]}^{4} \sqrt{\tau} / \nu^{4}<1
$$

for the higher order schemes, $k \geq 2$. Here

$$
C_{2}=C\left(\|\mathbf{u}\|_{L^{\infty}\left[0, T ; H^{1}(\Omega)\right]}+\left\|\mathbf{u}_{p}\right\|_{L^{\infty}\left[0, T ; H^{1}(\Omega)\right]}\right), \mathbf{u}^{(k+1)}
$$


denotes the $(k+1)$ st time derivative of $\mathbf{u}$, and $\mathbf{u}_{p} \equiv \mathbb{P}_{h} \mathbf{u}$ is the projection defined in Definition 4.2.

5.3. Comments on regularity. While the DG scheme for the Navier-Stokes equations will converge under minimal regularity guaranteed by the energy estimate (see e.g. [37), in order to obtain rates of convergence we assume restrictions on the time step which depend on $\nu,\|\mathbf{u}\|_{L^{\infty}\left[0, T ; H^{1}(\Omega)\right]}$ and $\left\|\mathbb{P}_{h} \mathbf{u}\right\|_{L^{\infty}\left[0, T ; H^{1}(\Omega)\right]}$. Using Theorem 4.10, the latter two norms can be bounded by

$$
C\left(\left\|\mathbf{u}_{0}\right\|_{H^{1}(\Omega)}+(1 / \sqrt{\nu})\left\|\mathbf{u}_{t}-\nu \Delta \mathbf{u}\right\|_{L^{2}\left[0, T ; L^{2}(\Omega)\right]}\right) .
$$

Here we briefly recall bounds for this term provided by the existence theory for solutions of the Navier-Stokes equations. Comprehensive surveys of various regularity results for the Navier-Stokes equations can be found in [12, 31, 32.

For the two-dimensional case, the regularity theorems in [12, 31 show that if $\mathbf{f} \in L^{2}[0, T ; W(\Omega)]$ and $\mathbf{u}_{0} \in V(\Omega)$, then the unique solution $(\mathbf{u}, p)$ of equations (2.1) satisfies

$$
(\mathbf{u}, p) \in L^{2}\left[0, T ; H^{2}(\Omega) \cap V(\Omega)\right] \cap H^{1}[0, T ; W(\Omega)] \times L^{2}\left[0, T ; H^{1}(\Omega) \cap L_{0}^{2}(\Omega)\right] .
$$

The time-step restriction in Theorem 5.2 depends on $\|\mathbf{u}\|_{L^{\infty}\left[0, T ; H^{1}(\Omega)\right]}$ which may be arbitrarily large for arbitrary data $\mathbf{f}, \mathbf{u}_{0}, T>0$ and $\nu$. In this situation our theorem only guarantees that asymptotic rates will be observed for very small time steps.

In the three-dimensional case, the situation is delicate. There are two distinct cases:

Small data and large times. The fundamental existence theorem in [12, Theorem 9.3, pp 80] states that for small initial data and forcing term there exists a strong solution for any time interval $[0, T]$. The next theorem summarizes relevant bounds upon the solution available from the results in [12.

Theorem 5.4. Let $\Omega$ be an open bounded set in $\mathbb{R}^{3}$ of class $C^{2}$. There exists a positive constant $C$ depending only upon $\Omega$, such that for $\mathbf{u} \in V(\Omega)$, and $\mathbf{f} \in$ $L^{2}[0, T ; W(\Omega)]$ satisfying

$$
\left\|\mathbf{u}_{0}\right\|_{H^{1}(\Omega)}^{2}+(1 / \nu)\|\mathbf{f}\|_{L^{2}\left[0, T ; L^{2}(\Omega)\right]}^{2} \leq C \nu^{2},
$$

there exists a strong solution $\mathbf{u}$ of Navier-Stokes equations belonging to

$$
L^{\infty}[0, T ; V(\Omega)] \cap L^{2}\left[0, T ; H^{2}(\Omega) \cap V(\Omega)\right]
$$

and satisfying

$$
\|\mathbf{u}\|_{L^{\infty}\left[0, T ; H^{1}(\Omega)\right]}^{2}+\nu\|\mathbf{u}\|_{L^{2}\left[0, T ; H^{2}(\Omega)\right]}^{2} \leq C \nu^{2} .
$$

In addition, $\left\|\mathbf{u}_{t}\right\|_{L^{2}\left[0, T ; L^{2}(\Omega)\right]}^{2} \leq C \nu^{3}+\mathcal{O}\left(\nu^{4}\right)$.

Under the assumption of this theorem, Theorem 4.10 shows that

$$
\left\|\mathbf{u}_{p}\right\|_{L^{\infty}\left[0, T ; H^{1}(\Omega)\right]} \leq C\left(\left\|\mathbf{u}_{0}\right\|_{H^{1}(\Omega)}+(1 / \sqrt{\nu})\left\|\mathbf{u}_{t}-\nu \Delta \mathbf{u}\right\|_{L^{2}\left[0, T ; L^{2}(\Omega)\right]}\right) \leq C \nu
$$

(assuming $\nu<1$ ). Theorem 5.2 then shows that asymptotic rates of convergence for these solutions will be observed when $C \tau<1 / \nu$ for low order schemes $(k=0$ and $k=1)$ and

$$
C(k)(1 / \nu+\nu \sqrt{\tau}) \sqrt{\tau}<1, \quad \text { which implies } \quad \tau \leq C(k) \nu^{2}
$$

for the higher order schemes. 
Large data and small times. We consider the case where $\nu<\left\|\mathbf{u}_{0}\right\|_{H^{1}(\Omega)}$ with $\nu$ small. The following existence and regularity theorem from [12, Theorem 9.4] shows that there exists a strong (hence unique) solution for a short time.

Theorem 5.5. Let $\Omega$ be an open bounded set in $\mathbb{R}^{3}$ of class $C^{2}$. There exists a positive constant $C$, depending on $\Omega$ such that for $\mathbf{u} \in V(\Omega)$, and $\mathbf{f} \in L^{2}\left[0, T_{0} ; W(\Omega)\right]$ satisfying

$$
\frac{1}{\nu^{3}}\|\mathbf{f}\|_{L^{2}\left[0, T_{0} ; L^{2}(\Omega)\right]}^{2}+\nu T_{0} \leq \frac{C}{\left(1+\frac{\left\|\mathbf{u}_{0}\right\|_{H^{1}(\Omega)}^{2}}{\nu^{2}}\right)^{2}},
$$

there exists a solution $\mathbf{u} \in L^{\infty}\left[0, T_{0} ; V(\Omega)\right] \cap L^{2}\left[0, T_{0} ; H^{2}(\Omega) \cap V(\Omega)\right]$, such that

$$
\|\mathbf{u}\|_{L^{\infty}\left[0, T_{0} ; H^{1}(\Omega)\right]}^{2}+(\nu / 2)\|\mathbf{u}\|_{L^{2}\left[0, T_{0} ; H^{2}(\Omega)\right]}^{2} \leq C\left\|\mathbf{u}_{0}\right\|_{H^{1}(\Omega)}^{2} .
$$

In addition,

$$
\left\|\mathbf{u}_{t}\right\|_{L^{2}\left[0, T ; L^{2}(\Omega)\right]}^{2} \leq C\left(\nu\left\|\mathbf{u}_{0}\right\|_{H^{1}(\Omega)}^{2}+\nu^{3}\left\|\mathbf{u}_{0}\right\|_{H^{1}(\Omega)}^{2}\right) .
$$

Under the assumption of this theorem, Theorem 4.10 shows that

$$
\begin{array}{r}
\left\|\mathbf{u}_{p}\right\|_{L^{\infty}\left[0, T_{0} ; H^{1}(\Omega)\right]} \leq C(k)\left(\left\|\mathbf{u}_{0}\right\|_{H^{1}(\Omega)}+(1 / \sqrt{\nu})\left\|\mathbf{u}_{t}\right\|_{L^{2}\left[0, T_{0} ; L^{2}(\Omega)\right.}\right. \\
\left.+\sqrt{\nu}\|\mathbf{u}\|_{L^{2}\left[0, T_{0} ; H^{2}(\Omega)\right]}\right) \\
\leq C(k)\left(\left\|\mathbf{u}_{0}\right\|_{H^{1}(\Omega)}+\sqrt{\nu}\left\|\mathbf{u}_{0}\right\|_{H^{1}(\Omega)}\right) \approx C(k)\left\|\mathbf{u}_{0}\right\|_{H^{1}(\Omega)},
\end{array}
$$

for small $\nu<1$ and arbitrary $\mathbf{u}_{0}$ with $\mathbf{f}, T_{0}, \nu$ satisfying condition (5.9). Theorem 5.2 then shows that asymptotic rates of convergence for these solutions will be observed for low order schemes $(k=0$ and $k=1)$ when $\tau \leq C \nu^{3}$, for low order schemes, where the constant $C$ also depends on the size of $\left\|\mathbf{u}_{0}\right\|_{H^{1}(\Omega)}$. Since $T_{0} \sim O\left(\nu^{3}\right)$ it follows that $\tau / T_{0} \sim O(1)$. For higher order schemes the time-step restriction of Theorem 5.2 takes the form:

$$
C(k)\left(1 / \nu+\left\|\mathbf{u}_{0}\right\|_{H^{1}(\Omega)} \sqrt{\tau}\right)\left\|\mathbf{u}_{0}\right\|_{H^{1}(\Omega)}^{4} \sqrt{\tau} / \nu^{4}<1 .
$$

Remark 1. Inspection of the proof of Theorem 5.2, reveals that the term $(1 / \nu)$ in the first term on the left came from the term $\|\mathbf{f}\|_{L^{2}\left[0, T ; H^{-1}(\Omega)\right]} / \nu$ used to bound $\left\|\mathbf{u}_{h}\right\|_{L^{2}\left[0, T_{0} ; H^{1}(\Omega)\right]}$ in Section 5.1. In the current context this term is small, so the time-step restriction for the higher order schemes becomes

$$
C(k)(1+\sqrt{\tau})\left\|\mathbf{u}_{0}\right\|_{H^{1}(\Omega)}^{5} \sqrt{\tau} / \nu^{4}<1, \quad \text { so } \quad \tau / T_{0} \leq C \nu^{5} .
$$

\section{Numerical illustration}

We present some numerical examples which illustrate the conclusions of Theorem 5.3. Exact solutions of the Navier-Stokes equations used for benchmarking may be found in [16, 34, 35]. These solutions are qualitatively similar, and the following solution of the homogeneous problem due to Shapiro [29] was selected to benchmark 
the DG scheme. Writing $\mathbf{u}=(u, v, w)$,

$$
\begin{aligned}
u= & -\frac{1}{k^{2}+\ell^{2}}(\lambda \ell \cos (k x) \sin (\ell y) \sin (m z) \\
& +m k \sin (k x) \cos (\ell y) \cos (m z)) \exp \left(-\nu \lambda^{2} t / 2\right), \\
v= & \frac{1}{k^{2}+\ell^{2}}(\lambda k \sin (k x) \cos (\ell y) \sin (m z) \\
& -m \ell \cos (k x) \sin (\ell y) \cos (m z)) \exp \left(-\nu \lambda^{2} t / 2\right), \\
w= & \cos (k x) \cos (\ell y) \sin (m z) \exp \left(-\nu \lambda^{2} t / 2\right) \\
p= & -\left(u^{2}+v^{2}+w^{2}\right) / 2
\end{aligned}
$$

where $\lambda^{2}=k^{2}+\ell^{2}+m^{2}$ and $k, \ell, m$ are specified. Taylor's two-dimensional solution is obtained by letting $\ell \rightarrow 0$ (Taylor vortices). Writing $\mathbf{u}=(u, w)$ and $\mathbf{x}=(x, z)$,

$$
\begin{aligned}
u & =(-m / k) \sin (k x) \cos (m z) \exp \left(-\nu \lambda^{2} t / 2\right), \\
v & =(\lambda / k) \sin (k x) \sin (m z) \exp \left(-\nu \lambda^{2} t / 2\right), \\
w & =\cos (k x) \sin (m z) \exp \left(-\nu \lambda^{2} t / 2\right), \\
p & =-\left(u^{2}+v^{2}+w^{2}\right) / 2,
\end{aligned}
$$

where $\lambda^{2}=k^{2}+m^{2}$ and $v$ becomes an auxiliary variable used to calculate the pressure.

For our numerical examples we set

$$
\Omega=[-1,1]^{d}, \quad \nu=1 / 20, \quad k=\pi, \quad \ell=3 \pi / 2, \quad m=\pi / 2,
$$

and Neumann boundary conditions on the top $(y=1$ in $2 \mathrm{~d}$, and $z=1$ in $3 \mathrm{~d})$, and Dirichlet data on the remainder of the boundary. Recall that when the skew symmetric form of the nonlinear term is used, the Neumann condition specifies

$$
\mathbf{t}=(-p I+\nu D(\mathbf{u})) \mathbf{n}+(1 / 2)(\mathbf{u} . \mathbf{n}) \mathbf{u}
$$

on the boundary. Approximate solutions were computed using the Taylor-Hood $Q_{2} / Q_{1}$ element for the spatial approximation of the velocity/pressure on quadrilateral $(2 \mathrm{~d})$ or hexahedral $(3 \mathrm{~d})$ subdivisions of $[-1,1]^{d}$, and polynomials of degree $0 \leq k \leq 3$ were used for the temporal variation.

For a fixed mesh, our theorems state that (for smooth solutions) the $L^{2}[0, T$; $H^{1}(\Omega)$ ] norm of the velocity error is of order $O\left(h^{2}+\tau^{k+1}\right)$, and this rate is observed for the $2 \mathrm{~d}$ solution in Figures 6.1 and 6.4 where $h / 2=\tau$ with $k=1$ and $\tau=(h / 2)^{1 / 2}$ with $k=3$, respectively, and for the 3d solution in Figure 6.3 where $h / 2=\tau$ and $k=1$. This later example illustrates that, at least for smooth solutions, restrictions on the time steps assumed in Theorem [5.3 are not always necessary in order to realize asymptotic rates of convergence for high order DG time stepping.

Errors are also tabulated for the $L^{2}\left[0, T ; L^{2}(\Omega)\right]$ norm of the pressure and velocity for which optimal rates are $O\left(h^{2}+\tau^{k+1}\right)$ and $O\left(h^{3}+\tau^{k+1}\right)$, respectively. The numerical results indicate that, for this example, the spatial errors dominate the temporal error since rate of convergence for the velocity in $L^{2}\left[0, T ; L^{2}(\Omega)\right]$ is observed to be $O\left(h^{3}\right)$ when $k=1$. To quantify this, one can compute the piecewise linear interpolants of $\exp \left(-\nu \lambda^{2} t / 2\right)$ on $[0,1]$. For a partition with 128 intervals the $L^{2}(\Omega)$ error is $4.578751 \mathrm{e}-07$ which is smaller than the errors in Figure 6.1 . 


\begin{tabular}{|c|ccc|ccc|}
\hline$h / 2=\tau$ & $\left\|e_{p}(1)\right\|_{L^{2}(\Omega)}$ & $\left\|\mathbf{e}_{u}(1)\right\|_{L^{2}(\Omega)}$ & $\left\|\mathbf{e}_{u}(1)\right\|_{H_{0}^{1}(\Omega)}$ & $\left\|e_{p}\right\|_{L^{2}\left[L^{2}\right]}$ & $\left\|\mathbf{e}_{u}\right\|_{L^{2}\left[L^{2}\right]}$ & $\left\|\mathbf{e}_{u}\right\|_{L^{2}\left[H_{0}^{1}\right]}$ \\
\hline $1 / 4$ & $2.622886 \mathrm{e}-02$ & $3.641451 \mathrm{e}-02$ & $4.077321 \mathrm{e}-01$ & $3.830270 \mathrm{e}-02$ & $4.044421 \mathrm{e}-02$ & $4.622579 \mathrm{e}-01$ \\
$1 / 8$ & $7.468060 \mathrm{e}-03$ & $5.081297 \mathrm{e}-03$ & $1.121258 \mathrm{e}-01$ & $1.059918 \mathrm{e}-02$ & $6.311232 \mathrm{e}-03$ & $1.430898 \mathrm{e}-01$ \\
$1 / 16$ & $1.623642 \mathrm{e}-03$ & $3.494807 \mathrm{e}-04$ & $1.861618 \mathrm{e}-02$ & $2.281578 \mathrm{e}-03$ & $4.347941 \mathrm{e}-04$ & $2.339245 \mathrm{e}-02$ \\
$1 / 32$ & $3.921602 \mathrm{e}-04$ & $3.786972 \mathrm{e}-05$ & $3.994014 \mathrm{e}-03$ & $5.508430 \mathrm{e}-04$ & $4.536641 \mathrm{e}-05$ & $4.817865 \mathrm{e}-03$ \\
$1 / 64$ & $1.203874 \mathrm{e}-04$ & $4.631133 \mathrm{e}-06$ & $9.527884 \mathrm{e}-04$ & $1.447998 \mathrm{e}-04$ & $5.654464 \mathrm{e}-06$ & $1.129373 \mathrm{e}-03$ \\
$1 / 128$ & $4.277273 \mathrm{e}-05$ & $5.977846 \mathrm{e}-07$ & $2.350081 \mathrm{e}-04$ & $4.162113 \mathrm{e}-05$ & $7.911620 \mathrm{e}-07$ & $2.770349 \mathrm{e}-04$ \\
\hline Norm & 0.39043 & 0.82131 & 2.88478 & 0.54839 & 0.96586 & 3.39249 \\
\hline Rate & 1.89188 & 3.2279 & 2.1903 & 1.99603 & 3.1955 & 2.1931 \\
\hline
\end{tabular}

FiguRE 6.1. Rates of convergence for the $2 \mathrm{~d}$ solution with $k=1$.

\begin{tabular}{|c|ccc|ccc|}
\hline$h / 2=\tau$ & $\left\|e_{p}(1)\right\|_{L^{2}(\Omega)}$ & $\left\|\mathbf{e}_{u}(1)\right\|_{L^{2}(\Omega)}$ & $\left\|\mathbf{e}_{u}(1)\right\|_{H_{0}^{1}(\Omega)}$ & $\left\|e_{p}\right\|_{L^{2}\left[L^{2}\right]}$ & $\left\|\mathbf{e}_{u}\right\|_{L^{2}\left[L^{2}\right]}$ & $\left\|\mathbf{e}_{u}\right\|_{L^{2}\left[H_{0}^{1}\right]}$ \\
\hline $1 / 4$ & $4.167757 \mathrm{e}-02$ & $5.083148 \mathrm{e}-02$ & $5.501736 \mathrm{e}-01$ & $5.171707 \mathrm{e}-02$ & $4.665821 \mathrm{e}-02$ & $5.406396 \mathrm{e}-01$ \\
$1 / 8$ & $1.428320 \mathrm{e}-02$ & $1.168989 \mathrm{e}-02$ & $2.017905 \mathrm{e}-01$ & $1.724914 \mathrm{e}-02$ & $1.080494 \mathrm{e}-02$ & $2.010640 \mathrm{e}-01$ \\
$1 / 16$ & $2.537868 \mathrm{e}-03$ & $8.649203 \mathrm{e}-04$ & $3.503035 \mathrm{e}-02$ & $3.180688 \mathrm{e}-03$ & $7.939096 \mathrm{e}-04$ & $3.436079 \mathrm{e}-02$ \\
$1 / 32$ & $6.635174 \mathrm{e}-04$ & $8.712653 \mathrm{e}-05$ & $7.075149 \mathrm{e}-03$ & $7.470535 \mathrm{e}-04$ & $8.168104 \mathrm{e}-05$ & $7.004362 \mathrm{e}-03$ \\
$1 / 64$ & $1.444848 \mathrm{e}-04$ & $6.134461 \mathrm{e}-06$ & $1.138592 \mathrm{e}-03$ & $1.890673 \mathrm{e}-04$ & $9.815277 \mathrm{e}-06$ & $1.608875 \mathrm{e}-03$ \\
$1 / 128$ & $4.781144 \mathrm{e}-05$ & $9.808394 \mathrm{e}-07$ & $3.248874 \mathrm{e}-04$ & $5.121140 \mathrm{e}-05$ & $1.233694 \mathrm{e}-06$ & $3.832083 \mathrm{e}-04$ \\
\hline Norm & 0.39043 & 0.82131 & 2.88478 & 0.54839 & 0.96586 & 3.39249 \\
\hline Rate & 2.0187 & 3.2659 & 2.2384 & 2.04355 & 3.1322 & 2.1572 \\
\hline
\end{tabular}

Figure 6.2. Rates of convergence for the $2 \mathrm{~d}$ solution with $k=1$

(different mesh on each time interval).

\begin{tabular}{|c|ccc|ccc|}
\hline$h / 2=\tau$ & $\left\|e_{p}(1)\right\|_{L^{2}(\Omega)}$ & $\left\|\mathbf{e}_{u}(1)\right\|_{L^{2}(\Omega)}$ & $\left\|\mathbf{e}_{u}(1)\right\|_{H_{0}^{1}(\Omega)}$ & $\left\|e_{p}\right\|_{L^{2}\left[L^{2}\right]}$ & $\left\|\mathbf{e}_{u}\right\|_{L^{2}\left[L^{2}\right]}$ & $\left\|\mathbf{e}_{u}\right\|_{L^{2}\left[H_{0}^{1}\right]}$ \\
\hline $1 / 8$ & $7.760464 \mathrm{e}-03$ & $1.148147 \mathrm{e}-02$ & $2.216751 \mathrm{e}-01$ & $2.282557 \mathrm{e}-02$ & $2.205550 \mathrm{e}-02$ & $4.504727 \mathrm{e}-01$ \\
$1 / 12$ & $3.050692 \mathrm{e}-03$ & $2.167790 \mathrm{e}-03$ & $7.942503 \mathrm{e}-02$ & $8.138287 \mathrm{e}-03$ & $4.190167 \mathrm{e}-03$ & $1.556958 \mathrm{e}-01$ \\
$1 / 16$ & $1.756498 \mathrm{e}-03$ & $8.244456 \mathrm{e}-04$ & $4.219413 \mathrm{e}-02$ & $4.265093 \mathrm{e}-03$ & $1.500999 \mathrm{e}-03$ & $7.807455 \mathrm{e}-02$ \\
$1 / 20$ & $1.203159 \mathrm{e}-03$ & $4.073514 \mathrm{e}-04$ & $2.635061 \mathrm{e}-02$ & $2.670504 \mathrm{e}-03$ & $7.206254 \mathrm{e}-04$ & $4.704667 \mathrm{e}-02$ \\
$1 / 24$ & $9.074159 \mathrm{e}-04$ & $2.323340 \mathrm{e}-04$ & $1.806317 \mathrm{e}-02$ & $1.852352 \mathrm{e}-03$ & $4.060391 \mathrm{e}-04$ & $3.153842 \mathrm{e}-02$ \\
\hline Norm & 0.091174 & 0.618806 & 3.636961 & 0.271529 & 1.012584 & 5.951344 \\
\hline Rate & 1.9539 & 3.5342 & 2.2753 & 2.2844 & 3.6329 & 2.4202 \\
\hline
\end{tabular}

FiguRE 6.3. Rates of convergence for the $3 \mathrm{~d}$ solution with $k=1$.

\begin{tabular}{|c|c|ccc|ccc|}
\hline$h / 2$ & $\tau$ & $\left\|e_{p}(1)\right\|_{L^{2}(\Omega)}$ & $\left\|\mathbf{e}_{u}(1)\right\|_{L^{2}(\Omega)}$ & $\left\|\mathbf{e}_{u}(1)\right\|_{H_{0}^{1}(\Omega)}$ & $\left\|e_{p}\right\|_{L^{2}\left[L^{2}\right]}$ & $\left\|\mathbf{e}_{u}\right\|_{L^{2}\left[L^{2}\right]}$ & $\left\|\mathbf{e}_{u}\right\|_{L^{2}\left[H_{0}^{1}\right]}$ \\
\hline $1 / 16$ & $1 / 4$ & $1.623642 \mathrm{e}-03$ & $3.494801 \mathrm{e}-04$ & $1.861613 \mathrm{e}-02$ & $2.281143 \mathrm{e}-03$ & $4.346687 \mathrm{e}-04$ & $2.338486 \mathrm{e}-02$ \\
$1 / 25$ & $1 / 5$ & $6.472482 \mathrm{e}-04$ & $8.184200 \mathrm{e}-05$ & $6.785314 \mathrm{e}-03$ & $9.091463 \mathrm{e}-04$ & $9.900308 \mathrm{e}-05$ & $8.280692 \mathrm{e}-03$ \\
$1 / 36$ & $1 / 6$ & $3.091081 \mathrm{e}-04$ & $2.633494 \mathrm{e}-05$ & $3.116395 \mathrm{e}-03$ & $4.341717 \mathrm{e}-04$ & $3.143829 \mathrm{e}-05$ & $3.742657 \mathrm{e}-03$ \\
$1 / 49$ & $1 / 7$ & $1.661525 \mathrm{e}-04$ & $1.026424 \mathrm{e}-05$ & $1.644290 \mathrm{e}-03$ & $2.333757 \mathrm{e}-04$ & $1.217573 \mathrm{e}-05$ & $1.958177 \mathrm{e}-03$ \\
$1 / 64$ & $1 / 8$ & $9.719806 \mathrm{e}-05$ & $4.567068 \mathrm{e}-06$ & $9.526238 \mathrm{e}-04$ & $1.365229 \mathrm{e}-04$ & $5.399806 \mathrm{e}-06$ & $1.129298 \mathrm{e}-03$ \\
$1 / 81$ & $1 / 9$ & $6.061475 \mathrm{e}-05$ & $2.242196 \mathrm{e}-06$ & $5.908356 \mathrm{e}-04$ & $8.513853 \mathrm{e}-05$ & $2.646171 \mathrm{e}-06$ & $6.985738 \mathrm{e}-04$ \\
$1 / 100$ & $1 / 10$ & $3.974480 \mathrm{e}-05$ & $1.188276 \mathrm{e}-06$ & $3.861314 \mathrm{e}-04$ & $5.582491 \mathrm{e}-05$ & $1.400840 \mathrm{e}-06$ & $4.558145 \mathrm{e}-04$ \\
$1 / 121$ & $1 / 11$ & $2.713610 \mathrm{e}-05$ & $6.695778 \mathrm{e}-07$ & $2.630801 \mathrm{e}-04$ & $3.811493 \mathrm{e}-05$ & $7.888147 \mathrm{e}-07$ & $3.102413 \mathrm{e}-04$ \\
$1 / 144$ & $1 / 12$ & $1.915537 \mathrm{e}-05$ & $3.967973 \mathrm{e}-07$ & $1.854473 \mathrm{e}-04$ & $2.690532 \mathrm{e}-05$ & $4.672482 \mathrm{e}-07$ & $2.185437 \mathrm{e}-04$ \\
\hline Norm & & 0.39043 & 0.82131 & 2.88478 & 0.54839 & 0.96586 & 3.39249 \\
\hline Rate & wrt $h$ & 2.0173 & 3.0720 & 2.0844 & 2.0174 & 3.0940 & 2.1110 \\
\hline
\end{tabular}

FIGURE 6.4. Rates of convergence for the $2 \mathrm{~d}$ solution with $k=3$.

One of the more interesting conclusions of our analysis is that changing the mesh at every time step need not affect the rates of convergence if, for example, $h / \tau$ is bounded. More specifically, the rate of convergence for the velocity in 


\begin{tabular}{|c|c|ccc|ccc|}
\hline$h / 2$ & $\tau=(h / 2)^{3}$ & $\left\|e_{p}(1)\right\|_{L^{2}(\Omega)}$ & $\left\|\mathbf{e}_{u}(1)\right\|_{L^{2}(\Omega)}$ & $\left\|\mathbf{e}_{u}(1)\right\|_{H_{0}^{1}(\Omega)}$ & $\left\|e_{p}\right\|_{L^{2}\left[L^{2}\right]}$ & $\left\|\mathbf{e}_{u}\right\|_{L^{2}\left[L^{2}\right]}$ & $\left\|\mathbf{e}_{u}\right\|_{L^{2}\left[H_{0}^{1}\right]}$ \\
\hline $1 / 4$ & $1 / 64$ & $2.290879 \mathrm{e}-01$ & $3.898256 \mathrm{e}-02$ & $3.963173 \mathrm{e}-01$ & $1.966293 \mathrm{e}-01$ & $4.408524 \mathrm{e}-02$ & $4.914307 \mathrm{e}-01$ \\
$1 / 8$ & $1 / 512$ & $1.043136 \mathrm{e}-01$ & $7.317965 \mathrm{e}-03$ & $1.330243 \mathrm{e}-01$ & $9.565276 \mathrm{e}-02$ & $6.526791 \mathrm{e}-03$ & $1.317299 \mathrm{e}-01$ \\
$1 / 12$ & $1 / 1728$ & $1.204084 \mathrm{e}-01$ & $3.604188 \mathrm{e}-03$ & $5.861764 \mathrm{e}-02$ & $7.112988 \mathrm{e}-02$ & $3.289917 \mathrm{e}-03$ & $6.818552 \mathrm{e}-02$ \\
$1 / 16$ & $1 / 4096$ & $1.051564 \mathrm{e}-01$ & $1.506426 \mathrm{e}-03$ & $3.266569 \mathrm{e}-02$ & $5.754381 \mathrm{e}-02$ & $1.790446 \mathrm{e}-03$ & $4.071769 \mathrm{e}-02$ \\
$1 / 20$ & $1 / 8000$ & $4.890937 \mathrm{e}-02$ & $9.868940 \mathrm{e}-04$ & $2.462391 \mathrm{e}-02$ & $4.854666 \mathrm{e}-02$ & $1.036450 \mathrm{e}-03$ & $2.671513 \mathrm{e}-02$ \\
$1 / 24$ & $1 / 13824$ & $4.441340 \mathrm{e}-02$ & $7.091685 \mathrm{e}-04$ & $1.903895 \mathrm{e}-02$ & $4.201176 \mathrm{e}-02$ & $6.581414 \mathrm{e}-04$ & $1.867446 \mathrm{e}-02$ \\
\hline Norm & & 0.39043 & 0.82131 & 2.88478 & 0.54839 & 0.96586 & 3.39249 \\
\hline Rate & wrt $h$ & 0.83905 & 2.2519 & 1.7410 & 0.84623 & 2.2818 & 1.8045 \\
\hline
\end{tabular}

FiguRE 6.5. Rates of convergence for the $2 \mathrm{~d}$ solution with $k=0$ (different mesh on each time interval).

$L^{2}\left[0, T ; H^{1}(\Omega)\right]$ for the $Q_{2} / Q_{1}$ Taylor-Hood element is

$$
O\left(h^{2}+\tau^{k+1}+\min \left(h^{2} / \tau, h / \sqrt{\tau}\right) h^{2}\right) .
$$

The last term is only present when different meshes are used for each time step and will only dominate if $h^{2} / \tau$ is unbounded. To construct such examples, at each time step a random pair $\left(\theta_{x}, \theta_{y}\right) \in[0,1]^{2}$ is selected from the uniform distribution and the quadrilateral mesh with grid points

$$
\left(x_{i}, y_{j}\right)=\left(\left(1-\theta_{x}\right) i / N-\theta_{x} \cos (\pi(1+i / N) / 2),\left(1-\theta_{y}\right) j / N-\theta_{y} \cos (\pi(1+j / N) / 2)\right),
$$

is constructed on $[-1,1]^{2}$. Here $-N \leq i, j \leq N$ and $h \sim 2 / N$. The grid points in each direction are then a random homotopy of the uniform grid, $i / N$ and the Chebyshev points $-\cos (\pi(1+i / N) / 2)$.

To illustrate that the rates are unaffected when the underlying mesh changes from step to step and $\tau=h / 2$, Figure 6.2 shows the results for the same problem used for Figure 6.1 except that the mesh was changed every time step. While the errors are slightly greater in magnitude, the same optimal rates of convergence are observed.

To illustrate that the rates may deteriorate as $h^{2} / \tau \rightarrow \infty$, we present results obtained with $h \sim 2 / N$ and $\tau=1 / N^{3}$ and $k=0$ in Figure 6.5. With these parameters the rates would be the same as in the previous examples with a fixed mesh; when the mesh is changed at each time step Theorem 5.3 predicts reduction in the rate by $\min \left(h^{2} / \tau, h / \sqrt{\tau}\right)=h^{-1 / 2}$. While the rates in Figure 6.5 are lower, they are slightly better than those predicted by Theorem 5.3 .

\section{Appendix A. Bounding $\mathbf{u}_{h}$ In two Dimensions}

The sharper estimates available for the trilinear forms in two dimensions enable us to bound the approximate solutions in $L^{\infty}\left[0, T ; L^{2}(\Omega)\right]$.

Theorem A.1. Let $d=2$ and $\left(\mathbf{u}_{h}, p_{h}\right)$ be an approximate solution of the NavierStokes equations computed using the discontinuous Galerkin scheme (2.4) with $\mathbf{f} \in$ $L^{2}\left[0, T ; H^{-1}(\Omega)\right]$ and $\mathbf{u}_{0} \in W(\Omega)$. Then, there exists $C>0$ depending only on the domain and the constant $k$ such that

$$
\left\|\mathbf{u}_{h}\right\|_{L^{\infty}\left[0, T ; L^{2}(\Omega)\right]} \leq C\left(\left\|\mathbf{u}^{0}\right\|_{L^{2}(\Omega)}^{2}+\left(1 / \nu^{2}\right)\|\mathbf{f}\|_{L^{2}\left[0, T ; H^{-1}(\Omega)\right]}^{2}\right) .
$$

Proof. Let $\overline{\mathbf{u}}_{h}$ denote the exponential interpolant of $\mathbf{u}_{h}$ constructed in Definition 3.3. Since $\mathcal{Z}_{h}^{n}=\mathcal{P}_{k}\left[t^{n-1}, t^{n} ; Z_{h}^{n}\right]$ it follows that $\overline{\mathbf{u}}_{h} \in Z_{h}^{n}$ when $\mathbf{u}_{h} \in \mathcal{Z}_{h}^{n}$. Setting 
$\mathbf{v}_{h}=\overline{\mathbf{u}}_{h}$ into (2.4) the pressure term vanishes and we obtain

$$
\int_{t^{n-1}}^{t^{n}}\left(\left(\mathbf{u}_{h t}, \overline{\mathbf{u}}_{h}\right)+a\left(\mathbf{u}_{h}, \overline{\mathbf{u}}_{h}\right)+c\left(\mathbf{u}_{h}, \mathbf{u}_{h}, \overline{\mathbf{u}}_{h}\right)\right)+\left(\mathbf{u}_{+}^{n-1}-\mathbf{u}_{-}^{n-1}, \mathbf{u}_{+}^{n-1}\right)=\int_{t^{n-1}}^{t^{n}}\left\langle\mathbf{f}, \overline{\mathbf{u}}_{h}\right\rangle .
$$

Since $\mathbf{u}_{h t} \in \mathcal{P}_{k-1}\left[t^{n-1}, t^{n} ; U_{h}^{n}\right]$ the construction of $\overline{\mathbf{u}}_{h}$ gives

$$
\begin{aligned}
& \int_{t^{n-1}}^{t^{n}}\left(\mathbf{u}_{h t}, \overline{\mathbf{u}}_{h}\right)=\int_{t^{n-1}}^{t^{n}}\left(\mathbf{u}_{h t}, \mathbf{u}_{h}\right) e^{-\lambda\left(t-t^{n-1}\right)} \\
& =(1 / 2)\left\|\mathbf{u}_{-}^{n}\right\|_{L^{2}(\Omega)}^{2} e^{-\lambda\left(t^{n}-t^{n-1}\right)}-(1 / 2)\left\|\mathbf{u}_{+}^{n-1}\right\|^{2} \\
& \quad+(\lambda / 2) \int_{t^{n-1}}^{t^{n}}\left\|\mathbf{u}_{h}(t)\right\|_{L^{2}(\Omega)}^{2} e^{-\lambda\left(t-t^{n-1}\right)} .
\end{aligned}
$$

Upon recalling that $\left\|\nabla \overline{\mathbf{u}}_{h}\right\|_{L^{2}\left[t^{n-1}, t^{n} ; L^{2}(\Omega)\right]} \leq C\left\|\nabla \mathbf{u}_{h}\right\|_{L^{2}\left[t^{n-1}, t^{n} ; L^{2}(\Omega)\right]}$ it follows that

$$
\begin{aligned}
& (1 / 2)\left\|\mathbf{u}_{-}^{n}\right\|_{L^{2}(\Omega)}^{2} e^{-\lambda\left(t^{n}-t^{n-1}\right)}+(1 / 2)\left\|\left[\mathbf{u}_{h}^{n-1}\right]\right\|_{L^{2}(\Omega)}^{2} \\
& \quad+(\lambda / 2) \int_{t^{n-1}}^{t^{n}}\left\|\mathbf{u}_{h}\right\|_{L^{2}(\Omega)}^{2} e^{-\lambda\left(t-t^{n-1}\right)} \\
& =(1 / 2)\left\|\mathbf{u}_{-}^{n-1}\right\|_{L^{2}(\Omega)}^{2}+\int_{t^{n-1}}^{t^{n}}\left(\left\langle\mathbf{f}, \overline{\mathbf{u}}_{h}\right\rangle-a\left(\mathbf{u}_{h}, \overline{\mathbf{u}}_{h}\right)-c\left(\mathbf{u}_{h}, \mathbf{u}_{h}, \overline{\mathbf{u}}_{h}\right)\right) \\
& \leq(1 / 2)\left\|\mathbf{u}_{-}^{n-1}\right\|_{L^{2}(\Omega)}^{2}+\int_{t^{n-1}}^{t^{n}}\left((1 / 2 \nu)\|\mathbf{f}\|_{H^{-1}(\Omega)}^{2}+C \nu\left\|\nabla \mathbf{u}_{h}\right\|_{L^{2}(\Omega)}^{2}+\left|c\left(\mathbf{u}_{h}, \mathbf{u}_{h}, \overline{\mathbf{u}}_{h}\right)\right|\right)
\end{aligned}
$$

To bound the trilinear term first note that $c\left(\mathbf{u}_{h}, \mathbf{u}_{h}, \overline{\mathbf{u}}_{h}\right)=c\left(\mathbf{u}_{h}, \mathbf{u}_{h}, \overline{\mathbf{u}}_{h}-\mathbf{u}_{h}\right)$. Then, inequality (2.2) and Lemma 3.6 imply

$$
\begin{aligned}
& \int_{t^{n-1}}^{t^{n}}\left|c\left(\mathbf{u}_{h}, \mathbf{u}_{h}, \overline{\mathbf{u}}_{h}-\mathbf{u}_{h}\right)\right| \\
& \leq C \int_{t^{n-1}}^{t^{n}}\left\|\mathbf{u}_{h}\right\|_{L^{2}(\Omega)}^{1 / 2}\left\|\mathbf{u}_{h}\right\|_{H^{1}(\Omega)}^{1 / 2}\left(\left\|\mathbf{u}_{h}\right\|\left\|\overline{\mathbf{u}}_{h}-\mathbf{u}_{h}\right\|_{L^{2}(\Omega)}^{1 / 2}\left\|\overline{\mathbf{u}}_{h}-\mathbf{u}_{h}\right\|_{H^{1}(\Omega)}^{1 / 2}\right. \\
& \left.\quad+\left\|\overline{\mathbf{u}}_{h}-\mathbf{u}_{h}\right\|_{H^{1}(\Omega)}\left\|\mathbf{u}_{h}\right\|_{L^{2}(\Omega)}^{1 / 2}\left\|\mathbf{u}_{h}\right\|_{H^{1}(\Omega)}^{1 / 2}\right) \\
& \quad \leq\left\|_{h}\right\|_{L^{\infty}\left[t^{n-1}, t^{n} ; L^{2}(\Omega)\right]}^{1 / 2}\left\|\overline{\mathbf{u}}_{h}-\mathbf{u}_{h}\right\|_{L^{\infty}\left[t^{n-1}, t^{n} ; L^{2}(\Omega)\right]}^{1 / 2} \int_{t^{n-1}}^{t^{n}}\left\|\mathbf{u}_{h}\right\|_{H^{1}(\Omega)}^{3 / 2}\left\|\overline{\mathbf{u}}_{h}-\mathbf{u}_{h}\right\|_{H^{1}(\Omega)}^{1 / 2} \\
& \quad+C\left\|\mathbf{u}_{h}\right\|_{L^{\infty}\left[t^{n-1}, t^{n} ; L^{2}(\Omega)\right]} \int_{t^{n-1}}^{t^{n}}\left\|\overline{\mathbf{u}}_{h}-\mathbf{u}_{h}\right\|_{H^{1}(\Omega)}\left\|\mathbf{u}_{h}\right\|_{H^{1}(\Omega)} \\
& \leq C \lambda\left(t^{n}-t^{n-1}\right)\left\|\mathbf{u}_{h}\right\|_{L^{\infty}\left[t^{n-1}, t^{n} ; L^{2}(\Omega)\right]} \int_{t^{n-1}}^{t^{n}}\left\|\mathbf{u}_{h}\right\|_{H^{1}(\Omega)}^{2} .
\end{aligned}
$$

Substituting the above inequality into A.1), and setting $\lambda=1 /\left(t^{n}-t^{n-1}\right)$ it follows that

$$
\begin{aligned}
& \left\|\mathbf{u}_{-}^{n}\right\|_{L^{2}(\Omega)}^{2} e^{-\lambda\left(t^{n}-t^{n-1}\right)}+\left\|\left[\mathbf{u}_{h}^{n-1}\right]\right\|_{L^{2}(\Omega)}^{2} \\
& \quad+\int_{t^{n-1}}^{t^{n}}\left(e^{-1} /\left(t^{n}-t^{n-1}\right)\right)\left\|\mathbf{u}_{h}\right\|_{L^{2}(\Omega)}^{2} \leq\left\|\mathbf{u}_{-}^{n-1}\right\|_{L^{2}(\Omega)}^{2} \\
& \quad+C \int_{t^{n-1}}^{t^{n}}\left((1 / \nu)\|\mathbf{f}\|_{H^{-1}(\Omega)}^{2}+\nu\left\|\nabla \mathbf{u}_{h}\right\|_{L^{2}(\Omega)}^{2}+\left\|\mathbf{u}_{h}\right\|_{L^{\infty}\left[t^{n-1}, t^{n} ; L^{2}(\Omega)\right]}\left\|\mathbf{u}_{h}\right\|_{H^{1}(\Omega)}^{2}\right) .
\end{aligned}
$$


Using the Lemma 3.5 to bound

$$
\left\|\mathbf{u}_{h}\right\|_{L^{\infty}\left[t^{n-1}, t^{n} ; L^{2}(\Omega)\right]}^{2} \quad \text { by } \quad\left(C_{k} /\left(t^{n}-t^{n-1}\right)\right)\left\|\mathbf{u}_{h}\right\|_{L^{2}\left[t^{n-1}, t^{n} ; L^{2}(\Omega)\right]}^{2}
$$

gives

$$
\begin{aligned}
& \left\|\mathbf{u}_{-}^{n}\right\|_{L^{2}(\Omega)}^{2} e^{-\lambda\left(t^{n}-t^{n-1}\right)}+\left\|\left[\mathbf{u}_{h}^{n-1}\right]\right\|_{L^{2}(\Omega)}^{2}+\left(1 / C_{k}\right)\left\|\mathbf{u}_{h}\right\|_{L^{\infty}\left[t^{n-1}, t^{n} ; L^{2}(\Omega)\right]}^{2} \\
& \leq\left\|\mathbf{u}_{-}^{n-1}\right\|_{L^{2}(\Omega)}^{2}+C \int_{t^{n-1}}^{t^{n}}\left((1 / \nu)\|\mathbf{f}\|_{H^{-1}(\Omega)}^{2}+\nu\left\|\nabla \mathbf{u}_{h}\right\|_{L^{2}(\Omega)}^{2}\right) \\
& \quad+C(k)\left(\int_{t^{n-1}}^{t^{n}}\left\|\mathbf{u}_{h}\right\|_{H^{1}(\Omega)}^{2}\right)^{2} .
\end{aligned}
$$

Using Lemma 5.1 to bound the last term on the right completes the proof.

\section{Appendix B. The Stokes projection}

Estimates for the parabolic Stokes projection $\mathbb{P}_{h}$ used the properties of the stationary Stokes projection in an essential fashion. For completeness we sketch the proofs these properties stated in Lemma 4.4 and Corollary 4.5.

Proof of Lemma 4.4. Define $\mathbf{f} \in H^{-1}(\Omega)$ by $\mathbf{f}(\mathbf{v})=a(\mathbf{u}, \mathbf{v})$; that is, $\mathbf{f}=-\nu \Delta \mathbf{u}$. If $\mathbf{u} \in V(\Omega)$, then $(\mathbf{u}, 0)$ is the solution of the weak statement

$$
\begin{gathered}
a(\mathbf{u}, \mathbf{v})+b(\mathbf{v}, p)=\mathbf{f}(\mathbf{v}), \\
b(\mathbf{u}, q)=0
\end{gathered}
$$

for all $(\mathbf{v}, q) \in H_{0}^{1}(\Omega) \times L^{2}(\Omega) / \mathbb{R}$. If $\left(\mathbf{u}_{h}, p_{h}\right) \in U_{h} \times Q_{h}$ is the Galerkin approximation, then $\mathbf{u}_{h}=\Pi_{h} \mathbf{u}$, and classical finite element approximation theory [5, 17, 31] states

$$
\begin{aligned}
\| \mathbf{u}- & \mathbf{u}_{h}\left\|_{H^{1}(\Omega)}+(1 / \nu)\right\| 0-p_{h} \|_{L^{2}(\Omega)} \\
& \leq C\left(\inf _{\mathbf{v}_{h} \in U_{h}}\left\|\mathbf{u}-\mathbf{v}_{h}\right\|_{H^{1}(\Omega)}+(1 / \nu) \inf _{q_{h} \in Q_{h}}\left\|0-q_{h}\right\|_{L^{2}(\Omega)}\right) \\
& \leq C \inf _{\mathbf{v}_{h} \in U_{h}}\left\|\mathbf{u}-\mathbf{v}_{h}\right\|_{H^{1}(\Omega)} .
\end{aligned}
$$

Duality is used to bound the $L^{2}(\Omega)$ norm of the projection error. Let $(\mathbf{v}, r) \in$ $H_{0}^{1}(\Omega) \times L^{2}(\Omega) / \mathbb{R}$ satisfy

$$
\begin{aligned}
a(\mathbf{w}, \mathbf{v})+b(\mathbf{w}, r) & =\left(\mathbf{u}-\mathbf{u}_{h}, \mathbf{w}\right), \\
b(\mathbf{v}, s) & =0,
\end{aligned}
$$

for all $(\mathbf{w}, s) \in H_{0}^{1}(\Omega) \times L^{2}(\Omega) / \mathbb{R}$. Then

$$
\left\|\mathbf{u}-\mathbf{u}_{h}\right\|_{L^{2}(\Omega)}^{2}=a\left(\mathbf{u}-\mathbf{u}_{h}, \mathbf{v}\right)+b\left(\mathbf{u}-\mathbf{u}_{h}, r\right) .
$$

Since

$$
a\left(\mathbf{u}-\mathbf{u}_{h}, \mathbf{v}_{h}\right)=b\left(\mathbf{v}_{h}, p_{h}\right)=-b\left(\mathbf{v}-\mathbf{v}_{h}, p_{h}\right) \quad \mathbf{v}_{h} \in U_{h}
$$

and

$$
b\left(\mathbf{u}-\mathbf{u}_{h}, r\right)=-b\left(\mathbf{u}_{h}, r\right)=-b\left(\mathbf{u}_{h}, r-r_{h}\right)=b\left(\mathbf{u}-\mathbf{u}_{h}, r-r_{h}\right) \quad r_{h} \in Q_{h},
$$


it follows that

$$
\begin{aligned}
\| \mathbf{u}- & \mathbf{u}_{h} \|_{L^{2}(\Omega)}^{2}=a\left(\mathbf{u}-\mathbf{u}_{h}, \mathbf{v}-\mathbf{v}_{h}\right)-b\left(\mathbf{v}-\mathbf{v}_{h}, p_{h}\right)+b\left(\mathbf{u}-\mathbf{u}_{h}, r-r_{h}\right) \\
& \leq C\left(\left\|\mathbf{u}-\mathbf{u}_{h}\right\|_{H^{1}(\Omega)}+\left\|p_{h}\right\|_{L^{2}(\Omega)} / \nu\right)\left(\nu\left\|\mathbf{v}-\mathbf{v}_{h}\right\|_{H^{1}(\Omega)}+\left\|r-r_{h}\right\|_{L^{2}(\Omega)}\right) \\
& \leq C \inf _{w_{h} \in U_{h}}\left\|\mathbf{u}-\mathbf{w}_{h}\right\|_{H^{1}(\Omega)}\left(\nu\left\|\mathbf{v}-\mathbf{v}_{h}\right\|_{H^{1}(\Omega)}+\left\|r-r_{h}\right\|_{L^{2}(\Omega)}\right) .
\end{aligned}
$$

If $\Omega$ is sufficiently smooth to guarantee $H^{2}(\Omega) \times H^{1}(\Omega)$ regularity for the Stokes problem, the last term is dominated by $C\left\|\mathbf{u}-\mathbf{u}_{h}\right\|_{L^{2}(\Omega)} h$ and the proof follows.

Estimates for the jump terms were based on the statement of Corollary 4.5, which is proved next.

Proof of Corollary 4.5. If the mesh is quasi-uniform, inverse estimates establish optimal interpolation of $P_{n}$ on $H^{1}(\Omega)$ :

$$
\begin{aligned}
\left\|\mathbf{u}-P_{n} \mathbf{u}\right\|_{H^{1}(\Omega)} & \leq\left\|\mathbf{u}-\Pi_{n} \mathbf{u}\right\|_{H^{1}(\Omega)}+\left\|\Pi_{n} \mathbf{u}-P_{n} \mathbf{u}\right\|_{H^{1}(\Omega)} \\
& \leq\left\|\mathbf{u}-\Pi_{n} \mathbf{u}\right\|_{H^{1}(\Omega)}+(C / h)\left\|\Pi_{n} \mathbf{u}-P_{n} \mathbf{u}\right\|_{L^{2}(\Omega)} \\
& \leq\left\|\mathbf{u}-\Pi_{n} \mathbf{u}\right\|_{H^{1}(\Omega)}+(2 C / h)\left\|\Pi_{n} \mathbf{u}-\mathbf{u}\right\|_{L^{2}(\Omega)} \\
& \leq C \inf _{\mathbf{v}_{h} \in U_{h}^{n}}\left\|\mathbf{u}-\mathbf{v}_{h}\right\|_{H^{1}(\Omega)} .
\end{aligned}
$$

Thus, $\left\|P_{n} \mathbf{u}\right\|_{H^{1}(\Omega)} \leq C\|\mathbf{u}\|_{H^{1}(\Omega)}$ that we now use to estimate $\left\|P_{n}\left(I-P_{m}\right) \mathbf{u}\right\|_{H^{-1}(\Omega)}$ :

$$
\begin{aligned}
\left\|P_{n}\left(I-P_{m}\right) \mathbf{u}\right\|_{H^{-1}(\Omega)} & =\sup _{\mathbf{v} \in H^{1}(\Omega)} \frac{\left(\mathbf{u}-P_{m} \mathbf{u}, P_{n} \mathbf{v}\right)}{\|\mathbf{v}\|_{H^{1}(\Omega)}} \\
& =\sup _{\mathbf{v} \in H^{1}(\Omega)} \frac{\left(\mathbf{u}-P_{m} \mathbf{u}, P_{n} \mathbf{v}-\mathbf{w}_{h}\right)}{\|\mathbf{v}\|_{H^{1}(\Omega)}}, \quad \mathbf{w}_{h} \in Z_{h}^{m} \\
& \leq C \sup _{\mathbf{v}_{h} \in Z_{h}^{n}} \frac{\left(\mathbf{u}-P_{m} \mathbf{u}, \mathbf{v}_{h}-\mathbf{w}_{h}\right)}{\left\|\mathbf{v}_{h}\right\|_{H^{1}(\Omega)}} \\
& \leq C\left\|\mathbf{u}-P_{m} \mathbf{u}\right\|_{L^{2}(\Omega)} \inf _{\mathbf{w}_{h} \in Z_{h}^{m}} \sup _{\mathbf{v}_{h} \in Z_{h}^{n}} \frac{\left\|\mathbf{v}_{h}-\mathbf{w}_{h}\right\|_{L^{2}(\Omega)}}{\left\|\mathbf{v}_{h}\right\|_{H^{1}(\Omega)}} \\
& \leq C h \inf _{\mathbf{u}_{h} \in U_{h}^{m}}\left\|\mathbf{u}-\mathbf{u}_{h}\right\|_{L^{2}(\Omega)} \inf _{\mathbf{w}_{h} \in Z_{h}^{m}} \sup _{\mathbf{v}_{h} \in Z_{h}^{n}} \frac{\left\|\mathbf{v}_{h}-\mathbf{w}_{h}\right\|_{L^{2}(\Omega)}}{\left\|\mathbf{v}_{h}\right\|_{H^{1}(\Omega)}}
\end{aligned}
$$

Under the hypotheses of the lemma the last term is bounded by a constant of the form $C h$. To establish this, fix $\mathbf{v}_{h} \in Z_{h}^{n}$ and let $\mathbf{v}$ be the solution of the Stokes problem with right-hand side $\mathbf{f}(\mathbf{z})=a\left(\mathbf{v}_{h}, \mathbf{z}\right)$,

$$
a(\mathbf{v}, \mathbf{z})+b(\mathbf{z}, p)=a\left(\mathbf{v}_{h}, \mathbf{z}\right), \quad b(\mathbf{v}, q)=0, \quad(\mathbf{z}, q) \in H_{0}^{1}(\Omega) \times L^{2}(\Omega) / \mathbb{R} .
$$

Then $\|\mathbf{v}\|_{H^{1}(\Omega)} \leq\left\|\mathbf{v}_{h}\right\|_{H^{1}(\Omega)}$ and $\mathbf{v}_{h}=\Pi_{n} \mathbf{v}$. It follows that $\left\|\mathbf{v}-\mathbf{v}_{h}\right\|_{L^{2}(\Omega)} \leq$ $C h\left\|\mathbf{v}_{h}\right\|_{H^{1}(\Omega)}$. Then $\mathbf{w}_{h}=\Pi_{m} \mathbf{v}$ satisfies $\left\|\mathbf{v}-\mathbf{w}_{h}\right\|_{L^{2}(\Omega)} \leq C h\left\|\mathbf{w}_{h}\right\|_{H^{1}(\Omega)}$, and the triangle inequality, $\left\|\mathbf{v}_{h}-\mathbf{w}_{h}\right\|_{L^{2}(\Omega)} \leq\left\|\mathbf{v}-\mathbf{v}_{h}\right\|_{L^{2}(\Omega)}+\left\|\mathbf{v}-\mathbf{w}_{h}\right\|_{L^{2}(\Omega)}$ can then be used to show

$$
\left\|P_{n}\left(I-P_{m}\right) \mathbf{u}\right\|_{H^{-1}(\Omega)} \leq C h^{2} \inf _{\mathbf{u}_{h} \in U_{h}^{m}}\left\|\mathbf{u}-\mathbf{u}_{h}\right\|_{L^{2}(\Omega)} \mathbf{u} \in V(\Omega) .
$$




\section{REFERENCES}

[1] D. N. Arnold, F. Brezzi, B. Cockburn and D. L. Marini, Unified analysis of discontinuous Galerkin methods for elliptic problems, SIAM J. Numer. Anal. 39, 2001-02, pp 1749-1779. MR 1885715 (2002k:65183)

[2] G. Baker, V. Dougalis and O. Karakashian, On a higher order accurate fully discrete Galerkin approximation to the Navier-Stokes equations, Math. Comp. 39 1982, pp 339-375. MR669634 (84h:65096)

[3] R. E. BANK AND R.F. SAntos, Analysis of some moving space-time finite element methods, SIAM J. Numer. Anal. 30, 1993, pp 1-18 MR.1202654 (93m:65141)

[4] M. Bause, On optimal convergence rates for higher-order Navier-Stokes approximations I: Error estimates for spatial discretization, IMA J. Numer. Anal. 25 2005, pp 812-841. MR2170525 (2006f:76044)

[5] F. BrezzI, On the existence, uniqueness and approximation of saddle point problems arising from Lagrangian multipliers, RAIRO Anal. Numer. 8 1974, pp 129-151. MR0365287 $(51: 1540)$

[6] F. Brezzi And M. Fortin Mixed and Hybrid Finite Element Methods, Springer-Verlag, 1991. MR1115205 (92d:65187)

[7] K. Chrysafinos And N. J. Walkington, Error estimates for the discontinuous Galerkin methods for parabolic equations, SIAM J. Numer. Anal., 44 No1, 2006, pp 349-366. MR:2217386 (2007c:65075)

[8] K. Chrysafinos and N. J. Walkington, Error estimates for the discontinuous Galerkin methods for implicit parabolic equations, SIAM J. Numer. Anal., 43 No6, 2006, pp 24782499. MR2206444 (2006j:65280)

[9] K. Chrysafinos and Noel J. Walkington, Lagrangian and moving mesh finite element methods for the advection-diffusion equation, ESAIM $M^{2} A N, 42$ No1, 2008, pp 27-56. MR2387421

[10] P. G. Ciarlet, The finite element method for elliptic problems, SIAM, Classics In Applied Math, 2002. MR1930132

[11] B. Cockburn, G. E. Karnadiakis, and C. W. Shu, The development of discontinuous Galerkin methods, in Discontinuous Galerkin methods (Newport, RI, 1999), Springer, Berlin, 2000, pp 3-50. MR1842161(2002e:65002)

[12] P. Constantin and C. Foias, Navier-Stokes equations, Chicago Lectures in Mathematics, 1988. MR 972259 (90b:35190)

[13] M. Delfour, W. Hager And F. Trochu, Discontinuous Galerkin Methods for Ordinary Differential Equations, Math. Comp, 36, 1981, pp 455-473. MR606506 (82b:65066)

[14] T. F. Dupont and Yinguie Liu, Symmetric error estimates for moving mesh Galerkin methods for advection-diffusion equations, SIAM J. Numer. Anal. 40, 2002, pp 914-927. MR.1949398 (2004b:65147)

[15] K. ERIKSSON, C. Johnson And V. Thomée, Time discretization of parabolic problems by the discontinuous Galerkin method, RAIRO Modél. Math. Anal. Numér., 29, 1985, pp 611-643. MR826227(87e:65073)

[16] C. Ross Ethier and D. A. Steinman Exact fully 3D Navier-Stokes solutions for benchmarking IJNME, 19(5), July 2009, pp 369-375.

[17] V. Girault and P-A. Raviart, Finite element methods for Navier-Stokes equation, SpingerVerlag, Berlin, 1986. MR851383 (88b:65129)

[18] J. G. Heywood and R. Rannacher, Finite element approximation of the nonstationary Navier-Stokes problem. I. Regularity of solutions and second-order error estimates for spatial discretization, SIAM. J. Numer. Anal. 19 1982, pp 275-311. MR650052 (83d:65260)

[19] J. G. Heywood And R. Rannacher, Finite element approximation of the nonstationary Navier-Stokes problem, III. Smoothing property and higher order estimates for spatial discretization, SIAM. J. Numer. Anal. 25 1988, pp 489-512. MR.942204 (89k:65114)

[20] J. G. Heywood and R. Rannacher, Finite element approximation of the nonstationary Navier-Stokes problem. IV. Error analysis for second order discretization, SIAM J. Numer. Anal. 27 1990, pp. 355-384. MR.1043610 (92c:65133)

[21] L. S. Hou, Error estimates for semidiscrete finite element approximations of the Stokes equations under minimal regularity assumptions, J. Sci. Comput. 16, 2001, pp 287-317. MR.1873285(2002i:65103) 
[22] P. JAmet, Galerkin-type approximations which are discontinuous in time for parabolic equations in a variable domain, SIAM J. Numer. Anal. 15, 1978, pp 912-928. MR507554 (80e:65102)

[23] C. Johnson, Numerical Solution of Partial Differential Equations by the Finite Element Method, Cambridge, 1987. MR.911477 (89b:65003b)

[24] T. Katsaounis, Fully discrete nonconforming finite element schemes for the nonstationary Navier-Stokes equations, East-West J. Numer. Math., 6, 1998, pp 273-298. MR1667308 (2000b:76068)

[25] P. LASAint AND P.-A. Raviart, On a finite element method for solving the neutron transport equation, in Mathematical aspects of finite elements in partial differential equations, C. de Boor, ed., Academic Press, New York, 1974, pp. 89-123. MR0658142 (58:31918)

[26] P. L. Lions, Mathematical Topics in Fluid Mechanics, Volume 1: Incompressible Models, Oxford Press, Oxford, U.K., 1996. MR.1422251 (98b:76001)

[27] Yinguie Liu, R.E. Bank, T.F Dupont, S. Garcia and R. P. Santos, Symmetric error estimates for moving mesh mixed methods for advection-diffusion equations, SIAM J. Numer. Anal. 40, 2003, pp 2270-2291. MR 1974185 (2004f:65141)

[28] O. Pironeau, On the transport diffusion algorithm and its application to the Navier-Stokes equations, Numer. Math., 38, 1982, pp 309-332. MR654100 (83d:65258)

[29] Shapiro, A The use of an exact solution of the Navier-Stokes equations in a validation test of a three-dimensional non-hydrostatic numerical model, Mon. Wea. Rev., 121, 1993, pp 2420-2425.

[30] E. SüLI, Convergence and nonlinear stability of the Lagrange Galerkin method for the NavierStokes equations, Numer. Math., 53, 1998, pp. 457-483. MR951325(90b:65214)

[31] R. Temam, Navier-Stokes Equations, North Holland, 1979. MR603444 (82b:35133)

[32] R. Temam, Navier-Stokes equations and nonlinear functional analysis, Second Edition, CBNS-NSF Regional Conference Series in Applied Mathematics, SIAM, Philadelphia, 1995. MR1318914 (96e:35136)

[33] V. ThomÉE, Galerkin finite element methods for parabolic problems, Spinger-Verlag, Berlin, 1997 MR:1479170 (98m:65007)

[34] Wang, C. Y. Exact Solutions of the unsteady Navier-Stokes Equations, Appl. Mech. Rev. 42, 1989, pp. 269-282. MR1037589 (90k:76042)

[35] Wang, C. Y. Exact Solutions of the Steady-State Navier-Stokes Equations, Annual Rev. Fluid Mech. 23, 1991, pp. 159-177. MR1090331(92a:76030)

[36] N. J. Walkington, Convergence of the discontinuous Galerkin method for discontinuous solutions, SIAM J. Numer. Anal., 42, 2005, pp 1801-1817. MR2139223 (2006b:65147)

[37] N. J. Walkington, Compactness properties of the DG and CG time stepping schemes for parabolic equations, submitted to SINUM (June 2008), preprint available at http://www.math.cmu.edu/noelw.

Department of Mathematics, School of Applied Mathematics and Physical Sciences, National Technical University of Athens, Zografou Campus, Athens 15780, Greece

E-mail address: chrysafinos@math.ntua.gr

Department of Mathematical Sciences, Carnegie Mellon University, Pittsburgh, Pennsylvania 15213

E-mail address: noelw@andrew.cmu.edu 